\title{
Localized Proton Magnetic Resonance Spectroscopy of Mouse Brain In Vivo at High Magnetic Field Strength
}

\author{
Dissertation \\ for the award of the degree \\ "Doctor rerum naturalium" (Dr.rer.nat.) \\ of the Georg-August-Universität Göttingen \\ within the doctoral program ProPhys \\ of the Georg-August University School of Science (GAUSS) \\ submitted by \\ Alireza Abaei Tafresh \\ from Tehran
}

Göttingen, 2013 


\section{Betreuer}

Prof. Dr. Hans Hofsäss

Zweites Physikalisches Institut, Georg-August-Universität Göttingen

\section{Mitglieder der Prüfungskomission}

Referent: Prof. Dr. Hans Hofsäss

Zweites Physikalisches Institut, Georg-August-Universität Göttingen

Korreferent: PD Dr. Peter Dechent

MR-Forschung in der Neurologie und Psychatrie Universitätsmedizin Göttingen

\section{Weitere Mitglieder der Prüfungskomission}

\section{Prof. Dr. Astrid Pundt}

Institut für Materialphysik, Georg-August-Universität Göttingen

Prof. Dr. Joerg Enderlein

Drittes Physikalisches Institut, Georg-August-Universität Göttingen

Prof. Dr. Stephan Waack

Institut für Informatik, Fakultät für Mathematik und Informatik, Georg-August-Universität Göttingen

Prof. Dr. Carsten Damm

Institut für Informatik, Fakultät für Mathematik und Informatik, Georg-August-Universität Göttingen

Tag der mündlichen Prüfung: 13.05.2013 
1 Introduction

2 Physical Basics of Magnetic Resonance ......................................

2.1 Spin Magnetization .................................................................... 7

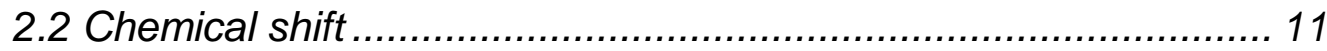

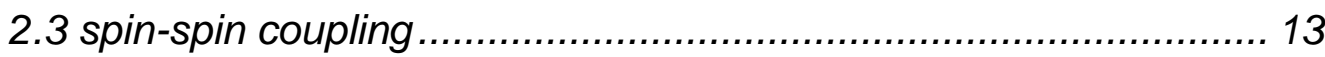

2.4 Single-Voxel MR Spectroscopy ….......................................... 15

3 Localized proton magnetic resonance spectroscopy at high magnetic field strength........................................................19

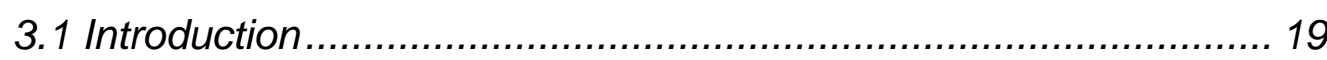

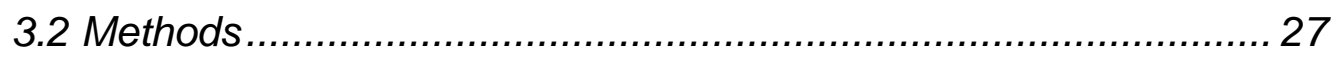

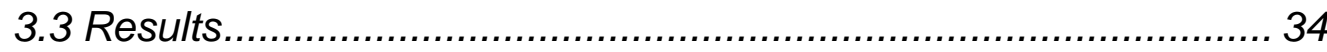

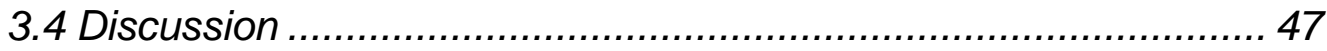

4 Regional metabolite concentrations of mouse brain in vivo...49

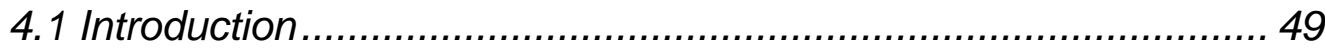

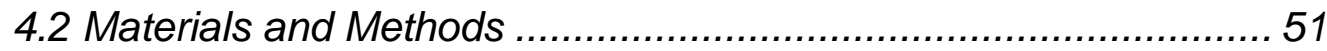

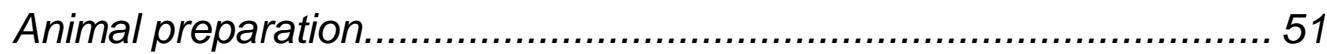

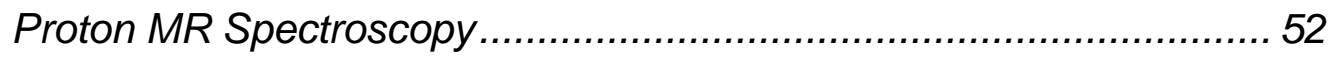

Quantification of metabolites ...................................................... 56

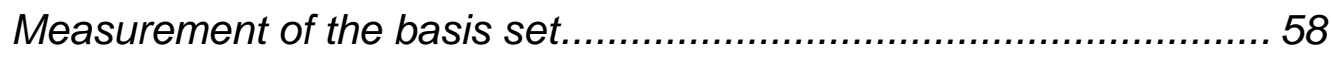

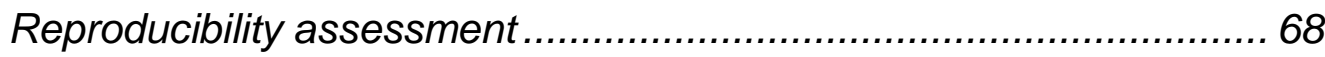

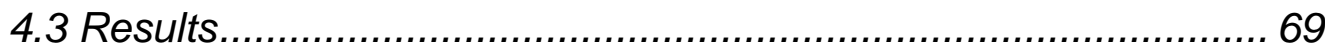

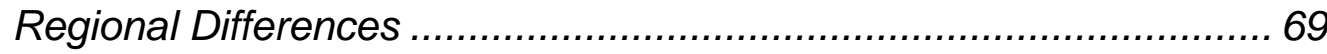

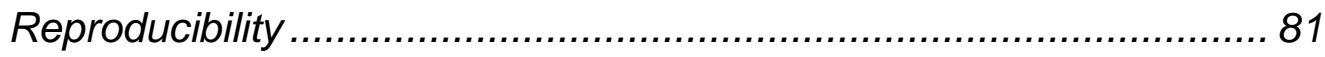

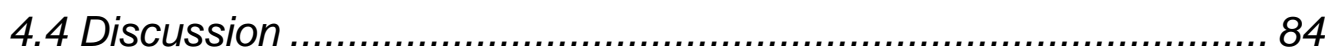

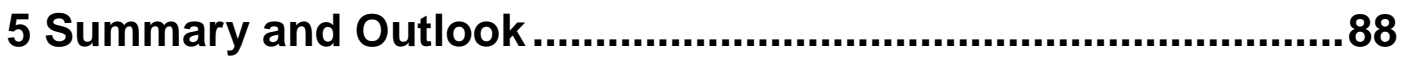

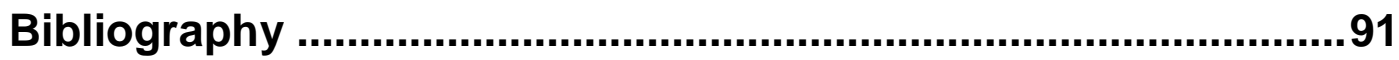

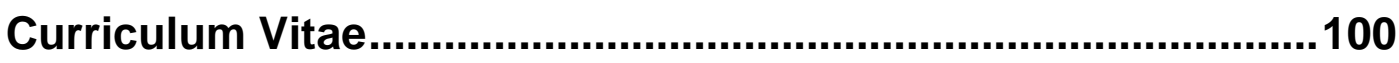

List of Publications .................................................................101 



\section{Chapter 1}

\section{Introduction}

Magnetic resonance spectroscopy (MRS) was established, upon independent discovery of nuclear magnetic resonance (NMR) phenomenon, by Bloch and Purcell in 1946 (Bloch, 1946, Bloch et al., 1946b, Bloch et al., 1946a, Purcell et al., 1946). They observed resonance absorption and emission of spins placed in a static, strong external magnetic field and described time evolution of the nuclear magnetization - in relation to the external magnetic fields and to the relaxation times $\left(T_{1}\right.$ and $\left.T_{2}\right)$. The method was initially of interest only to physicists for measuring the gyromagnetic ratios $(\gamma)$ of different elements in the periodic table. However, four years later, the chemical shift phenomenon came to light, in which influence of the chemical environment of a nucleus, on its resonance frequency, was realized (Dickinson, 1950, Proctor and Yu, 1950). Following the discovery of the spin echo (Hahn, 1950) and of spin-spin coupling (Ramsey and Purcell, 1952), NMR spectroscopy gradually developed into the most versatile technique for non-invasive probing of molecular structure, as well as molecular motions and reaction dynamics. The diagnostic value of (proton) NMR in medical applications was apprehended first by Damadian (Damadian, 1971), reporting the different magnetic relaxation times of malignant tumors from those of normal tissues. With the advent of linear magnetic field gradients (Lauterbur, 1973, Mansfield and 
Grannell, 1973), new opportunities were presented to derive spatial density information of nuclei inside an object that paved the way for technical developments of modern localized spectroscopy.

Complementary to the insights provided by structural and functional magnetic resonance imaging (MRI) methods, magnetic resonance spectroscopy (MRS) offers unique access to major tissue metabolites concentration in vivo. This particularly applies to proton MRS $\left({ }^{1} \mathrm{H}\right.$ MRS) studies of the central nervous system, where spectral recordings cover metabolites involved in energy metabolism, membrane turnover, glial proliferation, and neuroaxonal integrity. Accessible key metabolites are $\mathrm{N}$ acetylaspartate (NAA), a neuroaxonal marker linked to neuronal viability and function, creatine $(\mathrm{Cr})$ and phosphocreatine $(\mathrm{PCr})$ as important energy metabolites and constituents of all cells, choline-containing compounds (Cho) representing formation and degradation products of cell membranes such as glycerophosphocholine (GPC) and phosphocholine (PCh) which are predominantly present in oligodendrocytes, and myoinositol (Ins) as a marker for astrocytes. Alterations of these compounds have been demonstrated to be indicative for a variety of pathological processes and they are monitored during the course of disease progression. Other metabolites such as lactate (Lac), alanine (Ala), N-acetylaspartylglutamate (NAAG), aspartate (Asp), $\boldsymbol{V}$-aminobutyric acid (GABA), and taurine (Tau) can be used for a more detailed analysis of affected brain tissue. Specific marker compounds could be identified, through MRS, for glial cells (oligodendrocytes, astrocytes) and neurons (including their axons). As a consequence, technical developments of suitable localization sequences were rapidly followed by applications to human brain under both physiologic and pathologic conditions. STEAM (STimulated Echo Aquisition Mode)(Frahm et al., 1989a) and PRESS (Point RESolved Spectroscopy)(Bottomley, 1987) localization techniques are among those used for volume selection and have gained widespread acceptance. Pertinent studies cover a broad range of disorders, including focal brain lesions as well as neurometabolic and neurodegenerative diseases. Questions not only address diagnostic issues but expand to the (early) monitoring of therapeutic success. 
So far, a variety of localized spectroscopy studies were carried out on animals including mice (Schwarcz et al., 2003), rats (Gyngell et al., 1991a, Gyngell et al., 1991b, Gyngell et al., 1992, Fujimori et al., 1998, Michaelis et al., 1999, Wick et al., 1999, Liebetanz et al., 2003) and tree shrews (Czeh et al., 2001, Michaelis et al., 2001, van der Hart et al., 2002, Czeh et al., 2005). However, previous animal MRS studies were limited, in terms of spatial resolution and/or sensitivity, because of the use of relatively low field strength of $2.35 \mathrm{~T}$. Applications of MRS at high field is faced with several important hurdles that need to be surmounted, some of which involve signal loss due to Jmodulation of spin-coupled resonances, $T_{2}$ relaxation, chemical shift displacement error, increased magnetic susceptibility, residual eddy currents, and magnetic field instability (Frahm et al., 1989b, Howe et al., 1993, Di Costanzo et al., 2003). The recent availability of MR systems operating at higher magnetic fields, as well as the subsequent technical and methodological advances, have brought about substantial improvements in sensitivity and in spatial, temporal and spectral resolution, allowing reliable quantification of a much larger number of metabolites from smaller brain regions in reasonable measuring time (Gruetter et al., 1998).

Several years ago in vivo ${ }^{1} \mathrm{H}$ NMR spectroscopy at $9.4 \mathrm{~T}$ has first been demonstrated on the rat brain (Pfeuffer et al., 1999, Tkáć et al., 1999) and later on the mouse brain (Tkáć et al., 2004). However, there are several limitations in their method: (i) Very short echo time of 2 ms was selected for the acquisition of spectra. This may cause considerable contribution of the signal of unspecific macromolecules, which have very short $T_{2}$ relaxation times. This hampers the quality of the baseline of the spectra and, thus, increases the risk of under- and over-estimation in quantifying the concentration of metabolites. (ii) A surface coil was used, not only for signal reception but also for radiofrequency (RF) excitation. This may pose a risk of inhomogeneous excitation profile $\left(B_{1}\right)$, which has an influence on the quality of the localization of the volume-of-interest (VOI), in particular, when it is localized at distances far away from the surface coil. (iii) The instability of $B_{0}$ caused a noticeable frequency drift in the course of measurement, which required a correction for each spectrum, prior to averaging. (iv) An additional step was required in correction of spectra, possibly because application of 
very strong spoiler gradients, forced by the very short echo time, caused substantially uncompensated eddy currents. The spoiler gradients must be applied for spoiling the unwanted echo. (v) Seven RF pulses were used for the suppression of the water signal. This increases the risk of unwanted echo formation. In addition, magnetization exchange with water protons, as a result of long water suppression scheme, can attenuate methyl signal of creatine. (vi) Only orthogonal volumes-of-interest were used, which limit the localization for specific brain regions.

In the current work, centering on STEAM, the method of choice for single-voxel localization, these limitations will be overcome respectively: (i) optimal echo time (TE) of $10 \mathrm{~ms}$ will be employed, which minimizes the unspecific macromolecule signals while reducing substantial $T_{2}$ relaxation losses and J-modulation of coupled spins. (ii) Use of quadrature volume resonator will provide more homogeneous excitation profile. This will enable a sufficiently reliable localization to obtain adequate signals - even from the brain regions remote from the surface coil. (iii) state-of-the-art magnet and shim coils will ensure stability in $B_{0}$. (iv) $12 \mathrm{~cm}$ inner diameter self-shielded gradient coil insert (Resonance Research Inc, Billerica, MA, USA) will provide sophisticated eddy current compensation, while being capable of supplying up to $400 \mathrm{mTm}^{-1}$ in $80 \mu$ s rise time. (v) Use of only three RF pulses in water suppression scheme, in analogy to what is proposed by Ernst et al (Ernst and Hennig, 1995), will minimize the risk of unwanted echo formation. (vi) Use of oblique volume-of-interest will facilitate localization for specific brain regions.

With these improvements, the primary objective of the research presented in this thesis is to implement and optimize a STEAM localization technique on a 9.4 T MRI system, to perform state-of-the-art single-voxel ${ }^{1} \mathrm{H}$ MRS in vivo, taking full advantage of the gain in SNR and chemical shift dispersion at higher field strengths. Further, these modifications will require, (i) systematic investigation of bandwidths and inter-pulse delays of water suppression pulses for in vitro condition as well as for mouse brain in vivo, (ii) systematic investigation of the relative detectability of strongly coupled metabolite resonances at 9.4 T compared to lower field strength. 
The second objective of this project is concerned with the absolute quantification of regional neurochemical profiles in healthy mouse brain in vivo, to test and validate the developed spectroscopic method and the quantification technique. Development of in vivo techniques for characterizing the brains of mice is of great importance because a growing number of mutant mice are being generated for a better understanding of pathological mechanisms, which underlie human brain disorders. The non-invasiveness of ${ }^{1} \mathrm{H}$ MRS enables repeated assessment of longitudinal treatment on behaving mice, which represents a model of chronic human disorders. In this regard, high reproducibility of the method is essential to unveil subtle variation of neurochemical profile in the longitudinal investigations of physiological or pathophysiological processes. So far, there are only a few laboratories that can perform proton MRS studies of the mouse brain in vivo, probably because it has specific requirements (Lei et al., 2010, Öz et al., 2010, Oberg et al., 2008). Immobilization of mice is challenging because anesthetics may alter cerebral metabolism. The small size of the sample is another challenge because signal must be collected from much smaller subregions of the mouse brain, compared to that of human. In the previous work, using the smallest volume-of-interest (VOI) for mouse brain in vivo, metabolite concentrations were determined for only four different regions (Tkáć et al., 2004). Further, scyllo-Ins has not yet been described for mice in vivo, despite its detectable concentration in human brain (Michaelis et al., 1993a, Seaquist and Gruetter, 1998). Only one study has investigated the intra- and inter-individual variability in mouse brain in vivo (Öz et al., 2010).

Thus, to achieve the second objective of this project, (i) experimental setup, e.g., the selection of the coils, the method of anesthesia, the maintenance of body temperature, and the fixation of the head of the mouse, will be developed for repeated MRS of the same mouse and for examining the reproducibility of the method, (ii) VOI localization technique for MRS will be optimized for 10 different brain regions of anesthetized mice, (iii) $T_{1}$ and $T_{2}$ relaxation times from different brain regions of anesthetized mice will be measured for a correct quantification of metabolite concentrations, (iv) intra- and inter-individual reproducibility of MR spectroscopic acquisition protocol and of quantified data analysis will be assessed, ( $v$ ) absolute 
concentrations of 16 different brain metabolites, including scyllo-Ins from anesthetized mice, will be quantified and presented with necessary statistical values. Once established, the new high-field MRS protocol will be ready to be exploited for future studies on metabolic and cellular characterization of the brain in a large number of existing animal models. This will enable us to assess the metabolic profiles in genetically modified mice, including models of human brain in basic neuroscience studies. In fact, the experimental setup, which has been developed and established in the present work, recently provided a new insight into cerebral metabolism (Michaelis et al., 2009, Boretius et al., 2013).

In this thesis, after the physical basis of ${ }^{1} \mathrm{H}$ MRS is described (Chapter 2), optimization of STEAM sequence parameters as well as attributed modules is presented in Chapter 3. Firstly, the issues of water suppression, outer-volume suppression and automatic localized shimming techniques are addressed because they are stringent requirements for short-TE ${ }^{1} \mathrm{H}$ NMR spectroscopy that needs to be complied with. Further, relative detectability of strongly coupled metabolites at low-field (2.35 T) strength is compared to that at high-field (9.4 T) to further clarify the relative merits of field strengths. In Chapter 4, the experimental development for MRS of the brain of anesthetized mice is described. The acquisition of metabolite model spectra and the generation of the basis set, as a priori knowledge, are essential for the employed quantification technique, which enables a user-independent analysis. Special emphasis is placed on the characterization of potential impacts of signal loss, associated with $T_{1}$ and $\mathrm{T}_{2}$ relaxation, as well as of cerebrospinal fluid (CSF) contribution on metabolite quantification. Finally, the reliability and reproducibility of the in vivo metabolite concentration measurements are assessed. 


\section{Chapter 2}

\section{Physical Basics of Magnetic Resonance}

\subsection{Spin Magnetization}

Particular nuclei hold a property that is termed spin. Any atomic nucleus with an odd number of neutrons and/or protons, possesses angular momentum $\mathbf{L}$ and $a$ magnetic dipole moment, $\boldsymbol{\mu}$ which they are related according to:

$$
\boldsymbol{\mu}=\gamma \boldsymbol{L}=\gamma \hbar \boldsymbol{I}
$$

Where $\gamma$ is called gyromagnetic ratio, an intrinsic constant the particular nucleus that is determined by the ratio of the nuclear charge to its mass, I is the nuclear spin quantum number, and $\hbar$ is Planck's constant over $2 \pi$. The magnetic parameters of some selected nuclei commonly used in clinical MRS are enumerated in Table 2.1. 
Table 2.1 Basic properties of some popular nuclei in in vivo NMR

\begin{tabular}{lll}
\hline Nucleus & Spin & $\begin{array}{l}\boldsymbol{\gamma} / \mathbf{2} \boldsymbol{\pi} \\
\left(\mathbf{M H z ~ T}^{-1}\right)\end{array}$ \\
\hline${ }^{1} \mathrm{H}$ & $\frac{1}{2}$ & 42.58 \\
${ }^{13} \mathrm{C}$ & $\frac{1}{2}$ & 10.71 \\
${ }^{19} \mathrm{~F}$ & $\frac{1}{2}$ & 40.05 \\
${ }^{23} \mathrm{Na}$ & $\frac{3}{2}$ & 11.26 \\
${ }^{31} \mathrm{P}$ & $\frac{1}{2}$ & 17.23 \\
\hline \hline
\end{tabular}

Reproduced from (Storey, 2005)

In the presence of a static magnetic field $\left(B_{0}\right)$, there are $(2 I+1)$ possible spin states with integer steps ranging from $+I$ to $-I$, which is known as the Zeeman splitting. As an example, ${ }^{1} \mathrm{H}$ nucleus with $I=\frac{1}{2}$ can take two possible spin states of $+\frac{1}{2}$ and $-\frac{1}{2}$ corresponding to the parallel or anti-parallel alignment of nuclear magnetic moments with respect to the external magnetic field. It should be noted that larger magnetic fields give rise to greater alignment of the spins.

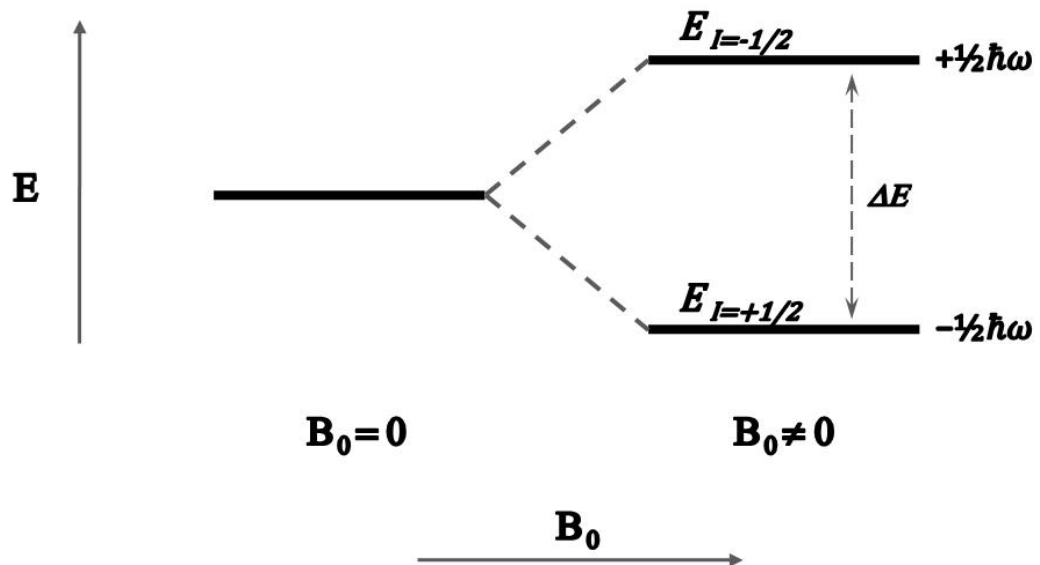

Figure 2.1: Zeeman splitting of nuclear spin states. In the absence of an external magnetic field $\left(B_{0}=0\right)$, the two energy eigenstates of the spin $1 / 2$ particle are degenerated. For $B_{0} \neq 0$, Zeeman splitting is observed. Reproduced from (Pohmann, 2011) 
The anti-parallel aligned level has a higher energy $\left(E_{I=-\frac{1}{2}}\right)$ than the parallel level $\left(E_{I=+\frac{1}{2}}\right)$. In this case, energy difference between two different energy eigenstates of $\pm 1 / 2 \gamma \hbar B_{0}$ will be (see Fig. 2.1)

$$
\Delta E=\gamma \hbar B_{0}
$$

Therefore, the Larmor frequency, $\omega_{0}$, which is the angular frequency at which the angular momentum precesses about the magnetic field axis, can be expressed as:

$$
\omega_{0}=\gamma B_{0}
$$

The population difference between the spin states, under thermal equilibrium conditions, is given by the Boltzmann's distribution Law:

$$
\frac{N_{\text {High }}}{N_{\text {Low }}}=e^{-\frac{\Delta E}{k_{B} T}}=e^{-\frac{\gamma \hbar B_{0}}{k_{B} T}}
$$

Where $\Delta E$ is known as the energy difference between adjacent Zeeman levels, $\mathrm{k}_{\mathrm{B}}$ is the Boltzmann's constant $\left(\mathrm{k}_{\mathrm{B}}=1.38 \cdot 10^{-23} \mathrm{JK} K^{-1}\right)$ and $T$ is the (absolute) temperature.

Given that the difference between energy state for protons at room temperature will be small for relatively small $B_{0}, \gamma \hbar B_{0} / k_{B} T \ll 1$, it is reasonable to make a firstorder approximation of $e^{x} \approx 1+x$.

Thus,

$$
\frac{N_{\text {High }}}{N_{\text {Low }}}=1-\frac{\gamma \hbar B_{0}}{k_{B} T}
$$

NMR signal intensity is dependent on the excess in population of nuclear spin in the lower energy state which is extremely small (e.g., $\left(N_{\text {Low }}-N_{\text {High }}\right) / N_{\text {Low }} \approx$ $\frac{\gamma \hbar B_{0}}{k_{B} T} \sim 10^{-5}$ for 1 Tesla). This is translated into an intrinsically low sensitivity of magnetic resonance techniques. The equilibrium magnetization $M_{0}$ is given by:

$$
\boldsymbol{M}_{\mathbf{0}}=\left(N_{\text {Low }}-N_{\text {High }}\right) \boldsymbol{\mu}
$$


The net magnetization in a system of nuclei with a spin $I$ in an external field $B_{0}$ and gyromagnetic ratio $\gamma$ can be described by the following equation:

$$
M_{0}=\frac{N_{s} \gamma^{2} \hbar^{2} I(I+1)}{3 k_{B} T} B_{0}
$$

Where $N_{S}$ is the total number of spins in the sample. Hence, higher static magnetic fields give rise to increased equilibrium magnetization, which is, in fact, an enhancement of the MR signal and sensitivity. 


\subsection{Chemical shift}

The underlying concept of NMR spectroscopy is based on the chemical shift phenomenon. Nuclei in different molecular environments precess at slightly different frequencies. This is a consequence of the fact that the rotation of the electron cloud around the nucleus induces diamagnetic shielding that opposes the external field $B_{0}$. Thus, the effective magnetic field experienced by the nucleus can be expressed as:

$$
B=B_{0}(1-\sigma)
$$

where $\sigma$ is a shielding (or screening) constant. Chemical shift is normally expressed in the dimensionless unit of parts per million (ppm) instead of in $\mathrm{Hz}$, which makes it independent of external magnetic field strength. It can be defined as:

$$
\delta_{p p m}=\frac{\omega_{i}-\omega_{r}}{\omega_{r}} \times 10^{6}
$$

where $\omega_{i}$ is the resonance frequency of the given nuclei and $\omega_{r}$ is an arbitrary chosen reference frequency. TMS [Tetramethylsilane, $\mathrm{Si}(\mathrm{CH} 3) 4$ ] and DSS (sodium 2,2dimethyl-2-silapentane-5-sulphonate) are commonly used substances as internal chemical shift reference $\left(\delta_{p p m}=0\right)$, in in vitro ${ }^{1} \mathrm{H}$ and ${ }^{13} \mathrm{C}$ spectroscopy. In in vivo situation, methyl singlet of $\mathrm{N}$-acetyl aspartate (NAA) with a chemical shift value of 2.01 ppm is often used as an in vivo standard in ${ }^{1} \mathrm{H}$ NMR spectrum. 


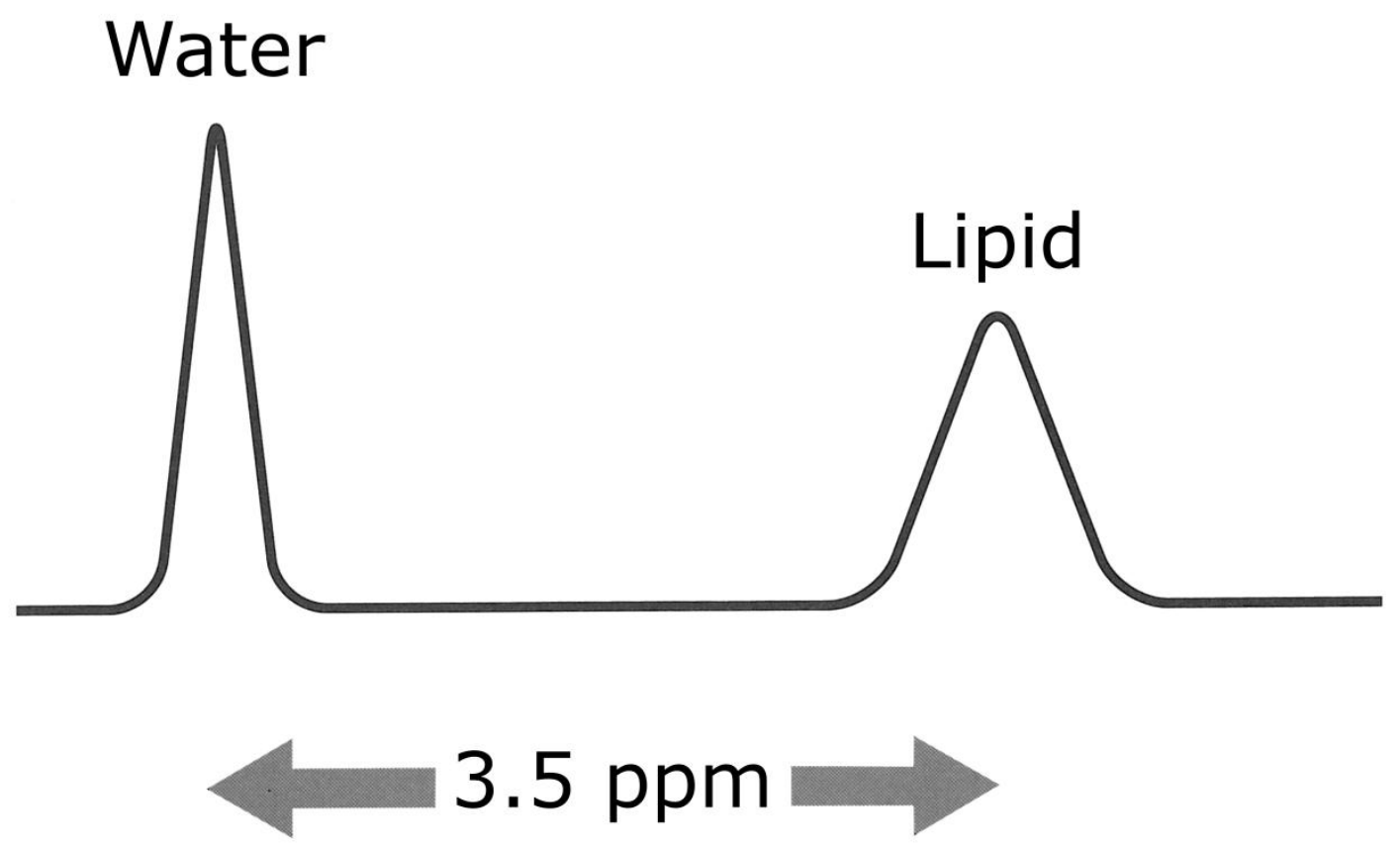

Figure 2.2: ${ }^{1} \mathrm{H}$ NMR spectrum acquired from a sample consisting of the fat and water. Reproduced from (de Graaf, 2007)

As an example, protons in the fat tissue experience stronger shielding than those in water. Therefore their resonance frequency is slightly lower, leading to approximately $3.5 \mathrm{ppm}$ shift of the spectral line to the right in the spectrum as shown in Figure 2.2. To this end, different metabolites can be identified on account of the fact that chemical shift values for given nuclei are different in each chemical environment. 


\section{3 spin-spin coupling}

Along with the chemical shift, the resonance frequency of a nucleus can be altered through another molecular interaction known as spin-spin coupling or J-coupling. This phenomenon results in the fine structure of the NMR resonances in the forms of splittings (or multiplets). The spin of one nucleus perturbs the energy levels of neighboring magnetic nuclei, through polarization of the bonding electrons within a molecule. The effective magnetic field sensed by one nucleus is dependent on the spin state of a vicinal coupled nucleus.

The frequency difference between the multiplet peaks reflects the J-coupling constant. This constant, which is measured in $\mathrm{Hz}$, implies intensity of coupling. In contrast to the chemical shift, J- coupling constant is independent of static magnetic field strength. The spin-spin coupling can be found for both heteronuclear (e.g., ${ }^{1} \mathrm{H}-{ }^{13} \mathrm{C}$ ) and homonuclear (e.g., ${ }^{1} \mathrm{H}-{ }^{1} \mathrm{H}$ ) interactions. Accordingly, the coupling constants observed for directly coupled spins (heteronuclear) are in the range of $100-200 \mathrm{~Hz}$, while those from the indirect couple spins are in the range of 1-15 $\mathrm{Hz}$ [refrence from $\mathrm{H} 3$ ]. The number of lines that appeared in the multiplet is $2 n I+1$ where $n$ is the number of equivalent coupled nuclei and $I$ their nuclear spin.

Considering the magnitude of J-coupling constants, with respect to the value of chemical shift distinction observed between the spins, coupling can be categorized into weak $(\mathrm{J} \ll \delta)$ or strong $(\mathrm{J} \approx \delta)$. For weak coupling, spectral analysis can be treated by first-order approximation; however, quantum-mechanical treatment is required for a strong coupling regime.

By convention, in the terminology used to describe spin systems, each individual spin will be assigned to letters, where alphabetical proximity of the letters indicating the coupling strength. e.g. $A B$ is a strongly coupled spin system whereas $A X$ is a weakly coupled one. The same hold true for systems with more than two spins, such as AX3 (e.g. lactate) and AMNPQ (e.g. glutamate). 


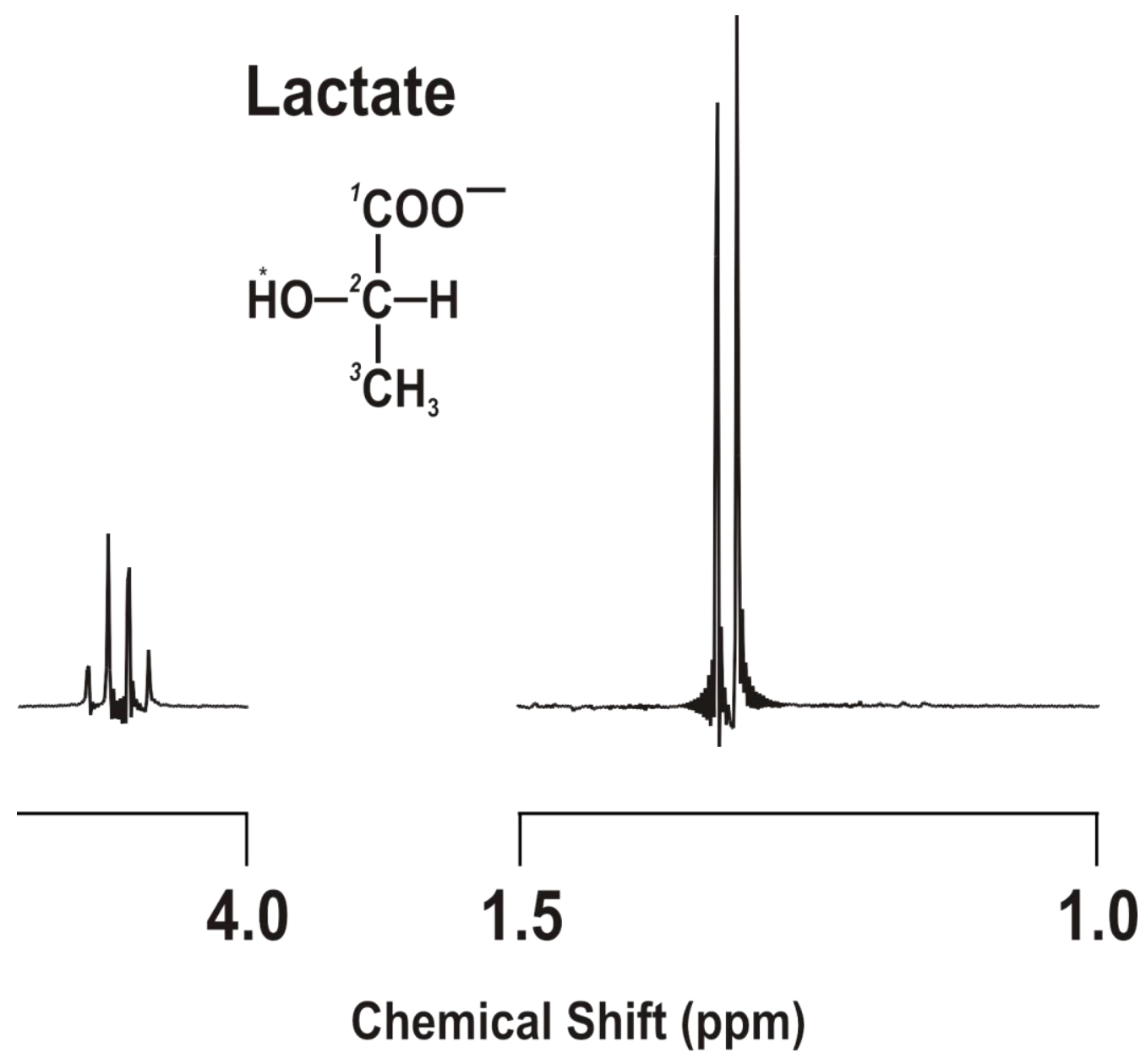

Figure 2.3: Molecular structure and ${ }^{1} \mathrm{H}$ spectrum of lactate. The experimental Localized ${ }^{1} \mathrm{H}$ MRS $1 D$ STEAM spectrum for a $50 \mathrm{mM}$ Lac phantom ( $27 \mu \mathrm{L}$ volume) at $9.4 \mathrm{~T}(\mathrm{TR} / \mathrm{TE}=15000 / 10 \mathrm{~ms}, 32$ scans) shows a doublet due to the $\mathrm{CH}_{3}$ group at $1.33 \mathrm{ppm}$, and a quartet from the $\mathrm{CH}$ group at $4.10 \mathrm{ppm}$. The "sinc wiggles" seen around the base of the peak are originated from truncation of data before FID has decayed to noise level, whereas the $T_{2}$ values of metabolites in vitro are likely to be longer than those in in vivo condition.

${ }^{1} \mathrm{H}$ spectrum of lactate, as shown in Figure 2.3 , is a typical example for a "weakly coupled system" and can be used to explain spin coupling. The resonance caused by methyl protons in the lactate molecule ( $X$ in the AX3 system) produces a doublet centered at $1.33 \mathrm{ppm}$ due to interactions with the methine proton (A), which holds two possible states of up or down. 
On the other hand, the methine $(\mathrm{CH})$ resonance at $4.10 \mathrm{ppm}$ is split into a quartet because of the coupling with three equivalent protons of the methyl group $\left(\mathrm{CH}_{3}\right)$ since, for each of which, four states are assumed.

\subsection{Single-Voxel MR Spectroscopy}

The simplest setup for NMR experiment can be established by applying an excitation RF pulse followed by detecting the induced oscillating current, arising from the rotating magnetic moments in an RF coil. The resultant signal, often called free induction decay (FID), is an exponentially damped sine wave. Fourier transform of the FID produces the NMR spectrum, providing the information on constituent nuclei of the sample.

In in vivo ${ }^{1} \mathrm{H}$ NMR spectroscopy, it is essential that information be obtained only from certain regions of the tissue. The advent and availability of static field gradients led to the development of numerous methods for in vivo localized MR spectroscopy.

STEAM (STimulated Echo Aquisition Mode (Frahm et al., 1987, Frahm et al., 1989a) spectroscopy and PRESS (Point RESolved Spectroscopy, (Bottomley, 1987)) localization technique, also known as double spin-echo, are among those used for volume selection and have gained widespread acceptance.

Both methods provide single-shot capabilities to acquire spectroscopic data from the VOI and are particularly advantageous over multi-shot techniques like image selected in vivo spectroscopy (ISIS), which is inherently based on multiple excitation followed by phase cycling scheme (Ordidge et al., 1986). This makes ISIS vulnerable to motion and contaminated with unwanted signal outside the VOI (Frahm and Hänicke, 2000). An additional advantage of single-shot is the ability to perform localized shimming, water suppression, and RF pulse gain adjustments on the desired VOI.

Both techniques employ three consecutive frequency-selective RF pulses in the presence of mutually orthogonal magnetic field gradients to achieve three-dimensional 
localization (volume selection). In this way, the desired VOI can be defined by the intersection of three perpendicular slices.

While STEAM uses three $90^{\circ}$ slice-selective excitation pulses, in PRESS the second and the third ones are replaced by two $180^{\circ}$ slice-selective refocusing RF pulses. In comparison with STEAM, application of $180^{\circ}$ pulses increases RF power requirements and results in a higher amount of power deposition. This problem becomes even more pronounced at higher field strength. Moreover, inferior voxel definition is assumed due to sensitivity to pulse imperfections ( $B_{1}$ inhomogeneity, non-ideal pulse shapes, offresonance effects, phase-shift errors)(Hore, 1983). This necessitates the use of pairs of spoiler gradients around the refocusing pulses to eliminate inadvertently generated transverse magnetization (Keevil, 2006), making the minimum attainable TE times longer and, consequently, resulting in more signal losses through T2 mechanisms compared to STEAM (Gillies and Morse, 2005). In addition, pulse imperfections give rise to a far more complicated evolution behaviour, which was investigated analytically, simulated numerically, and discussed in detail in previous publications (Trabesinger et al., 2005, Lange et al., 2006).

Figure 2.4 shows the STEAM pulse sequence, which will be used in this work. Application of three $90^{\circ}$ degree RF pulses initiates three FIDs (following each pulse), four normal spin-echoes, and one special signal generated after the third pulse, which is referred to as a 'stimulated echo' (Hahn, 1950). Spoiler gradients are required to preserve the desired signal, while eliminating the unwanted ones. 


\section{PRESS}

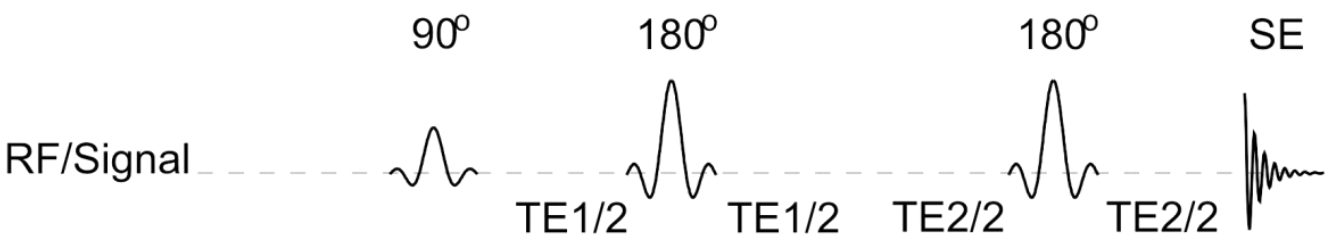

Slice $X$

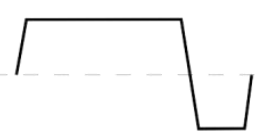

Slice $Y$

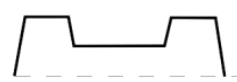

Slice Z

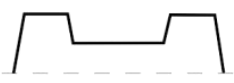

\section{STEAM}

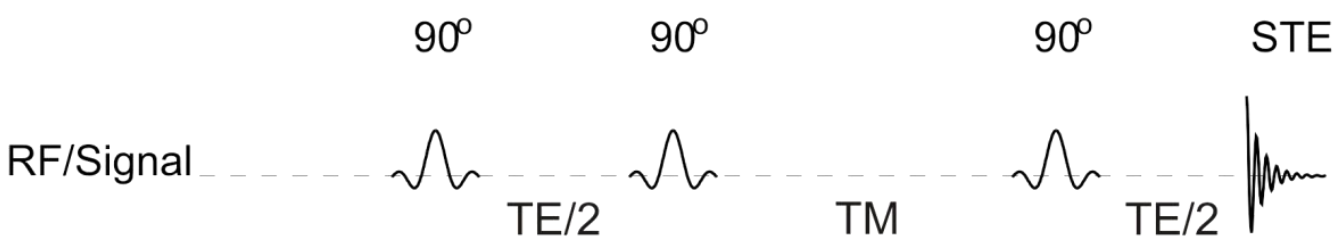

Slice $X$
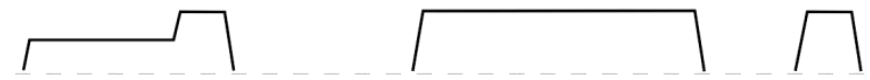

Slice $Y$
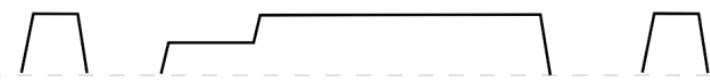

Slice Z
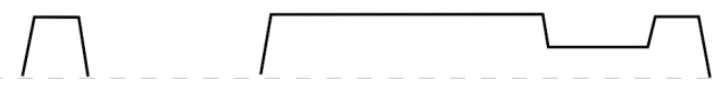

Figure 2.4: Schematic diagrams of the RF pulse and magnetic field gradients sequences commonly applied for localized Single-Voxel MRS in vivo. PRESS (top) and STEAM (bottom) sequence. 
The amplitude of the resultant signal in STEAM is theoretically half of that in its spin-echo counterparts PRESS, as a result of isotropic spin distribution (de Graaf, 2007).

However, to preclude aforementioned limitations, STEAM was considered the method of choice in this thesis, since it offers advantages for observation of very short $T_{2}$ metabolites with significant J-coupling modulation. 


\section{Chapter 3}

\section{Localized proton magnetic resonance spectroscopy at high magnetic field strength}

\subsection{Introduction}

\subsubsection{Localized shimming}

Placing the object inside the magnet will disturb the static magnetic field homogeneity. In localized magnetic resonance spectroscopy, this results in a variation of the Larmor frequency inside the volume of interest and thus, leads to a poor localization. In addition, magnetic field inhomogeneities will cause a widening of the resonance peaks of metabolites, a distortion of spectral lineshapes and thus, a reduction in the signal-to-noise ratio (SNR), which potentially reduces the accuracy of the metabolite quantitation. Another adverse outcome is spectral overlap, which has a profound effect on quantification accuracy of metabolite concentration (Stanley et al., 1995, Gruber et al., 2003, Macrì et al., 2004, Bartha, 2007). Moreover, macroscopic field heterogeneity causes deterioration in water (or fat) suppression quality, particularly when selective saturation method must be applied. Therefore, the improvement in magnetic field homogeneity is vital for spectral resolution and hence, for reliable and reproducible quantification of metabolites. 
Fast Automated Shimming Technique by Mapping Along Projections (FASTMAP) (Gruetter and Boesch, 1992, Gruetter, 1993, Gruetter and Tkáć, 2000) is a widely used localized automated shimming approach, which rapidly acquires information from six linear field map projections to adjust all first- $(X, Y, Z)$ and second-order (Z2, ZX, ZY, X2Y2, 2XY) shim coils (see Fig. 3 .1)(Faber and Webb, 2007, Koch et al., 2009). In other words, the required correction current changes for the second-order spherical harmonic shim fields are calculated through decomposition of magnetic field inhomogeneities into first- and second-order spherical harmonic functions.
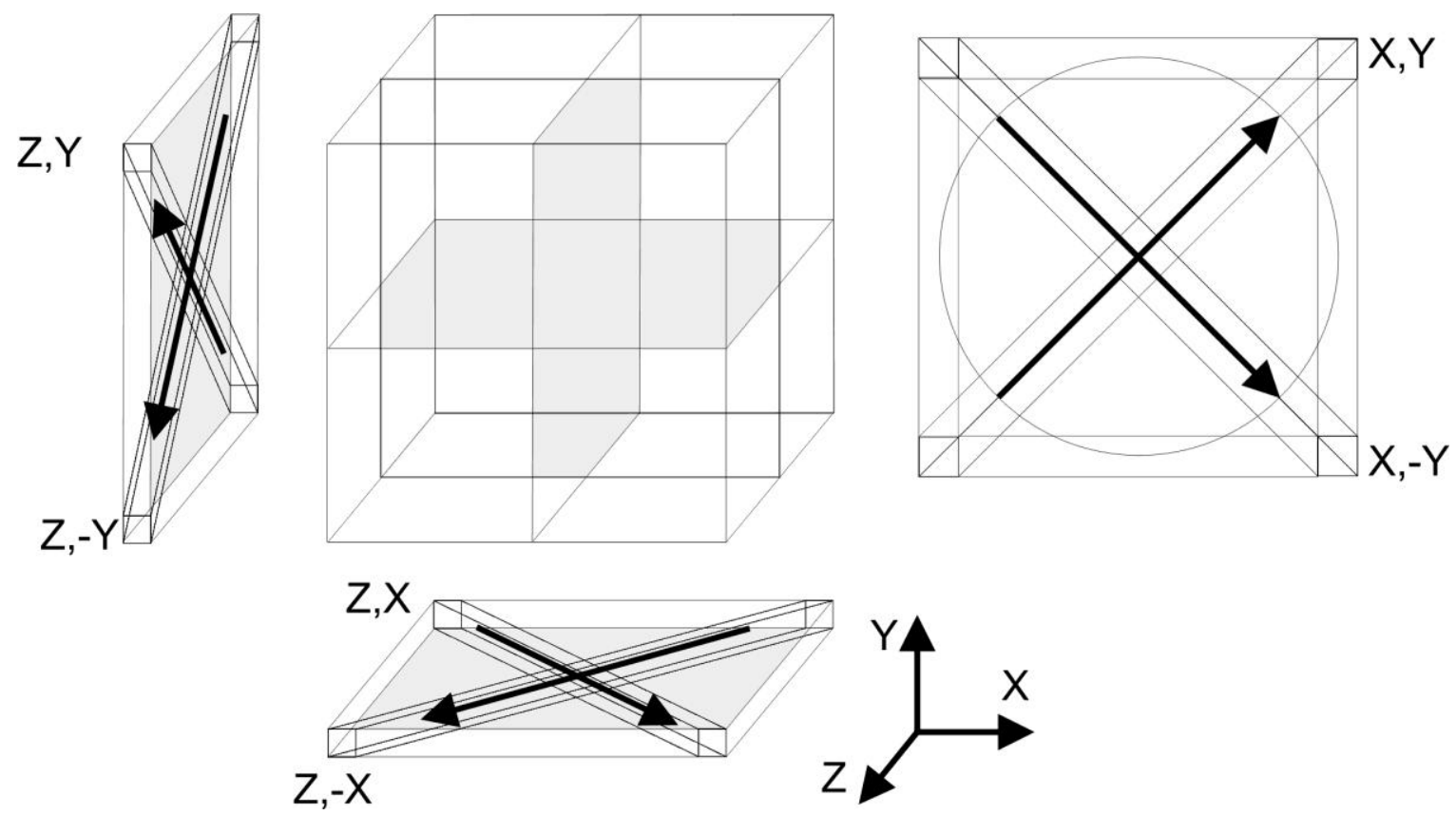

Figure 3 .1: Six linear field-map projections diagonally acquired along rectangular columns (sticks) defined by the intersection of the two orthogonal slices through the selected shim voxel. (Reproduced from ParaVision manual, Advanced Users Manual D-4: Fastmap) 


\subsubsection{Water Suppression}

In the brain tissues, the concentrations of brain metabolites are much lower than the concentration of water. Whereas the concentration of water protons is roughly $80 \mathrm{M}$, cerebral metabolite levels are on the order of $\mathrm{mM}$. Consequently, the localized ${ }^{1} \mathrm{H}$ MR spectrum is dominated by the signal from water. Hence, water suppression techniques are pivotal to observe the metabolite resonances in in vivo ${ }^{1} \mathrm{H}$ spectroscopy. Figure 3.2 shows a perfect example of a water-suppressed localized proton NMR spectrum of aqueous solution of GABA, using STEAM spectroscopy in comparison to that acquired without application of water suppression.
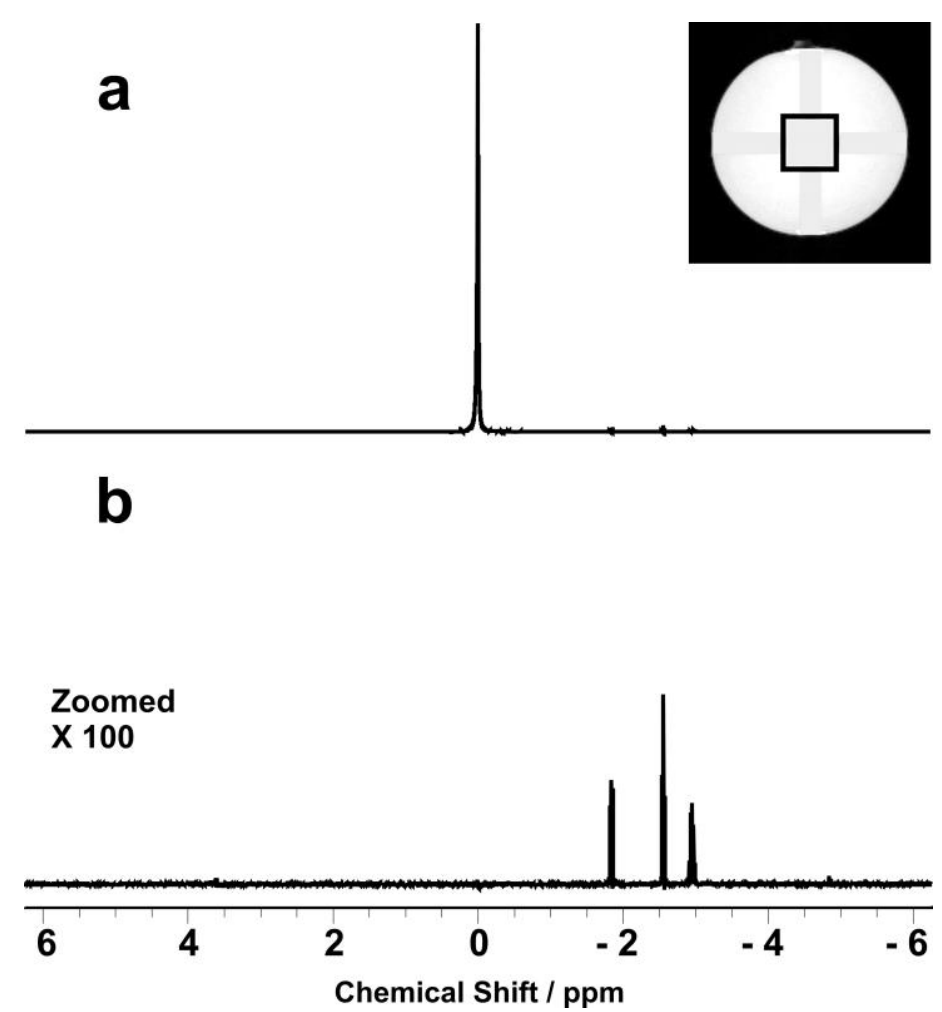

Figure 3.2: Localized proton NMR spectrum of an aqueous solution of $200 \mathrm{mM}$ GABA obtained without (a) and with (b) applying CHESS water suppression module. Axial RARE image (inset) illustrates the location of the VOI in the center of spherical phantom. The water signal intensity is reduced drastically. Accordingly, resolved proton resonances of GABA are clearly visible in the spectrum.

Incomplete water suppression will result in baseline distortion and, consequently, degrades the quantification certainty. A desirable water suppression 
method should reduce the water amplitude well below the metabolite amplitude, without overflowing the digitizer, for a limited dynamic range of analog-to-digital converters (ADCs) of the RF receiver channel. Moreover, this eliminates baseline distortions linked to residual water and prevents spurious signals such as sidebands aroused from system instabilities. However, a consistent amount of residual water can be readily exploited for phase and eddy current correction (Klose, 1990) or quantification of metabolite.

Several attempts have been made and many techniques have been suggested for more efficient suppression of water signal, mainly including relaxation based techniques, binomial based, frequency selective refocusing or excitation and frequency selective saturation.

WEFT (water eliminated Fourier transform) was initially developed on the basis of the difference in longitudinal $T_{1}$ Relaxation and employed in high resolution NMR (Patt and Sykes, 1972). This is similar to inversion recovery sequence, which consists of $180^{\circ}$ selective inversion pulse followed by a delay to main sequence. After $180^{\circ}$ pulse, a spoiler gradient is applied when water longitudinal magnetization recovered to its zero value, in order to dephase water signal prior to the localization pulse sequence. Therefore, metabolite magnetization is partially preserved during delay to excitation pulse. This is the reason why it is also considered to be an inversion nulling method. The result can be further enhanced in in vivo application for several $T_{1}$, using multiple inversion nulling with optimized delay (Berkelbach van der Sprenkel et al., 1992, Duijn et al., 1992). An alternate water suppression method for taking advantage of $T_{1}$ differences by using $90^{\circ}-\mathrm{t}-180^{\circ}-\mathrm{t}-90^{\circ}$ sequence was reported by Becker, in which $\mathrm{t}$ is considered as an inter-pulse delay (Becker et al., 1969, Shoup et al., 1972). Driven equilibrium Fourier transform (DEFT) has been introduced in the past as a method of signal enhancement for acquisitions with short repetition times in pulsed ${ }^{13} \mathrm{C}$ magnetic resonance spectroscopy. The appropriate selection of $\mathrm{t}$ brings about nulling of the longitudinal component of the water magnetization after inversion pulse whereas metabolite magnetization recovered before excitation pulse. However, in several respects, the use of $T_{1}$ based methods is disadvantageous for the suppression of water resonance in vivo. In particular, relatively minor differences of $T_{1}$ relaxation time between water and 
metabolite in the in vivo situation, lead to attenuation of metabolite as well, consequently resulting in unreliable metabolite quantification.

Additionally, beside the $\mathrm{T}_{1}$-selective method pointed out above, another category of water suppression techniques were established on binomial pulses, which originally have proven to be useful to achieve selective excitation for water suppression applications at high resolution NMR spectroscopy (Hore, 1983). Longitudinal magnetization of solvent is nullified in excitation profile of frequency-selective pulse trains, for example, DANTE RF pulses (delays alternating with nutation for tailored excitation) employs a series of rectangular (hard) pulses with short duration (Morris and Freeman, 1978). Albeit, it was recognized that periodical frequency response of these pulses may induce phase and amplitude modulations over the spectrum. Potential limitations can be stated for these approaches, namely, high power deposition due to using hard pulses but less than low power saturation of the water signal technique. Another weakness is the difficulties in utilizing in short echo time single shot localization sequences like STEAM or PRESS.

WATERGATE (water-suppression by gradient-tailored excitation) method is a way of achieving enhancement of the frequency profile generated by tailored selective excitation pulses, which has been presented by Piotto and Sklenar et al. (Piotto et al., 1992, Sklenar et al., 1993). In this approach, performance of binomial pulse in pulse train has been noticeably improved by numerical optimization of sub-pulses property such as inter-pulse intervals, pulse lengths and phases. However, Gradient field distortion, originating entirely from eddy currents, is considered as a potential problem for the methods employing pulse field gradients (Guéron and Plateau, 2007).

Along with all of the aforementioned methods, presaturation of the water signal in in vivo MRS can be accomplished by chemical shift selective (CHESS) (Haase et al., 1985) saturation, which is one of the most robust and popular approaches for water suppression. Figure 3.3 shows a schematic diagram of CHESS water suppression, which was employed in this thesis. Three successive frequency-selective excitation rf pulse and associated spoiler gradients are instantly applied, prior to localization pulse sequence. Various versions of CHESS have been established to result in further improvement in suppression performance. By employing more than one CHESS cycle (Frahm et al., 1990) 
and optimization of corresponding Gaussian pulses flip angle (Webb et al., 1994, Ernst and Hennig, 1995), suppression factor of more $>1000$ can be typically achieved over a range of $\mathrm{T}_{1}$ relaxation times.
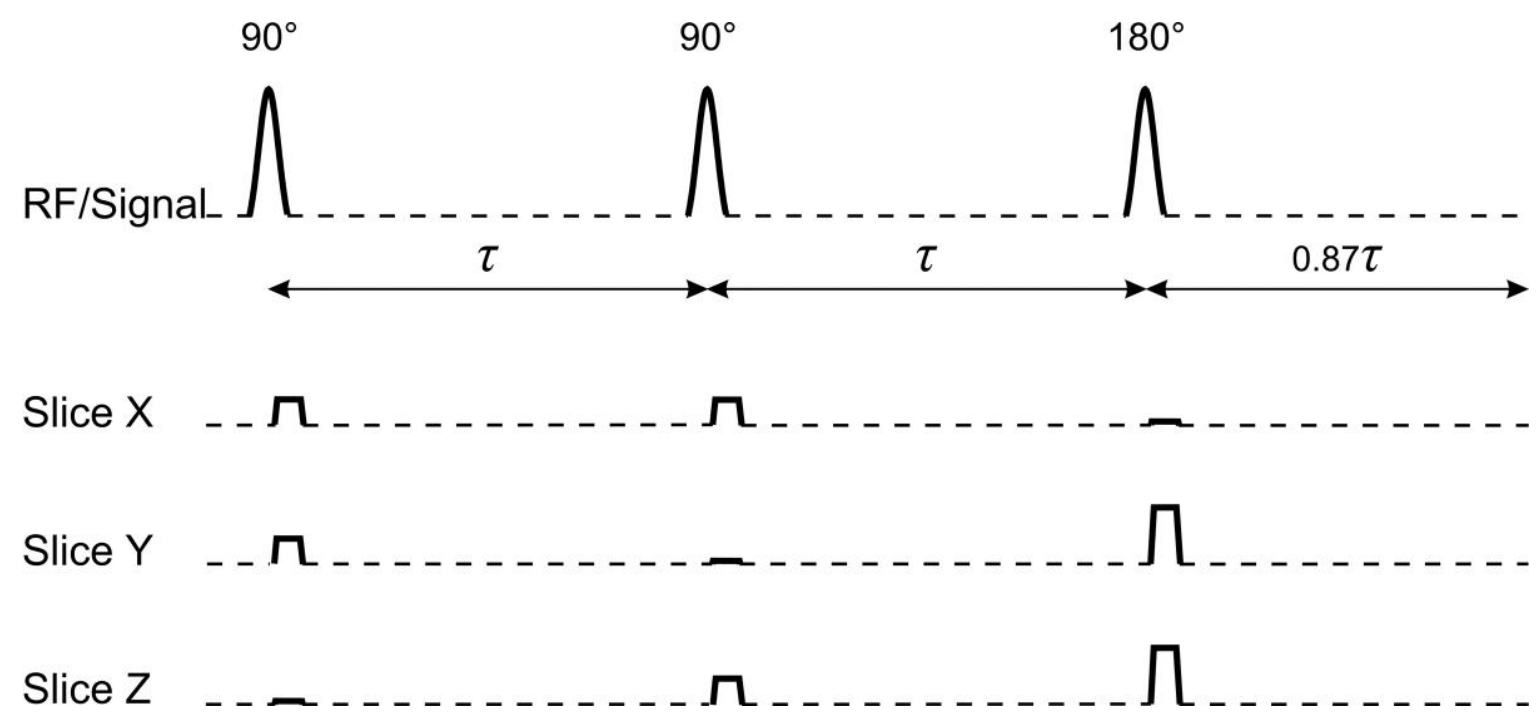

Figure 3.3: Schematic illustration of the chemical-shift-selective (CHESS) water suppression sequence used for localized proton spectroscopy of mouse brain at high magnetic field (9.4 T). Sequence comprised of three successive Gaussian pulses of duration $7.83 \mathrm{~ms}$, giving a saturation bandwidth of $350 \mathrm{~Hz}$ together with associated spoiler gradients. The interleaved outer volume suppression blocks are not shown, but are applied after each CHESS element. $\tau$ is the inter-pulse delay.

DRYSTEAM (drastic reduction of water signals in spectroscopy with the stimulated echo acquisition mode) additionally utilizes CHESS pulses during mixing time (TM) of STEAM sequence when the magnetization vector is longitudinal (Moonen and Vanzijl, 1990). However, this approach was not pursued in the present work, since mixing time was kept short to avoid signal attenuation caused by subject motion or as a result of diffusion in the period between the two $B_{0}$ magnetic field gradients in TE (Frahm et al., 1990).

Likewise, to reduce the impact of longitudinal relaxation decay during $T M$, which might introduce complications on spectral quantification, other variants of CHESS method have also been proposed by applying more than three CHESS cycles. In a variant known as WET (water suppression enhanced through $\mathrm{T}_{1}$ effects), Ogg et al. attained $\mathrm{T}_{1^{-}}$ and $B_{1}$-insensitive suppression by utilizing four Gaussian pulse with numerically 
optimized flip angle (Ogg et al., 1994). Another suppression method to further circumvent the disadvantages of sensitivity to $B_{1}$ and $T_{1}$ was proposed (Tkáć et al., 1999). VAPOR (variable pulse power and optimized relaxation) applies seven asymmetrical and numerically optimized RF pulses. It is important to bear in mind that the increased number of CHESS elements may increase the risk of occurrence of unwanted echoes, due to using a number of RF and gradient pulses. Moreover, magnetization exchange with water protons, as a result of long water suppression scheme, can attenuate methyl signal of creatine. Therefore, the suppression scheme in the current work only employed three CHESS RF pulses, based primarily on the properties of Ernst et al (Ernst and Hennig, 1995).

One of the primary goals of this study was to find out the proper water suppression parameters (e.g., RF shape, band width, inter-pulse delay, and spoiler gradient strength) at $9.4 \mathrm{~T}$ in such a way that any important information on metabolite resonances in the typical spectral range (up to 4.2 PPM) will not be lost. To achieve this goal, the influence of each parameter on the applied water suppression performance was systematically investigated for optimization. The effectiveness of suppression was verified in both phantom and for mouse brain in vivo.

\subsubsection{Outer Volume Suppression (OVS)}

In short, echo time localized spectroscopy, particularly in cortical regions, intense lipid signal predominantly stemming from skull with short spin-spin relaxation time, can obscure metabolites resonances such as lactate and alanine, owing to their spectral overlap. On the other hand, concerning the localization performance quality of sequence, one should keep in mind that employing a larger crusher gradient may introduce eddy current artifact to spectrum, while insufficient spoiling may raise the chance for occurrence of unwanted coherences from outside of volume of interest, like those arising from regions with inferior field homogeneity (i.e. mouth or sinuses). In this work, the effects of different strategies for mitigating possible signal contamination, originating from outside of the selected volume, were investigated. 


\subsubsection{The relative detectability of strongly coupled} metabolite resonances in proton MR spectra at low- and high-field strength

Most major cerebral metabolites are detectable in short-echo time proton MRS at both low (e.g., 2.35-4.7 T) and high field strengths ( $\geq 7.0 \mathrm{~T}$ ). High fields certainly offer advantages in terms of SNR and, therefore, allow for the use of reasonably small volumes-of-interest (VOI). In addition, the precision of metabolite quantification and detectability of weakly represented metabolites has been reported to increase substantially at 7T, relative to 4T (Tkáć et al., 2009). On the other hand, a potential merging of multiplet signal pattern may offer a better relative detectability of its respective resonance at low magnetic fields. The purpose of this work was to evaluate the relative detectability of strongly coupled metabolites at $2.35 \mathrm{~T}$ and $9.4 \mathrm{~T}$, by comparing their respective peak intensities to those of uncoupled singlet resonances. ${ }^{1}$ The results further clarify the relative merits of field strengths, as discussed in previous publications (Michaelis et al., 1991, Michaelis and Frahm, 2005, Tkáć and Gruetter, 2005)

${ }^{1}$ A part of this work was accepted for an e-Poster presentation at the 25th Annual Meeting of the European Society of Magnetic Resonance in Medicine and Biology, Valencia, October 2008 and also was appeared in a review article by Michaelis et al. MICHAELIS, T., BORETIUS, S. \& FRAHM, J. (2009) Localized proton MRS of animal brain in vivo: Models of human disorders. Progress in Nuclear Magnetic Resonance Spectroscopy, In Press, Corrected Proof. 


\subsection{Methods}

\subsubsection{FASTMAP}

In the present study, to achieve high spectral resolution, nonlinear local field inhomogeneities were automatically corrected by adjustment of second-order shim coils.

Optimisation of static magnetic field homogeneity (i.e. shimming) was accomplished by using FASTMAP. Field homogeneity further improved prior spectroscopic measurements by using optimised values for FASTMAP sequence parameters. For a $3 \mathrm{~mm}$ cubic voxel of interest, stick size of $1.25 \mathrm{~mm}$, repetition time of $1000 \mathrm{~ms}$, spectral bandwidth of $5000 \mathrm{~Hz}$, and acquisition averages of 2 were set, such that the whole adjustment of all linear and quadratic shim coils was accomplished within $2 \min$.

\subsubsection{Water Suppression}

As discussed earlier in chapter 3.1.2., the current version of water suppression consists of three successive CHESS pulses (90 90 180), each of which is followed by associated spoiler gradients, as has been proposed earlier by (Frahm et al., 1990, Moonen and Vanzijl, 1990, Ernst and Hennig, 1995), preceding the STEAM localization sequence.

As stated earlier, the RF amplitude of the CHESS-type water suppression pulses and, therefore, the overall water suppression performance could be affected by the RF homogeneity profile of the transmitter coil $\left(B_{1}\right)$, as well as the magnetic field homogeneity $\left(B_{0}\right)$ within the voxel. Susceptibility to $B_{1}$ inhomogeneities was considerably alleviated in this work by using a birdcage volume resonator, which allows the generation of relatively uniform RF fields together with the advantage of providing whole-head coverage, although it still depends on exact calibration of the $90^{\circ}$ pulse power. The influence of $B_{0}$ inhomogeneities was reduced by automated, localized 
shimming of all first- and second-order terms using FASTMAP (fast automatic shimming technique by mapping along projections) (Gruetter, 1993, Gruetter and Tkáć, 2000) for each volume of interest (VOI). In all measurements, efficiency of CHESS pulses was optimized with respect to flip angle of the RF pulses after localized shimming, by minimizing the signal intensity of residual water in the volume of interest (VOI), just prior to final acquisition.

The efficiency of the current water suppression method was carefully optimized on a phantom containing aqueous model solutions of cerebral metabolites, with regard to our experimental setup. In addition, performance was monitored and assessed in vivo, with respect to CHESS sequence parameters, in order to guarantee sufficient suppression over different regions of mouse brain.

As mentioned earlier, increasing the gradient amplitude would pose a potential risk of introducing artifact to the spectrum, due to eddy current effects, without improving the suppression performance. Thus, prior to the optimization of the band width and the inter-pulse delay, the influence of the amplitude of the spoiler gradient in the CHESS sequence on water suppression was investigated in a pilot study. The strength of the applied gradients was incremented from $80 \mathrm{mTm}^{-1}$ to 160,240 and $320 \mathrm{mTm}^{-1}$, whereas the duration, orientation and polarity remained constant. In vivo magnetic resonance spectra obtained from mouse brain were evaluated by considering the residual water signal height, as well as the quality of spectra. It turned out that the linewidth, baseline and phase remain unaffected by increasing the gradient spoiling powers. No significant change was observed for residual water resonance. Based on these results, minimum spoiling capacity was chosen because increasing the gradient amplitude may cause artifacts due to eddy current effects. Nevertheless, it ensured a sufficient dispersion of transverse water coherences, which guarantees robust water suppression as well as consistent spectral quality. 


\section{Bandwidth}

A series of localized water suppressed proton spectrum with varied bandwidth of Gaussian RF pulses were obtained from the centre of spherical phantom of a $10 \mathrm{~mm}$, containing a mixture of $\mathrm{Lac}$ and $\mathrm{Cr}(1: 2)$. All the other experimental parameters were kept unchanged during measurement. The spectra scaled individually to methyl signal of creatine, which was considered unaffected from suppression. Methine quartet of lactate, as a resonance of interest, were evaluated to determine the effective water suppression bandwidth.

Influence of this parameter on water suppression performance and critical resonances in the vicinity of the water signal were also evaluated in vivo. $8 \mathrm{ml}$ VOI was selected in midbrain and all spectra were measured using identical acquisition parameters as those used for the phantom experiment, except that repetition time was set to $6 \mathrm{sec}$ (see Chapter 2.3 for more details). The measurement repeated several times with continuous change of a selected parameter, e.g. RF bandwidth in CHESS water suppression sequence. Absorption (real) spectra were manually phased and reported directly, without baseline correction or resolution enhancement.

\section{Inter-pulse delay}

After finding the optimal bandwidth for CHESS RF pulses, the influence of different intervals between the RF pulses in the current water suppression scheme was assessed. This was accomplished by varying the length of the corresponding inter-pulse delay between CHESS RF pulses in a series of experiments, without altering measurement parameters. Quality of water suppression was verified in a phantom, as mentioned previously, by comparing the signal intensity of the residual water obtained at different inter-pulse delays. Fully relaxed spectra ( $T R=10000 \mathrm{~ms}$ ) were acquired from a $125 \mu \mathrm{L}\left(5 \times 5 \times 5 \mathrm{~mm}^{3}\right)$ volume of interest (VOI). Interval values were varied from 25 to 225 with increments of 25 ms (part of data not shown for simplicity), whereas water suppression bandwidth was kept constant at $350 \mathrm{~Hz}$ (TE $=10 \mathrm{~ms}, 8$ scans). Spectra were individually scaled to the intensity of the methyl signal of creatine as a reference. 
This procedure was repeated to verify suppression performance in a in vivo condition by analysis of a series of spectrum, measured by using the same parameter for CHESS sequence.

Settings of the delays were verified by the analysis of a mouse brain spectrum. In vivo ${ }^{1} \mathrm{H}$ NMR spectra of the healthy mouse brain were measured using STEAM with TE = $10 \mathrm{~ms}$. A VOI size of $2.7 \times 2.3 \times 2.5 \mathrm{~mm}$ localized in the central thalamus. In the final version of water suppression, utilizing Gaussian pulses of duration $7.83 \mathrm{~ms}$, with bandwidths of $350 \mathrm{~Hz}$ and interleaved outer volume suppression module enabled a minimum inter-pulse delay of 50 ms. Overall duration of the CHESS module was $147 \mathrm{~ms}$. The inter-pulse delay was incremented as indicated in the Figure 3.9, while keeping the suppression bandwidth constant $(350 \mathrm{~Hz})$.

For each measurement, Flip angle adjustment was performed to balance the effect of $T_{1}$ relaxation (Moonen and Vanzijl, 1990). Choosing the shortest feasible interpulse delay enhanced $T_{1}$ insensitivity, in addition to shortening the entire water suppression module.

The current water suppression scheme $\{\tau, \tau, 0.87 \tau ; \theta, \theta, 2 \theta\}$, suggested by Ernest et al (Ernst and Hennig, 1995), was compared to that of equidistant timing type $\{\tau, \tau, \tau ; \theta$, $\theta, 2 \theta\}$. Therefore, only the third inter-pulse delay (the interval between the third RF pulse and the start of the localization sequence) differed in two sequences. The values of 75,100 , and 125 ms were chosen for $\tau$ in CHESS sequence, while the bandwidth of the RF pulses were kept constant. Then the spectra were compared to those obtained with $0.87 \tau$ for the third inter-pulse delay. Spectra were acquired with $15 \mathrm{ml}$ VOI $(2.5 \times 2.0 \times$ $3.0 \mathrm{~mm}^{3}$ ) position in the mid brain, within measuring times of $4.3 \mathrm{~min}$ each (TR 8,000 ms, 32 scans). Spectra were processed in magnitude mode and analytically compared on the basis of the ratio of intensities of residual water peak, over that of methyl resonance of creatine, as reference. 


\subsubsection{Outer Volume Suppression (OVS)}

Problems associated with the suppression of lipid signals from subcutaneous fatty tissues have been tackled by various strategies in this work:

By means of oblique volume of interest, the excitation volume was positioned confined to brain regions - especially in the cortical area - in order to exclude subcutaneous fat. Localization performance was improved, using adequate amounts of crusher gradients to dephase unwanted signal contamination from outside the VOI, in conjunction with employing broad band selective RF pulses to alleviate the volume misregistration problem, additionally considering a proper applied excitation order of coronal-axial- sagittal in pulse sequence and thirdly, placing spatial saturation bands, precisely surrounding the volume to suppress any confounding signal (Connelly et al., 1988, Duyn et al., 1993, Posse et al., 1993, Shungu and Glickson, 1993).

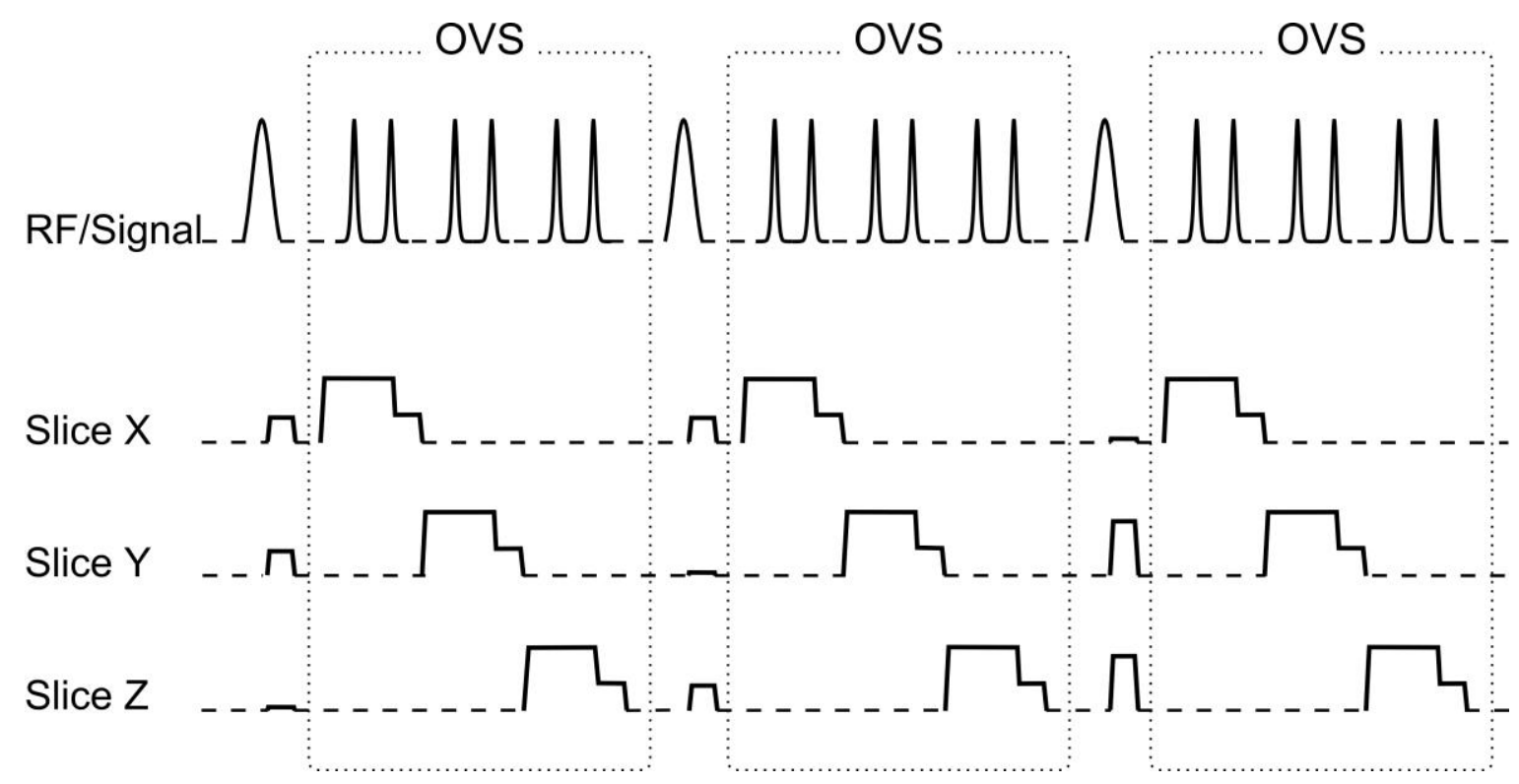

Figure 3.4: Schematic illustration of the employed three outer volume suppression (OVS) blocks (marked by the dashed line) used to reduce contamination arising from outside of volume of interest. OVS block applied after each CHESS element and comprised of six 1.0 msec hyperbolic secant RF pulses. Each pair of pulses selected the sides of the voxel in different directions. 
On the latter approach, excitation of magnetization from unwanted regions of the brain was followed by rapid dephasing, pursuant to applied crusher gradients. In this work, CHESS water suppression pulses were interleaved with three blocks of outer volume suppression, as illustrated in Figure 3.4. Hence, the improvement in localization performance was particularly obtained in the regions closest to the skull The OVS comprised of $37 \mathrm{msec}$ blocks with six $1.0 \mathrm{msec}$ full-passage hyperbolic secant band-selective pulses, with $90^{\circ}$ nominal flip angle and a bandwidth of $20 \mathrm{kHz}$ in each, followed by a crusher of $10 \mathrm{msec}$ of amplitude $40 \mathrm{mTm}^{-1}$. The OVS module extended over $3 \mathrm{~mm}$ around the VOI, with a 0-mm gap to the voxel. The first two pulses selected the sides of the voxel in the right-left direction, and the second and third pairs selected the anterior-posterior and head-feet directions, respectively. 


\subsubsection{The relative detectability of strongly coupled} metabolite resonances in proton MR spectra at low- and high-field strength

Fully relaxed, localized proton MRS (STEAM) was performed at 2.35 T and 9.4 T. Solutions of metabolite mixtures of Ins: $\mathrm{Cr}(50: 50 \mathrm{mM}), \mathrm{Glc}: \mathrm{Cr}(50: 50 \mathrm{mM})$ and NAA:Glu:GIn (50:50:25 mM) were investigated in vitro. Line broadened in vitro MR spectra ( $6 \mathrm{~Hz}$ and $12 \mathrm{~Hz}$ for $2.35 \mathrm{~T}$ and $9.4 \mathrm{~T}$, respectively) were compared to MR spectra of NMRI mouse brain in vivo. Line-broadened versions of spectra were produced by multiplying exponential decay function ( $12 \mathrm{~Hz}$ and $6 \mathrm{~Hz}$ for $9.4 \mathrm{~T}$ and $2.35 \mathrm{~T}$, respectively) to render them representative of limited spectral resolution in vivo (which is reflected in e.g., broaden line-width) at each field strength, with a modification to the method described previously (Michaelis et al., 1991).

2.35 T: MRBR 4.7/400 mm magnet (Magnex Scientific, Abingdon, England), AVANCE II (Bruker BioSpin, Ettlingen, Germany). In vitro MRS was performed with a 10 $\mathrm{cm}$ Helmholtz transmit/receive coil (TR/TE/TM = 10000/10/10 ms, $10 \times 10 \times 10 \mathrm{~mm}^{3}, 64$ accumulations). In vivo MRS was performed with a $10 \mathrm{~cm}$ Helmholtz coil for RF excitation in conjunction with a $16 \mathrm{~mm}$ surface coil for signal reception (TR/TE/TM =6000/20/10 $\mathrm{ms}, 4.0 \times 3.0 \times 4.0 \mathrm{~mm}^{3}$ ) in a central position of the forebrain (512 accumulations).

9.4 T: 94/30 USR BioSpec, AVANCE II (Bruker BioSpin, Ettlingen, Germany). In vitro MRS was performed with a $72 \mathrm{~mm}$ quadrature birdcage transmit/receive coil (TR/TE/TM = 15000/10/10 ms, $8 \times 8 \times 8 \mathrm{~mm}^{3}, 16$ accumulations). In vivo MRS was performed with a $72 \mathrm{~mm}$ quadrature birdcage coil for signal excitation and a quadrature mouse brain surface coil for signal reception (TR/TE/TM $=6000 / 10 / 10 \mathrm{~ms}$ ) in a central position of the forebrain $\left(4.0 \times 3.0 \times 4.0 \mathrm{~mm}^{3}, 32\right.$ accumulations $)$ and in the hippocampus ( $2.0 \times 1.2 \times 2.2 \mathrm{~mm}^{3}, 128$ accumulations $)$. 


\subsection{Results}

\subsubsection{FASTMAP}

Figure 3.10 elucidates the putative role of localized second-order shimming to provide superior spectral resolution and sensitivity, compared to the one where only global shimming were applied, to refine the static field. In addition, uncompensated inhomogeneity of the $B_{0}$ magnetic field results in imperfect water suppression. This is largely due to the frequency-selective mechanism of employed CHESS pulses. Efficient shim system (shim coils and shim drivers) was capable of producing maximum shim strengths of $X=8741 \mathrm{~Hz} / \mathrm{cm}, Y=8715 \mathrm{~Hz} / \mathrm{cm}$, and $Z=8651 \mathrm{~Hz} / \mathrm{cm}$ for the linear shim terms and X2_Y2 $=698 \mathrm{~Hz} / \mathrm{cm}^{2}, Z X=3715 \mathrm{~Hz} / \mathrm{cm}^{2}, Z 2=1243 \mathrm{~Hz} / \mathrm{cm}^{2}, \mathrm{ZY}=2466 \mathrm{~Hz} / \mathrm{cm}^{2}$, $X Y=557 \mathrm{~Hz} / \mathrm{cm}^{2}$ for the second-order ones. Optimal application of this shim system resulted in the linewidths (FWHM) of 11-14 Hz for the unsuppressed water signals from most of the brain regions of mice in vivo. For most metabolites in aqueous solutions (27 $\mathrm{ml})$, reproducible water linewidths of $1.5-2.0 \mathrm{~Hz}$ were achieved. 


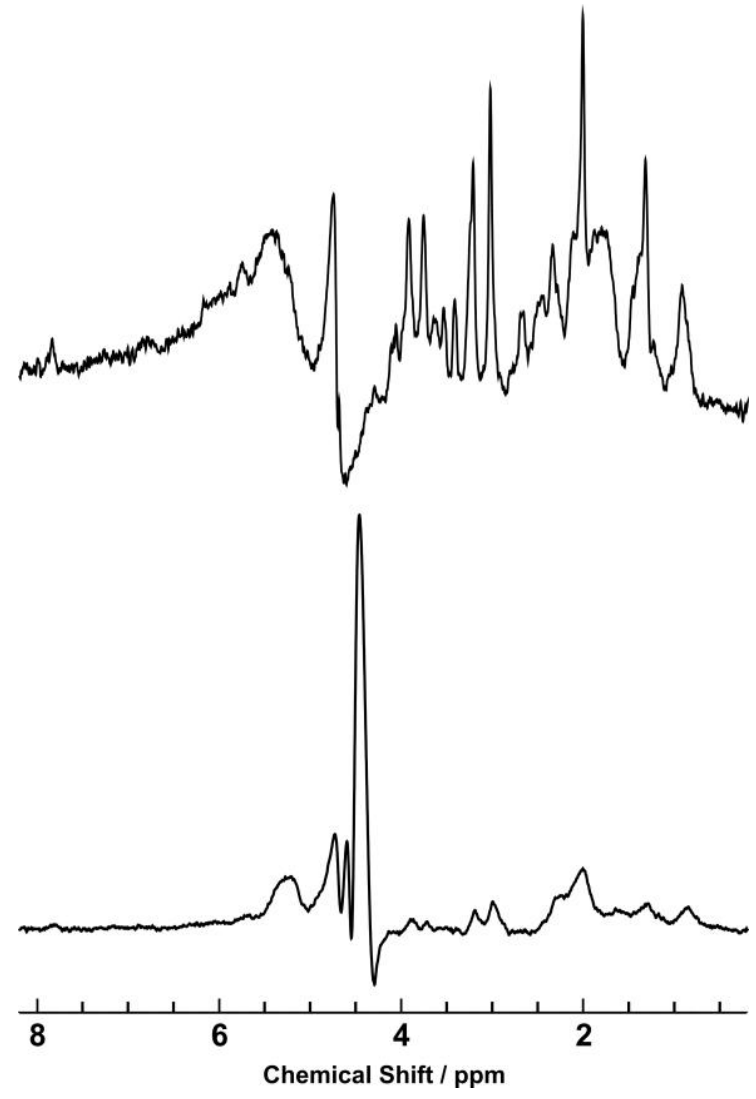

Figure 3.5: The effect of high-order shimming on spectral resolution and sensitivity of spectrum, obtained using localized proton MRS (STEAM, TR/TE/TM=6000/10/10 ms, $15.5 \mu \mathrm{l}$ VOI, $2 \times 128$ accumulations) from the thalamus of a mouse in vivo at 9.4 T. The suppression of the water signal after second-order shimming using FASTMAP (top) is clearly superior to that without it (bottom, only with global shimming). Each spectrum is individually scaled to the largest peak for comparison.

\subsubsection{Water Suppression}

\section{Bandwidth}

Figure 3.6 depicts proton spectra of a mixture of lactate (Lac) and creatine ( $\mathrm{Cr}$ ), acquired with different bandwidths of Gaussian-shaped CHESS pulses. In order to eliminate the problem arising from different phasing, the spectra were presented in magnitude mode and shifted along the chemical shift axis to a different degree for a better visualization of suppression effect. Residual water signal served as chemical shift reference. Comparison of the quartet signal of Lac shows a decrease in amplitude when the suppression band width of 


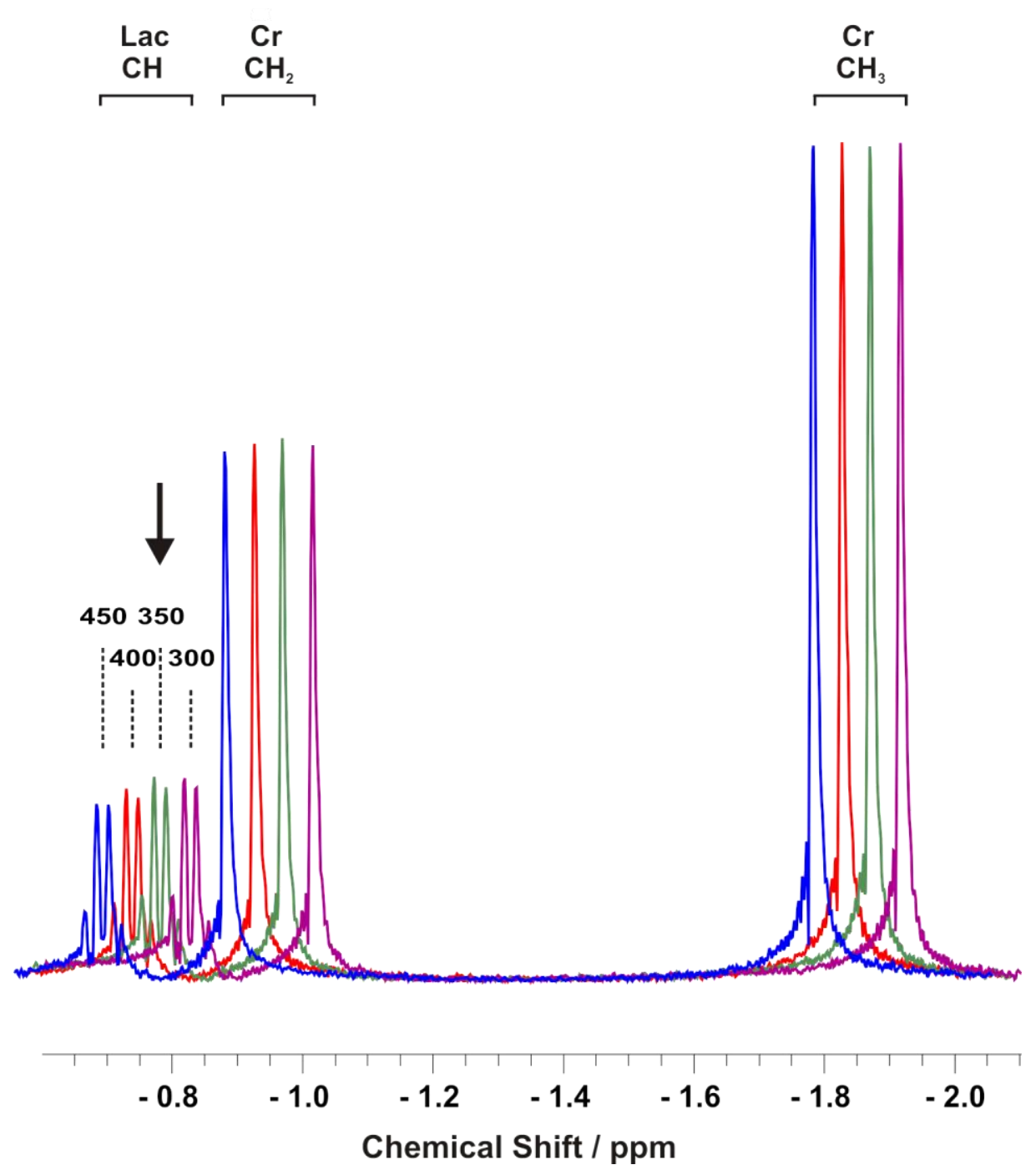

Figure 3.6: Proton NMR spectra of a 1:1 mixture of lactate (Lac) and creatine $(\mathrm{Cr})$ at different water suppression bandwidths ranging from 300 to $450 \mathrm{~Hz}$. $\left(9.4 \mathrm{~T}\right.$, VOI $5 \times 5 \times 5 \mathrm{~mm}^{3}, \mathrm{TR} / \mathrm{TE} / \mathrm{TM}=10,000 / 10 / 10$ ms, 32 transients) displayed in magnitude mode. For a better visualization of the results, the spectra acquired with 300,400 , and $450 \mathrm{~Hz}$ bandwidth were shifted along the chemical shift axis by $-15,+15$, and $+30 \mathrm{~Hz}$, respectively. Chemical shifts are given in parts per million (ppm) and referenced to residual water signal. While the heights of the quartet signal of Lac at $350 \mathrm{~Hz}$ (arrow) remain unchanged compared to those at $300 \mathrm{~Hz}$, a decline of Lac can be seen in spectrum obtained with water suppression band width more than $350 \mathrm{~Hz}$. 
greater than $350 \mathrm{~Hz}$ is applied. The chemical shift difference between the water resonance and methine quartet of lactate in in vitro is equivalent to that of water and methylene singlet resonance of creatine in in vivo condition. Therefore, as it can be concluded from Figure 3.7, $\mathrm{CH}_{2}$ resonance of creatine remained intact from attenuation, by choosing $350 \mathrm{~Hz}$ bandwidth for CHESS pulses, while water signal was eliminated efficiently.
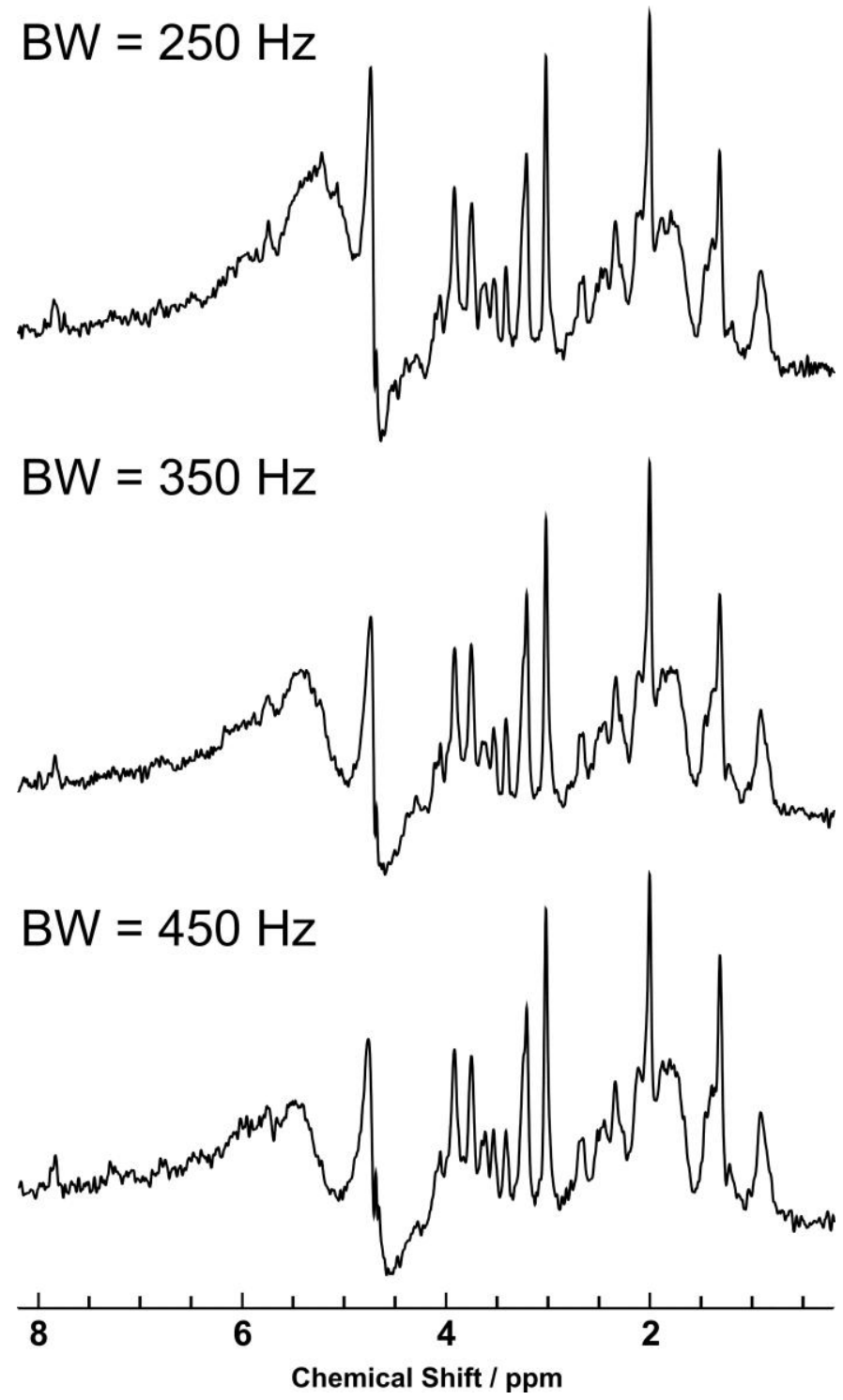

Figure 3.7: In vivo water-suppressed ${ }^{1} \mathrm{H}$ MR spectra of the mouse brain measured using STEAM with different bandwidth of CHESS pulses to demonstrate effect of this parameter on overall water suppression. Other parameters: $9.4 \mathrm{~T}$, VOI $40 \times 25 \times 30 \mathrm{~mm}^{3}, \mathrm{TR}=6000 \mathrm{~ms}, 256$ accumulation. The full chemical shift range of the spectra is shown to demonstrate the accessible water suppression under the experimental conditions used. 


\section{Inter-pulse delay}

The series of water-suppressed proton spectra in Figure 3.8 demonstrates the inter-pulse delay dependence of CHESS water suppression sequence. Despite the marked changes that occurred in residual water in shorter inter-pulse delay, no changes in the peak height of Lac and $\mathrm{Cr}$ resonances were observed.

\section{$50 \mathrm{~ms}$}

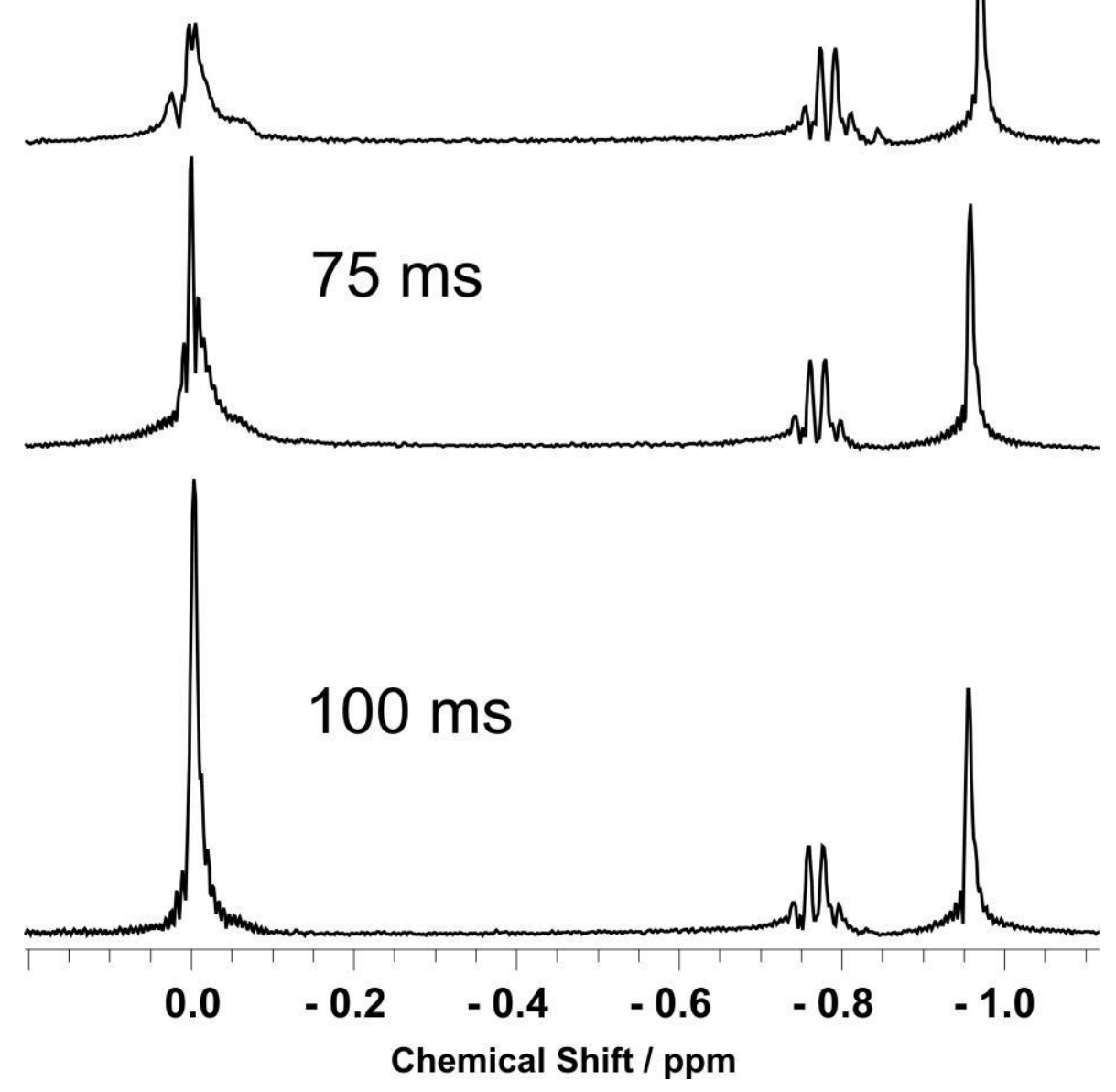

Figure 3.8: Proton NMR spectra of a 1:1 mixture of lactate (Lac) and creatine $(\mathrm{Cr})$ at different inter-pulse delay of CHESS water suppression sequence. (9.4 T, VOI $5 \times 5 \times 5 \mathrm{~mm}^{3}, \mathrm{TE}=10 \mathrm{~ms}, \mathrm{TR}=10,000 \mathrm{~ms}, 8 \mathrm{scans}$ ). interval value was varied from 25 to 225 with increment of 25 ms (part of data not shown for simplicity) whereas water suppression bandwidth was kept constant at $350 \mathrm{~Hz}$. Magnitude spectra presented to obviate any phase error from the signal and scaled to the creatine $(\mathrm{Cr})$ intensity for comparison. Chemical shifts are given in parts per million ( $\mathrm{ppm})$ and referenced to residual water signal. Improved water suppression was observed in spectrum obtained with the shortest possible inter-pulse delay of CHESS pulses. 
As can be seen from the residual water signals in Figure 3.9, the same holds for in vivo condition, but is not quite as salient as observed in the phantom study. The minimum possible inter-pulse delay of $50 \mathrm{~ms}$, considering the time required for the interleaved outer volume suppression (OVS) part of the sequence, gave rise to sufficient suppression of water resonance.

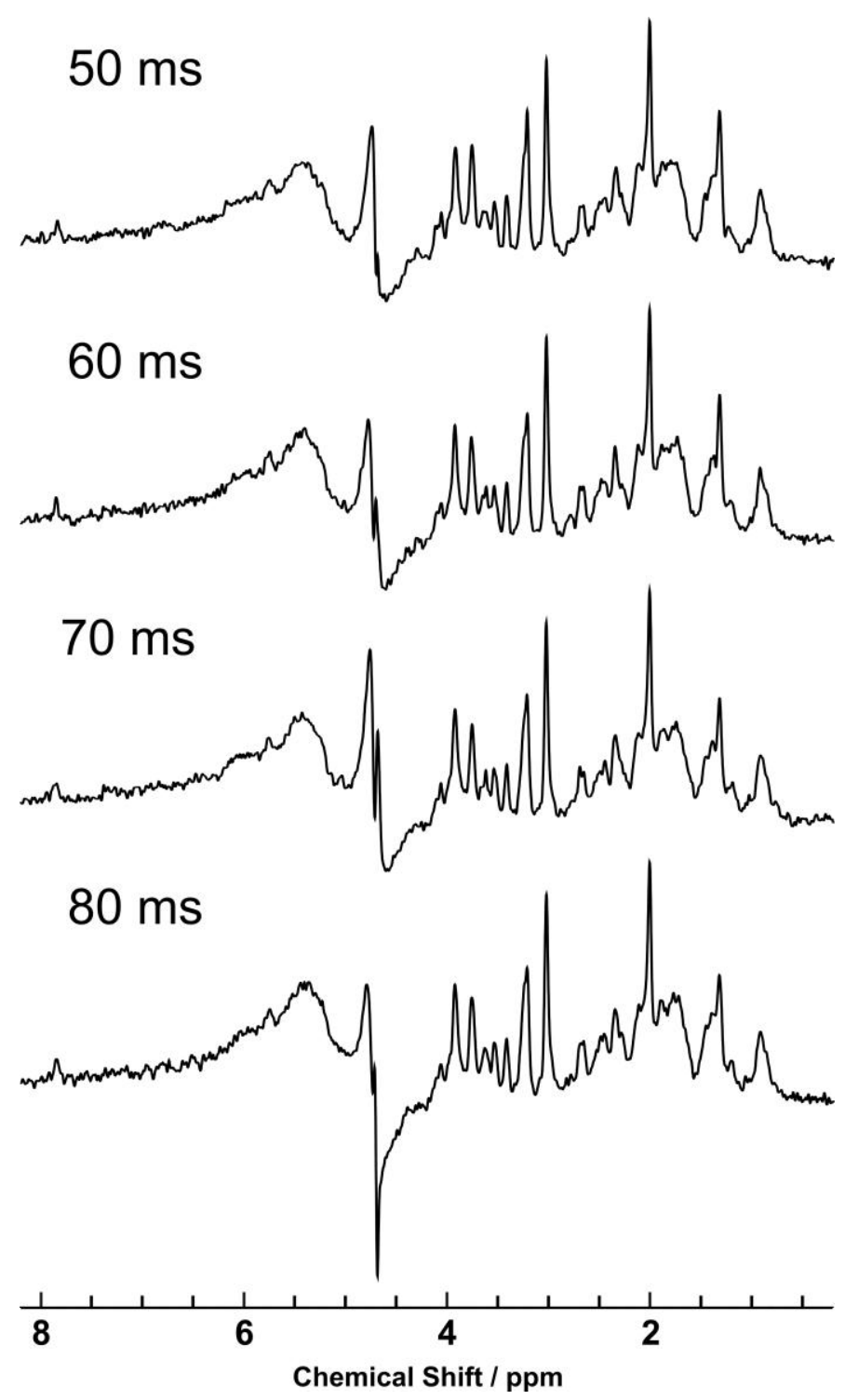

Figure 3.9: In vivo water-suppressed ${ }^{1} \mathrm{H}$ MR spectra of the mouse brain measured using STEAM with different inter-pulse delay of CHESS water suppression sequence to demonstrate effect of this parameter on overall water suppression. Other parameters: $9.4 \mathrm{~T}$, VOI $2.7 \times 2.3 \times 2.5 \mathrm{~mm}^{3}, \mathrm{TR}=6000 \mathrm{~ms}, 256$ accumulation. The full chemical shift range of the spectra is shown to demonstrate the accessible water suppression under the experimental conditions used. 
It is of note that no significant difference in suppression performance was observed between the equidistant timing scheme and the fined tuned one $\{\tau, \tau, 0.87 \tau\}$ at all selected inter-pulse delay $(\tau)$.

\subsubsection{Outer Volume Suppression (OVS)}

In close analogy to the CHESS, by repeating the OVS module three times, the sensitivity to $B_{1}$ field inhomogeneity was reduced and therefore, guarantees a successful elimination of spurious signal from outside the desired region of interest, predominantly from extracranial lipids (see Fig. 3.10).
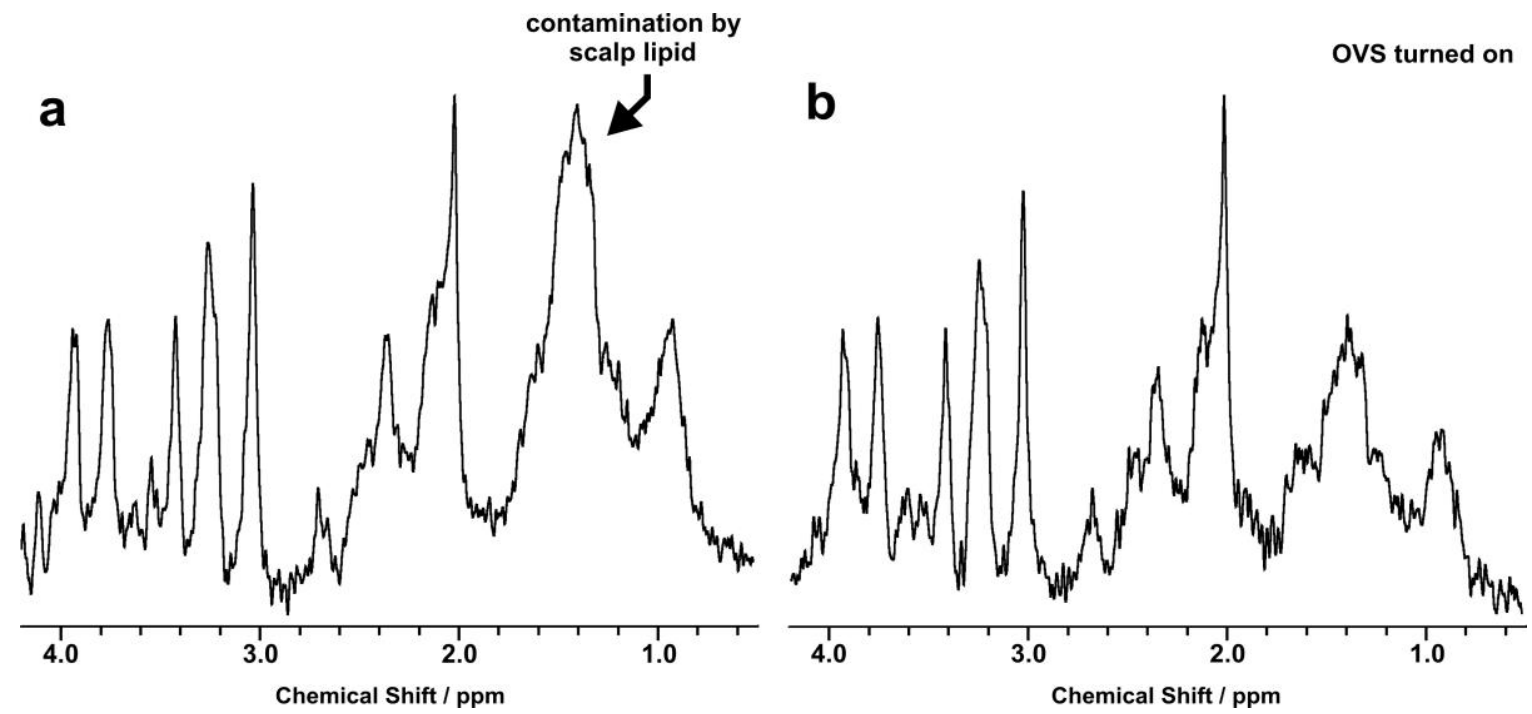

Figure 3.10: Effect of application of outer volume suppression (OVS) on localization performance and spectral quality. Localized proton MRS $\left(9.4 \mathrm{~T}\right.$, STEAM, TR/TE $=6000 / 10 \mathrm{~ms}, 3.0 \times 1.2 \times 3.0 \mathrm{~mm}^{3}, 64$ accumulations) from cortical region of healthy mouse brain (a) in comparison to that obtained with ovs (b). 


\subsubsection{The relative detectability of strongly coupled} metabolite resonances in proton MR spectra at low-and high-field strength

\section{Phantom Experiments}

Inositol

Proton MR spectra of a mixture of Ins and $\mathrm{Cr}$ obtained at both field strengths, with and without line broadening, are depicted in Figure 3.11. A doublet-of-doublet centered at $3.52 \mathrm{ppm}$ and a triplet at $3.61 \mathrm{ppm}$ are the two prominent multiplets of Ins at 9.4 $\mathrm{T}$ and each of them correspond to two protons. This complex multiplet resonance, merges into an apparent singlet resonance at $2.35 \mathrm{~T}$. As a consequence, the peak intensity ratio of Ins to $\mathrm{Cr}$ at $2.35 \mathrm{~T}$ is decreased by about $50 \%$ at $9.4 \mathrm{~T}$, after line broadening to in vivo conditions.

In addition, the intensity of the triplet at $3.27 \mathrm{ppm}$, originating from the ${ }^{5} \mathrm{CH}$ proton, reduced significantly at $2.35 \mathrm{~T}$. This triplet is typically obscured by the prominent singlet of the trimethylamine group of choline-containing compounds (Cho) at $3.21 \mathrm{ppm}$ and the triplet of taurine (Tau) centered at $3.25 \mathrm{ppm}$. The ${ }^{2} \mathrm{CH}$ triplet at $4.05 \mathrm{ppm}$ remains at 2.35 $\mathrm{T}$, however, it overlaps with the methine quartet of lactate and could be influenced by inadequate selection of water suppression bandwidth (Cerdán et al., 1985). 


\section{Cr:Ins}
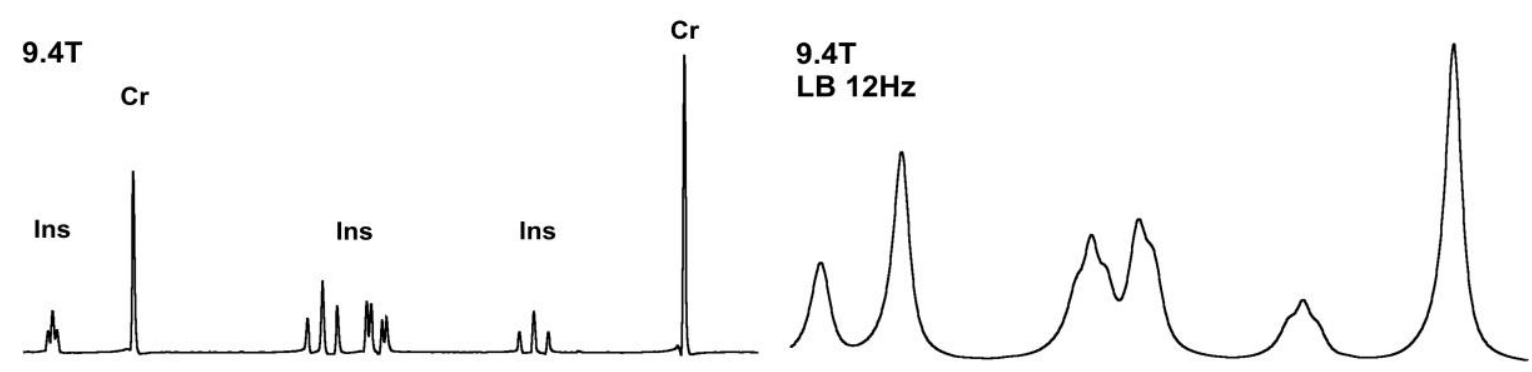

2.35T

\subsection{T}

LB $6 \mathrm{~Hz}$

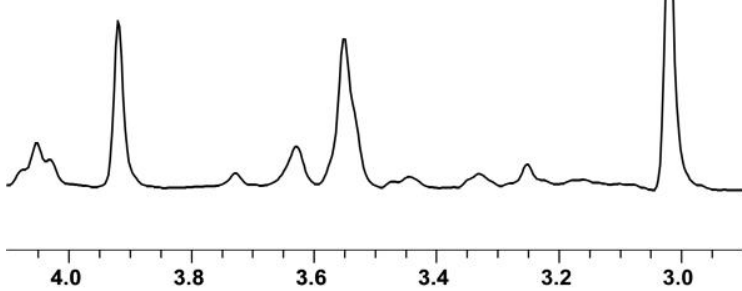

Chemical Shift / ppm

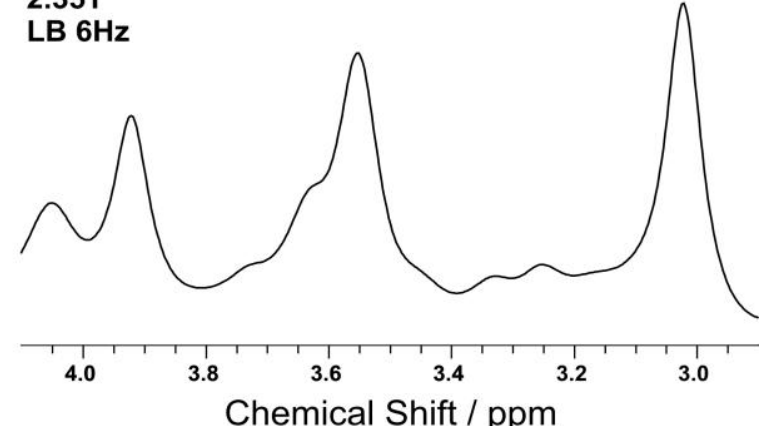

Figure 3.11: (Left) Localized proton MRS of solution with 1:1 mixture of myo-inositol (Ins) and creatine $(\mathrm{Cr})$ at $9.4 \mathrm{~T}(\mathrm{TR} / \mathrm{TE}=15000 / 10 \mathrm{~ms})$ and at $2.35 \mathrm{~T}(\mathrm{TR} / \mathrm{TE}=10000 / 10 \mathrm{~ms})$ and (right) their line broadened versions produced by multiplying exponential decay function ( $12 \mathrm{~Hz}$ and $6 \mathrm{~Hz}$ for $9.4 \mathrm{~T}$ and 2.35 $\mathrm{T}$, respectively) to render them representative of in vivo linewidth under the influence of limited resolution in each field strength. The peak intensity ratio of Ins to $\mathrm{Cr}$ at $2.35 \mathrm{~T}$ is decreased by about $50 \%$ compared to that at $9.4 \mathrm{~T}$.

\section{Glucose}

In analogy to Ins, similar reductions apply to the strongly coupled resonances of Glc, as shown in Figure 3.12.

The fully relaxed, localized proton STEAM spectrum of an aqueous solution of a 1:1 mixture of glucose and creatine, acquired at $9.4 \mathrm{~T}$, significantly differs from that at 2.35 T. The strongly coupled resonances of glucose appear in the range of $3.2-3.88 \mathrm{ppm}$ at 9.4T. Instead, in light of strong coupling effects at lower fields, the appearance of the respective resonances efficiently simplified at field strength of $2.35 \mathrm{~T}$. Namely, doubletof-doublets, centered at $3.23 \mathrm{ppm}$, disappears at low field whereas its remaining complex multiplet pattern, convert to apparent singlet at 3.43 and $3.80 \mathrm{ppm}$. In 
addition, these prominent resonances can be clearly observed in line-broadened version of spectra, which imitate the in vivo resolution.

However, these apparent glucose singlets overlap with $\mathrm{S}-\mathrm{CH}_{2}$ resonances of Tau $\alpha-\mathrm{CH}$ resonances from Glu and Gln, respectively (Gyngell et al., 1991b, Tkáć and Gruetter, 2005, Michaelis et al., 2009).

\section{Cr:Glc}

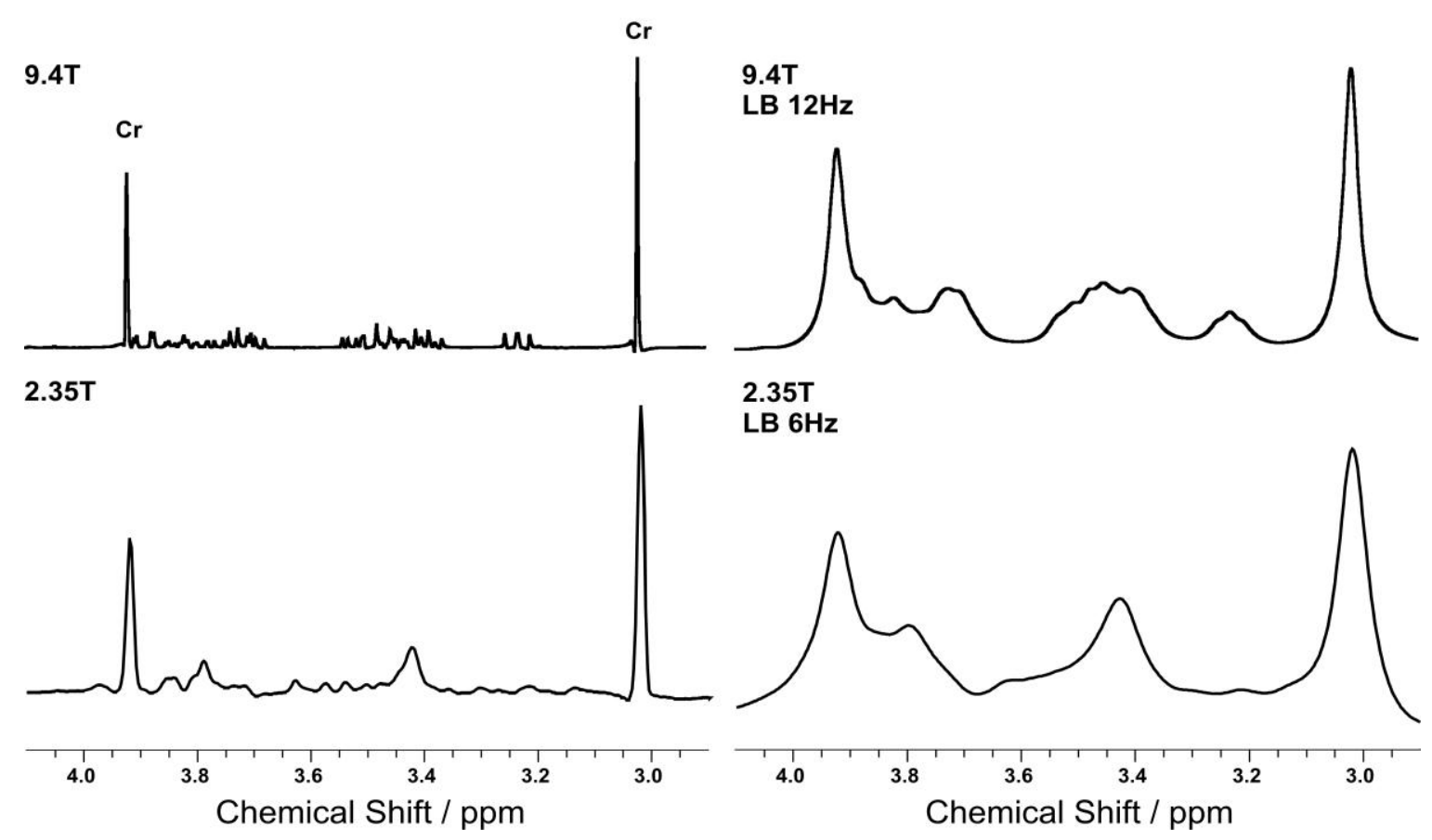

Figure 3.12: Localized proton MRS of a $1: 1$ mixture of glucose $(\mathrm{Glc})$ and creatine $(\mathrm{Cr})$ at $2.35 \mathrm{~T}(\mathrm{TR} / \mathrm{TE}=$ $10000 / 10 \mathrm{~ms})$ and at $9.4 \mathrm{~T}(\mathrm{TR} / \mathrm{TE}=15000 / 10 \mathrm{~ms})$ and their line broadened versions. The peak intensity ratio of Ins to $\mathrm{Cr}$ is clearly lower at $9.4 \mathrm{~T}$ than at $2.35 \mathrm{~T}$.

\section{Glutamate and Glutamine}

Glutamate and glutamine have similar structures and are considered to be examples for strongly coupled metabolites. Both have two methylene groups and a methine group that constitutes the strongly coupled AMNPQ spin system (Thompson and Allen, 1998, Govindaraju et al., 2000, Thompson and Allen, 2001). This strong scalar coupling interaction leads to a complex ${ }^{1} \mathrm{H}$ spectrum with distributed signal over many low intensity multiplet resonances. 
Fully relaxed proton MR spectra and their corresponding line-broadened version of a $2: 2: 1$ mixture of $\mathrm{N}$-acetylaspartate (NAA), glutamate (Glu), and glutamine (GIn) are shown in Figure 3.13. Comparing a spectrum obtained in both field strengths, illustrates the assignment problem, as a result of completely different spectral patterns in different field strengths. Strongly coupled multiplets, originating from two methylene groups of Glu, are closely grouped around 2.04 and $2.35 \mathrm{ppm}$, while those of Gln are at 2.12 and $2.45 \mathrm{ppm}$.

\section{NAA:Glu:GIn}

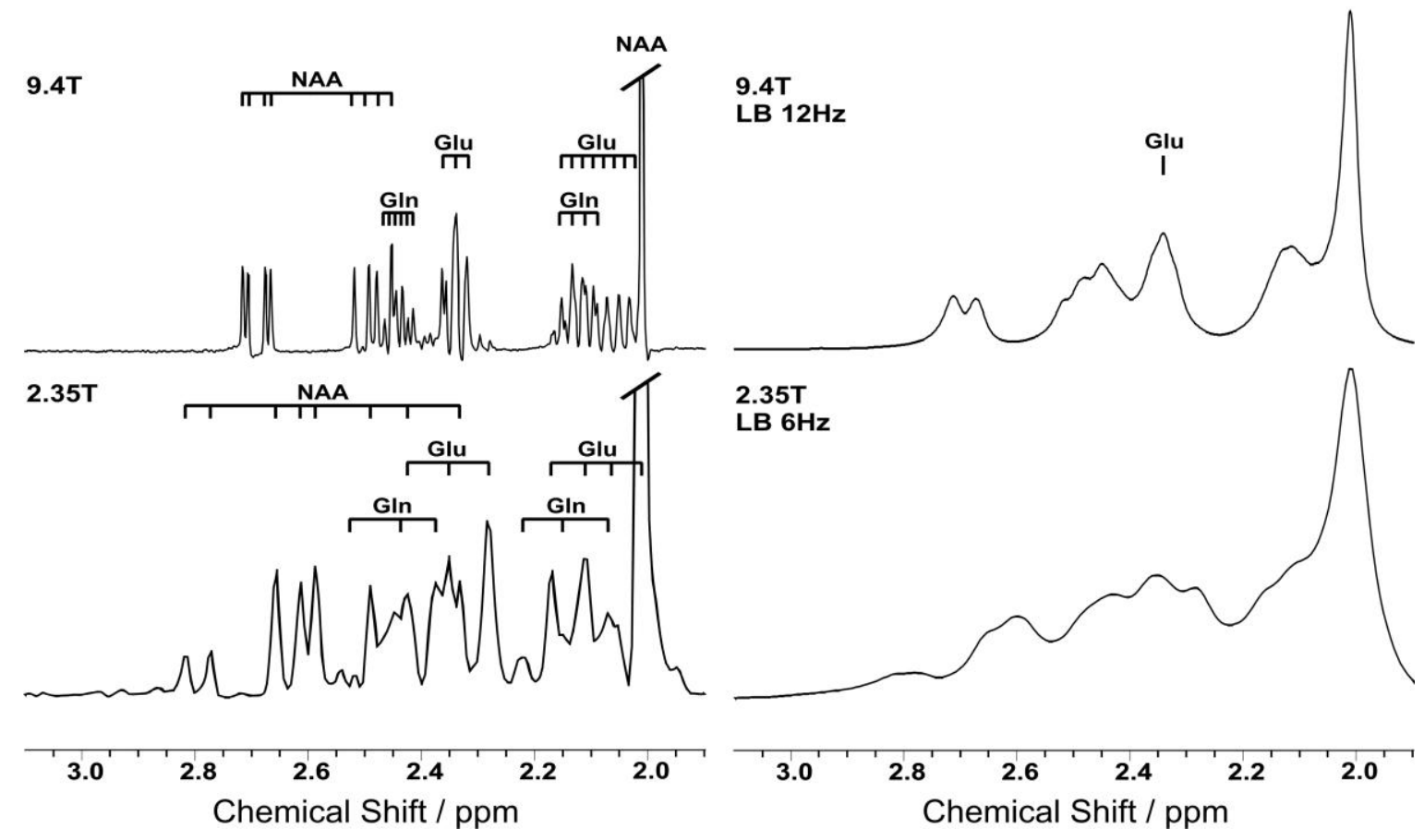

Figure 3.13: Localized proton MRS of a 2:2:1 mixture of N-acetylaspartate (NAA), glutamate (Glu), and glutamine $(\mathrm{Gln})$ at $2.35 \mathrm{~T}(\mathrm{TR} / \mathrm{TE}=10000 / 10 \mathrm{~ms})$ and at $9.4 \mathrm{~T}(\mathrm{TR} / \mathrm{TE}=15000 / 10 \mathrm{~ms})$ and their line broadened versions. The improved separation of the Glu $(2.35 \mathrm{ppm})$ and $\mathrm{Gln}(2.45 \mathrm{ppm})$ multiplets at $9.4 \mathrm{~T}$ is clearly visible.

Since the resonances of glutamate and glutamine overlap with those of NAA aspartyl moiety and GABA in this range, specific identification of their individual signal contributions in vivo is, thereby, complicated. Contrary to the myo-Ins and glucose spectral behavior at high field, improved separation of the Glu (2.35 ppm) and GIn multiplets $(2.45 \mathrm{ppm})$ at $9.4 \mathrm{~T}$ leads to a better detectability and a greatly enhanced 
quantification accuracy. On the other hand, since the separation of Glu and Gln becomes very difficult at low field, their contribution is, therefore, commonly combined (designated Glx), which improves quantification accuracy.

\section{In Vivo Experiments}

Figure 3.14 compares in vivo localized proton MR spectra of the healthy mouse brain obtained at two different field strengths. Major metabolites, e.g., tNAA, tCr, Cho, Ins, Glc, Tau, and Glu, can be readily identified. The better identification of Ins and Glc at 2.35 T, as well as Tau and Glu at 9.4 T, is demonstrated in proton MR spectra of mouse brain in vivo. The ability to acquire sufficient SNR in short measuring time is exemplified by successful outcomes of NMR spectroscopy on tiny structure, such as the hippocampus. 

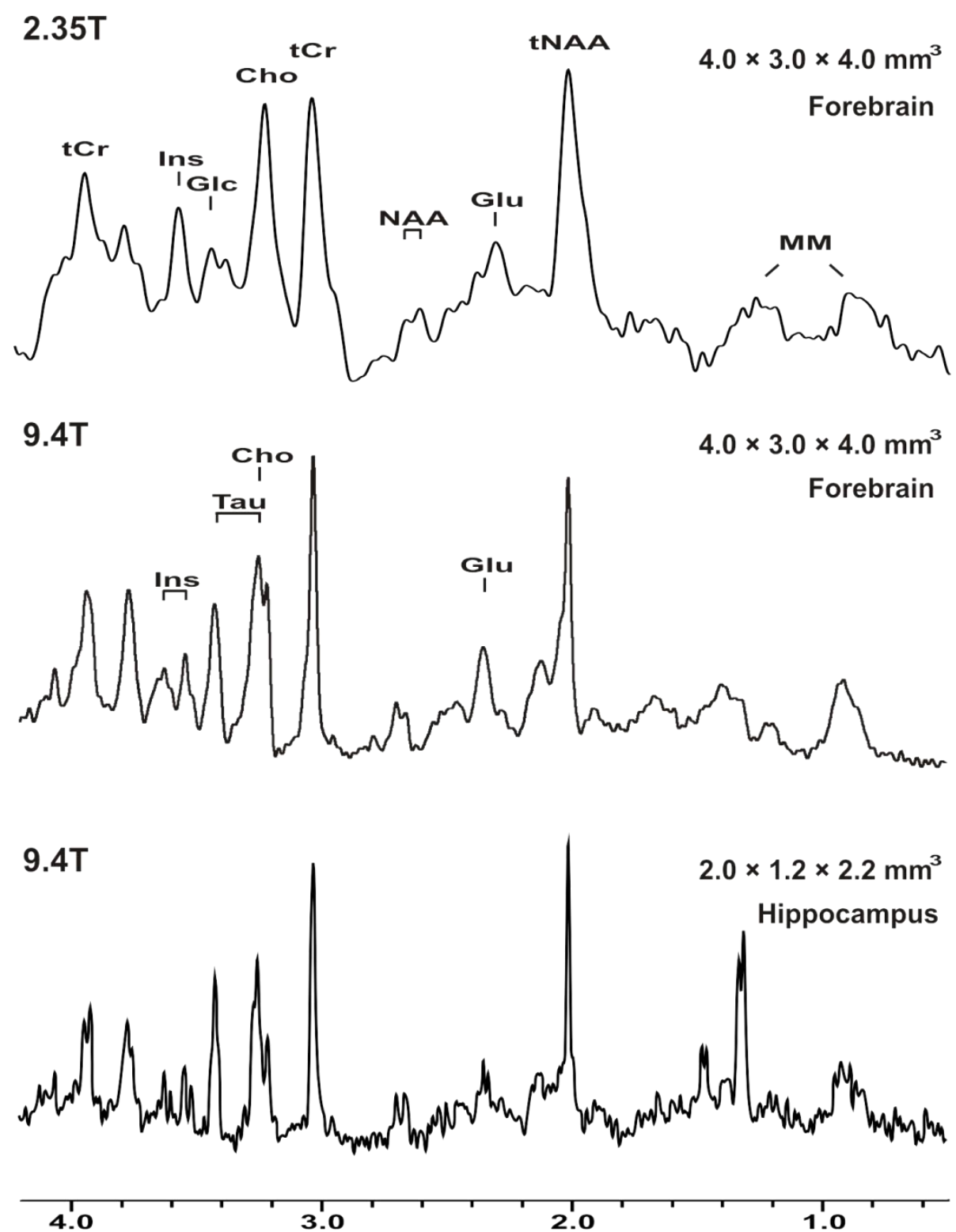

Chemical Shift / ppm

Figure 3.14: Localized proton MRS of the brain of mice (from the same strain: NMRI) in vivo at different field strengths. In comparison to (top) $2.35 \mathrm{~T}$ (STEAM, TR/TE = 6000/20 ms, 512 accumulations), (middle) 9.4 $\mathrm{T}$ (STEAM, TR/TE $=6000 / 10 \mathrm{~ms}$ ) allows for a much lower number of accumulations (32 accumulations) for the same volume-of-interest placed in the forebrain $\left(4.0 \times 3.0 \times 4.0 \mathrm{~mm}^{3}\right)$ as described by Schwarcz et al. (Schwarcz et al., 2003). The spectral resolution at $9.4 \mathrm{~T}$ is worse (which is reflected in broader linewidth) than those from smaller VOI shown in other figures because the large VOI is composed of different brain structures (e.g., gray and white matter, CSF), where a better resolution is hard to achieve. (Bottom) alternatively, high-field MRS provides sufficient SNR for a much smaller volume $\left(2.0 \times 1.2 \times 2.2 \mathrm{~mm}^{3}\right.$ covering the hippocampus) at still reduced measuring time (128 accumulations). 


\subsection{Discussion}

The improvement in local static field homogeneity, achieved by FASTMAP shimming, resulted in high and reproducible spectral resolution, which can be seen from the highly resolved spectra, obtained from different brain regions or from model solutions (Figs. 4.3 and 4.4). Consequently, efficient water suppression was attained as a result of improvement in crusher gradient performance in frequency-dependent water suppression (Moonen et al., 1992). For the selected small VOls which were studied, first and second-order terms were sufficient for compensation of field inhomogeneities.

The achieved optimal water suppression allowed a reliable detection of critical metabolite signals, despite their close proximity to the water resonance. $\mathrm{CH}_{2}$ resonance of creatine remained intact from attenuation by choosing $350 \mathrm{~Hz}$ bandwidth for CHESS pulses, while the water signal was eliminated efficiently (see Fig 3.6.). This approach guaranteed that optimal bandwidth for CHESS pulses were used for water suppression. Further, the quality of spectra (e.g., the baseline) from different regions of the mouse brain confirmed that the applied water suppression scheme is effective over a range of $T_{1}$ and $B_{1}$ values, expected for different brain regions. This is mainly achieved by judicious choice of inter-pulse delay, between each Gaussian CHESS pulse. Since the selected timing scheme was found to have an insignificant effect on the suppression performance, $\{\tau, \tau, 0.87 \tau ; \theta, \theta, 2 \theta\}$ scheme was preferred in all measurements to shorten the sequence length. Therefore, signal strength was well preserved within the localization procedure.

The $20 \mathrm{kHz}$ bandwidth of OVS RF pulses (pulse duration of $1.0 \mathrm{~ms}$ ) reduced the chemical shift displacement error of the OVS to values comparable with chemical displacement error of the VOI. STEAM localized spectra of cortical areas that are devoid of lipid contamination, justified efficacy of the incorporated OVS scheme. This was further supported by the result shown in Figure 4.4, showing data from different locations and VOI sizes. This approach allowed us substantial improvement in detection and quantification of lactate and alanine in different regions of the mouse brain.

The superior peak intensity ratios of Ins and $\mathrm{Glc}$ to $\mathrm{Cr}$ at 2.35 T refer to a better relative detectability of their respective resonances at low magnetic fields. This is due to 
the fact that their complex multiplet pattern simplifies to an apparent singlet at low field. In contrast, the larger chemical shift dispersion at 9.4 $\mathrm{T}$ yields an improved detectability of Glu and Tau resonances at high field strengths. With regard to the other major metabolites with singlet resonances, the relative detectability is fieldindependent. In general, the most important advantage of a high field is a gain in SNR. This provides access to adequately small VOls, which allows for a metabolic assessment of regional structures of mouse brain, such as the hippocampus. Alternatively, the gain in SNR may be exploited for detection and quantification of weakly represented metabolites (e.g Ala, NAAG, GABA, Asp, scyllo-Ins), which cannot be identified at 2.35 T (Schwarcz et al., 2003). 


\section{Chapter 4}

\section{Regional metabolite concentrations of mouse brain in vivo}

\subsection{Introduction}

In order to understand the structure and the function of the central nervous system, in the context of genetic information, a growing number of mutant mice are generated and investigated. Characterization of genetically modified mice is, thus, of special importance for a better understanding of pathological mechanisms, which underlie human brain disorders. To fully exploit the rapid progress in neurogenetics, the development of in vivo assessment techniques, such as noninvasive NMR technology, is highly desirable. In this regard, a number of studies using transgenic mice have demonstrated a great potential of in vivo MRI for providing detailed morphologic insights into the brain. The non-invasiveness of NMR has allowed a repeated assessment of behaving mice, which may allow an assessment of longitudinal treatment on animal models of chronic human disorders. Despite this proven advantage, there are only a limited number of published proton MRS studies on the brain of mice in vivo, probably 
due to rigorous requirements. In addition to the technical requirements described in Chapter 3, the anesthesia may be much more challenging for MRS because cerebral metabolism can be more susceptible to an altered physiological condition or to a pharmacological effect of anesthetics, in comparison to cerebral morphology. Furthermore, the small size of the brain of mice poses a specific challenge for VOls to be localized within an even smaller subdivision of the brain. In previous work, which employed the smallest VOI for mouse brain in vivo, metabolite concentrations were determined for only four different regions (Tkáć et al., 2004). To overcome these challenges, a special experimental protocol must be developed, which includes (i) selection of suitable transmit and receive coils, (ii) an anesthesia method, (iii) maintenance of body temperature, and (iv) reproducible placement of the receive coil, together with the heads of mice. In addition, $T_{1}$ and $T_{2}$ relaxation times are determined for systematic errors in metabolite quantification to be reduced because (i) the water content of the tissue in vivo, (ii) the signal attenuation between the tissue in vivo and the metabolite model solution in vitro, and (iii) the partial volume effect of the cerebrospinal fluid, may influence the quantification.

High reproducibility is essential to unveil subtle variation of neurochemical profiles in the longitudinal investigations of physiological or pathophysiological processes. Intra-individual variability in the data from single-voxel localized ${ }^{1} \mathrm{H}$ MRS examinations are generally associated with various factors, including biological variability, instrumental instability, animal and the VOI positioning, operator variability and spectral analysis methods. Several studies have been conducted to demonstrate the degree of reproducibility in ${ }^{1} \mathrm{H}$ MRS of the human brain in vivo, either using different localization sequences, locations and timing parameters (Geurts et al., 2004, Inglese et al., 2006) or different field strengths of 1.5 T (Alger et al., 1993, Brooks et al., 1999, Geurts et al., 2004, Hammen et al., 2005, Helms, 2000, Kreis et al., 2005, Schirmer and Auer, 2000), 2.0 T (Choi and Frahm, 1999, Michaelis et al., 1993b), 3.0 T (Milne et al., 2009, Hancu et al., 2005), 4.0 T (Bartha et al., 2000, Bartha, 2007), and 7.0 T (Tkáć et al., 2009, Tkáć et al., 2002). So far, however, only a few studies have experimentally investigated the intra- and inter-individual variability in rodent brain in vivo (Pfeuffer et al., 1999, Öz et al., 2010, Hong et al., 2011b). Therefore, in the present study, Intra- and 
inter-individual reproducibility of $M R$ spectroscopic acquisition protocol and corresponding LCModel data analysis were assessed, based on the variability of metabolite concentrations in healthy mouse brain at 9.4 T using a localization sequence, based on stimulated echo acquisition mode [STEAM]. Absolute metabolite concentrations (mean $\pm S D$ ), the coefficients of variation (CV) and Crame'r-Rao lower bounds from intra-individual studies were compared to those from inter-individual studies, obtained under similar experimental conditions.

\subsection{Materials and Methods}

\section{Animal preparation}

All studies were performed in accordance with German animal protection laws and approved by the responsible governmental authority. The experiments were performed on nine adult healthy female NMRI mice (body weight in the range 34-38 g). Anesthesia was induced, using a chamber pervaded with $5 \%$ isoflurane in oxygen. The mice were intubated with a purpose-built polyethylene endotracheal tube and artificially ventilated afterward. Anesthesia was maintained with 1 to $1.5 \%$ isoflurane in a 1:1.5 mixture of oxygen and ambient air. The animals were then placed in a prone position on a home-built palate holder, equipped with an adjustable nose cone and ear bars. This sophisticated stereotaxic animal cradle ensured stability and reproducibility of the experimental setup by properly immobilizing the animal's skull during measurements. Body temperature was maintained constant around $37^{\circ} \mathrm{C}$ using heated water blankets, placed over the animal and connected to a temperature-controlled circulating water bath. A rectal thermosensor was utilized for temperature verification throughout the experiment. Respiration was monitored by a pressure transducer, which was fixed to the animal's chest. Details of the experimental setup used for in vivo MRI/MRS of mouse brain at 9.4 $\mathrm{T}$ are shown in Figure 4.1. 


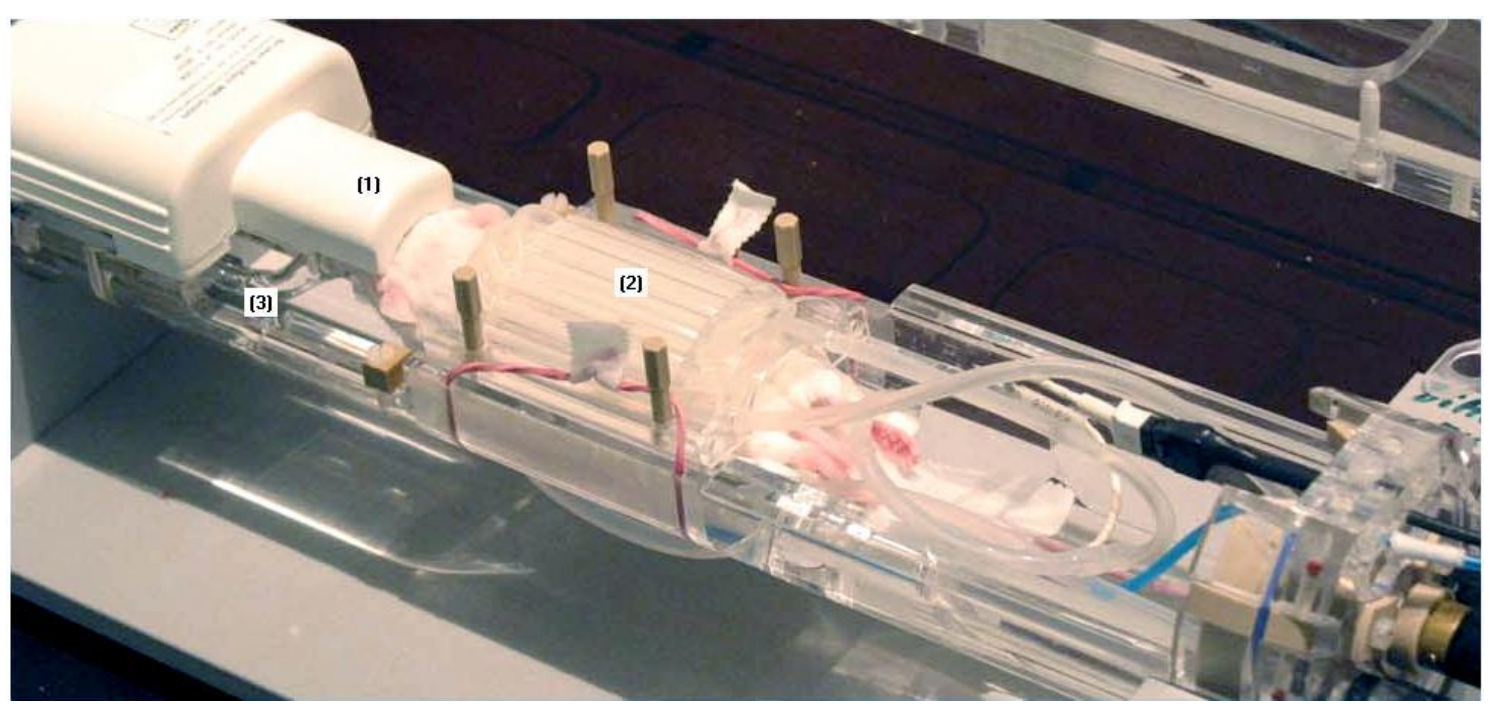

Figure 4.1: Experimental setup for localized ${ }^{1} \mathrm{H}$ MR spectroscopy of mouse brain at 9.4 T. Radiofrequency excitation and signal reception were accomplished by a quadrature birdcage coil (not shown) and a quadrature mouse brain surface coil (1), respectively. The body temperature was maintained constant using a heated water blanket (2) positioned around the body. The animals were anesthetized and intubated with a polyethylene endotracheal tube (3) and artificially ventilated. Their heads firmly fixed by means of a home-made stereotaxic palate holder with an adjustable nose cone. Source: from Michaelis et al., (Michaelis et al., 2009). Courtesy of Dr. Roland Tammer (rtammer@gwdg.de).

\section{Proton MR Spectroscopy}

The experiments were performed at $400 \mathrm{MHz}$ on a $9.4 \mathrm{~T}$ horizontal superconducting magnet with $30 \mathrm{~cm}$ bore size (Bruker Biospec Avance II 94/30; Bruker, Karlsruhe, Germany) and equipped with a $12 \mathrm{~cm}$ inner diameter self-shielded gradient coil insert (Resonance Research Inc, Billerica, MA, USA), capable of supplying up to $400 \mathrm{mTm}^{-1}$ in $80 \mu$ s rise time. RF amplifier with $2 \mathrm{~kW}$ peak power was used because preliminary study has shown that it allows shorter slice-selection RF pulse, which consequently reduces the extent of the chemical shift displacement error, in comparison with standard $1 \mathrm{~kW}$ RF power amplifier.

A commercially available, $72 \mathrm{~mm}$ quadrature volume coil was used for excitation and a quadrature mouse brain surface coil (Bruker, Karlsruhe, Germany) was used for signal detection. For the measurement of ${ }^{1} \mathrm{H}$ NMR spectra from forebrain regions (e.g., cerebral cortex, striatum, thalamus, and hippocampus), the surface coil was located 
upon the head of the mouse, as shown in Fig. 4.1. For spectra from hindbrain regions (e.g., cerebellum and brainstem), it was relocated caudally to maximize signal reception. The system was interfaced to a Linux operating system, running Topspin 1.5 and Para Vision 4.0 imaging software (Bruker Biospin, Germany).

Axial and sagittal $\mathrm{T}_{2}$-weighted multi-slice RARE (Rapid Acquisition with Relaxation Enhancement) (Hennig et al., 1986) images were obtained to carefully select VOI positions for localized proton MRS. The timing parameters used to collect the MRI data were TR/TE $=4200 / 14 \mathrm{~ms}$, RARE factor $=8$, number of averages $=2$, slice thickness $=0.5$ $\mathrm{mm}$, field of view $=26 \times 26 \mathrm{~mm}$, matrix size $=128 \times 128$. Figure 4.2 shows typical placement of the volumes-of-interest (VOIs) for ${ }^{1} \mathrm{H}$-MRS of ten different brain regions, with sizes ranging from 4.2 to $15.5 \mu \mathrm{l}$. Since no lateralisation of metabolite levels in normal adult brain was observed in previous studies (Choi and Frahm, 1999, NagaePoetscher et al., 2004), VOls were unilaterally localized in the left hemisphere for the cerebral cortex (5.6 $\mu \mathrm{L}$, "Lateral Cortex"), callosal fibres $(4.2 \mu \mathrm{L})$, striatum (11 $\mu \mathrm{L})$, thalamus (9.2 $\mu \mathrm{L}$, "Lateral Thalamus") and hippocampus (4.6 $\mu \mathrm{L})$. In addition, VOIs are selected for the medial part of the frontal cortex (6.75 $\mu \mathrm{L}$, "Medial Cortex"), corpus callosum (4.76 $\mu \mathrm{L})$, thalamus (15.52 $\mu \mathrm{L}$, "Medial Thalamus"), brainstem (8.25 $\mu \mathrm{L})$ and cerebellum $(6.12 \mu \mathrm{L})$. Lateral VOIs were placed in the left hemisphere of the mouse brain. As the "Lateral Cortex" and the "Medial Cortex" were close to the scalp, these VOIs were carefully placed to exclude the fat tissue of the scalp. For the corpus callosum and the brainstem, the VOI was tilted around the $\mathrm{X}$ axis in order to achieve a better localization. For the "Lateral Cortex" and the callosal fibres, a tilt around the $\mathrm{X}$ axis, along with a tilt around the $Z$, was applied. This double-oblique localization was necessary for the "Lateral Cortex" to exclude the neighbouring white matter, whereas it enabled an optimal exclusion of the grey matter from the "callosal fibres". As far as the brainstem was concerned, the VOI included both the pons and the medulla oblongata. With regard to the cerebellum, the VOI covered its medial portion, including the vermis. To minimize the inter-individual variability in the placement of these VOIs, a number of anatomical structures were used as landmarks (e.g. the midsagittal plane, the lateral wall of the anterior horn of the lateral ventricle, the rostral border of the dorsal hippocampal formation, and the border between the hypothalamus and the basal cistern). 
The oblique, rather than orthogonal localization, may lead to an inadequate performance which causes a variation in concentration, although it offers a potential advantage of accurate localization with optimal SNR, while minimizing partial volume effect (i.e., contamination of CSF or other brain tissue). Optimization of VOI can facilitate selection of any desired volume. Therefore, the performance of oblique localization was investigated. To ascertain the possible effect of choosing oblique VOI on the corresponding localization performance, VOI of $27 \mu \mathrm{L}$ were measured with different angles and directions, with respect to the main coordinate axes $x, y, z$.

Table 4.1. Concentration of creatine in 6 different oblique VOI determined by LCModel normalized to $\mathrm{Cr}$ obtained in orthogonal VOI (angle $=0$ )

\begin{tabular}{lcl}
\hline \hline Axis & Angle & $\begin{array}{l}\text { Cr } \\
\text { (normalized) }\end{array}$ \\
\hline & 0 & $100.0 \pm 2.1$ \\
$\mathbf{X}$ & +45 & 95.7 \\
$\mathbf{X}$ & -45 & 102.0 \\
$\mathbf{Y}$ & +45 & 99.0 \\
$\mathbf{Y}$ & -45 & 94.9 \\
$\mathbf{Z}$ & +45 & 96.8 \\
$\mathbf{Z}$ & -45 & 98.3 \\
\hline
\end{tabular}

The absolute creatine concentration obtained from an orthogonal VOI in the center of a $3-\mathrm{cm}$ diameter glass sphere (filled with distilled water containing $50 \mathrm{mM}$ creatine) was normalized to 100 . The quantitative analysis of the determined concentrations, summarized in Table 4.1, revealed that variation of concentration remained within experiment reproducibility, therefore indicating an adequate localization performance. 


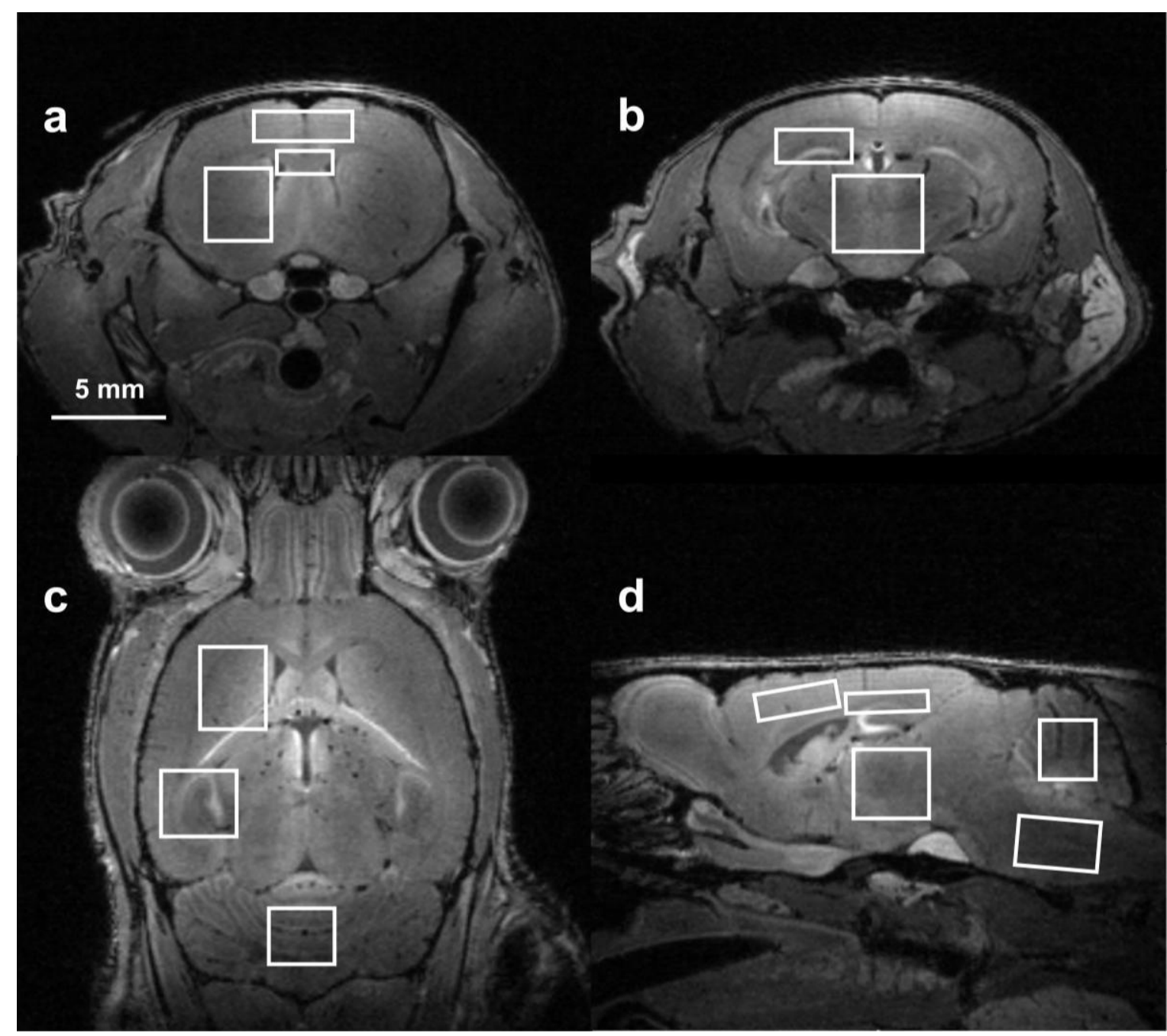

Figure 4.2: $T_{1}$-weighted 3D multi-slice RARE images $(T R / T E=4200 / 14 \mathrm{~ms}$, RARE factor $=8$, matrix size $=$ $128 \times 128)$ of the mouse brain $(\mathrm{NMRI})$ representing typical placement of volumes of interest (VOIs) selected for ${ }^{1} \mathrm{H}$-magnetic resonance spectroscopy of the Medial Cortex (a), corpus callosum (a), striatum $(a, c)$, hippocampus $(b, c)$, Medial Thalamus (b), Cerebellum (c,d), Lateral Cortex (d), callosal fibers (d), Lateral Thalamus (d), brainstem (d).

Two sequential fully relaxed short echo-time proton MR spectra (STEAM, $\mathrm{TR} / \mathrm{TE} / \mathrm{TM}=6000 / 10 / 10 \mathrm{~ms}, 128$ averages) were obtained from the corresponding brain regions. For each measurement, spectral width was set to $5000 \mathrm{~Hz}$ and 4096 data points were acquired. The unsuppressed water signal, measured from the same VOI, was exploited as an internal reference for quantification (Barker et al., 1993, Soher et al., 1996). In order to alleviate unwanted effect of frequency drifting or shim instability, during acquisition on spectral line width, the number of accumulations was restricted to 128 for each recording. Mean spectra were created from acquired individual in vivo spectra, consisting of $2 \times 128$ for each animal, yielding satisfactory signal-to-noise ratio 
(SNR) as well as reliability of metabolite detection and quantification. Adjustment of all first and second order shim terms were performed automatically for each VOI using FASTMAP (Gruetter, 1993) method. Optimization of field homogeneity (shimming) routinely resulted in unsuppressed water signal line widths (full-width at half-maximum [FWHM]) of 11-14 Hz and $9 \mathrm{~Hz}$ for metabolites in vivo (measured on the $\mathrm{Cr} / \mathrm{PCr} \mathrm{CH}_{3}$ peak at 3.0 ppm). Optimized CHESS-type (Frahm et al., 1990, Ernst and Hennig, 1995) method was employed for water suppression and to improve quality of localization, interleaved with outer volume saturation. Both preceded the STEAM sequence. The parameters used for MRS data acquisition and FASTMAP shimming are a consequence of method optimization, described in the third chapter, and therefore, are only briefly outlined here.

\section{Quantification of metabolites}

The resulting in vivo spectra were analysed in the frequency domain by using LCModel 6.2-0 (Linear Combination of Model spectra of metabolite solutions in vitro). This user-independent and fully automated fitting routine estimates absolute metabolite concentrations by incorporation of a priori knowledge, which are usually referred to as a "basis set" into the data evaluation (Provencher, 1993). Basis spectra are obtained from individual metabolites at known concentration in aqueous solution, under identical experimental conditions as those in the in vivo acquisition. LCModel obtains an optimal fit to the in vivo spectra by finding the smoothest line shape and baseline, consistent with the data, using a constrained regularisation algorithm (Provencher, 2001). The method takes advantage of full spectral features of each individual basis spectrum for evaluation, rather than individual resonances. Therefore, it allows discrimination between metabolites with overlapping signals. Raw data obtained from measurement was directly supplied to LCModel. Adjustment of phases, determination of referencing shift, estimation of baseline and the uncertainties in the concentrations (Crame'r-Rao lower bounds), as well as eddy current correction, are automatically accomplished.

Unsuppressed water signal, measured from the same VOI under identical conditions, was used for metabolite quantification, assuming a constant brain water 
concentration of $43.7 \mathrm{~mol} / \mathrm{L}$ (Schwarcz et al., 2001, Schwarcz et al., 2003). Hence, concentrations are expressed as $\mathrm{mM}$, i.e., millimoles of metabolite per volume of tissue [mmol/ (litre VOI)]. Using water referencing inherently overcome problems arising from different coil loadings, different voxel sizes in addition to regional inhomogeneities in surface coil sensitivity profile. Therefore, it allows feasibility of reliable and accurate absolute quantification with use of surface coil (Kreis et al., 1993a, Danielsen and Henriksen, 1994, Kreis, 1997, Michaelis et al., 1999, Pfeuffer et al., 2004, Jansen et al., 2006).

Spectra of 16 brain metabolites were included in LCModel basis-set: alanine (Ala), aspartate (Asp), creatine $(\mathrm{Cr})$, phosphocreatine (PCr), $\gamma$-aminobutyric acid (GABA), glucose (Glc), glutamate (Glu), glutamine (Gln), glycerophosphorylcholine (GPC), phosphorylcholine (PCh), myo-inositol (Ins), lactate (Lac), $\mathrm{N}$-acetylaspartate (NAA), $\mathrm{N}$ acetylaspartylglutamate (NAAG), scyllo-inositol (scyllo-Ins) and taurine (Tau). Furthermore, sum concentrations were evaluated for those metabolites with a strong cross-correlation, originating from similarity in their structure and spectral patterns. Therefore, the sums of NAA + NAAG, GPC + PCho (total choline-containing compounds), $\mathrm{PCr}+\mathrm{Cr}$ (total creatine) and Glu $+\mathrm{Gln}$ were reported. LCModel analysis was performed in the chemical shift range of $0.5-4.2 \mathrm{ppm}$.

Crame'r-Rao lower bounds (CRLB) of LCModel analysis were used to assess accuracy and reliability of the fitting, which is regarded as the metabolites concentration estimated errors and reflect the estimated standard deviation (\%SD) of the metabolite fit (Cavassila et al., 2001, Kreis, 2004, Helms, 2008). CRLB higher than 50\% was considered as exclusion criterion for metabolite evaluation. Thus, only concentrations with CRLB below 50\% were taken into account in the analysis. In addition, the residuals (original spectrum minus fitted spectrum) for each spectrum were visually inspected for presence of spurious artifacts to check adequate convergence of the peak fitting. Other rejection criteria, such as poor SNR $(<6)$, existence of strong baseline distortions and line widths (full-width at half-maximum peak height, FWHH), which exceeded the expectations limits, were applied (Jansen et al., 2006, Poullet et al., 2008).

To account for the potentially confounding effect of fast relaxing macromolecules and mobile lipids on metabolites quantitation in short-echo ${ }^{1} \mathrm{H} \mathrm{MR}$ 
spectroscopy, their corresponding simulated signal were incorporated into the basis set of LCModel (Behar and Ogino, 1993, Michaelis et al., 1993b). Regularized spline baseline modelling of LCModel imitates most physiological characteristics of short- $T_{1}$ macromolecules spectrum (Provencher, 2001). Hofmann et al evaluated the influence of using an experimentally determined macromolecule baseline as prior knowledge (Hofmann et al., 1999, Hofmann et al., 2002) on metabolite concentration estimation and compared the results with conventional LCModel fit. For that reason, in this study, no further attempt was made to experimentally include the physiological macromolecular pattern in the basis set of LCModel. However, to incorporate measured macromolecule signals, one can acquire In vivo metabolite-nulled spectra which, allow reliable quantification of macromolecules (Pfeuffer et al., 1999, Auer et al., 2001, Seeger et al., 2003).

\section{Measurement of the basis set}

Model spectra were recorded from aqueous model solution of alanine (Ala), aspartate (Asp), phosphocholine (PCh), glycero-PCh (GPC), creatine (Cr), phosphocreatine (PCr), $\gamma$-aminobutyric acid (GABA), glucose $(\mathrm{Glc})$, glutamine (Gln), glutamate (Glu), glutathione (GSH), myo-inositol (ml), Scyllo-inositol (sl), lactate (Lac), Nacetylacetate (NAA), N-acetylaspartylglutamate (NAAG), taurine (Tau), phosphorylethanolamine (PE), ascorbic acid (Asc), glycine (Gly), acetate (Ace), threonine (Thr), propylenglycol (Pgc)and ethanol (Eth), according to Provencher (S. Provencher, LCModel \& LCMgui User’ s Manual, http://s-provencher.com/pages/lcmodel.shtml).

Spectrum of scyllo-inositol was simulated by shifting the singlet of glycine from 3.55 to $3.35 \mathrm{ppm}$. All chemicals were purchased from Sigma-Aldrich and Fluka. Metabolites were dissolved separately in an aqueous phosphate buffer $\left(72 \mathrm{mM} \mathrm{K}_{2} \mathrm{HPO}_{4}\right.$, $28 \mathrm{mM} \mathrm{KH}_{2} \mathrm{PO}_{4}$ ), containing 3-(trimethylsilyl)-propanesulfonic acid sodium salt (DSS), as chemical shift reference ( $0 \mathrm{ppm})$ and $\mathrm{pH}$ was subsequently adjusted to 7.20. $200 \mathrm{mM}$ sodium formate was added for automatically phasing and scaling of model spectra by using its singlet at $8.44 \mathrm{ppm}$. For choline containing compounds, the phosphate buffer 
was replaced by $100 \mathrm{mM}$ potassium chloride $(\mathrm{KCl})$. Metabolite signal of the model solution was obtained by measuring a VOI size of $3-4 \mathrm{~cm}^{3}$ centrally located in a spherical phantom of $11 \mathrm{ml}$. The scaled spectra from all solutions are presented in Figure 4.3.

A $72 \mathrm{~mm}$ quadrature birdcage coil was used for both signal excitation and reception. Fully relaxed basis sets were acquired with sufficiently long repetition time $(T R=15 \mathrm{~s})$, obviating the need for in vitro $\mathrm{T}_{1}$ relaxation correction but with otherwise identical experimental conditions (STEAM, TE/TM=10/10 ms) to those of the in vivo measurements.

Due to the fact that "Auto-Phasing" failed to correctly phase the acquired Basis Spectra, accurate values for zero- and first-order phase correction, $\varphi_{0}$ and $\varphi_{1}$, were manually determined for each metabolite in the Basis Set.

For precise and consistent quantification, concentrations of GPC, NAAG and all other prominent singlets were individually calibrated to the $\mathrm{CH}_{3}$ resonance of creatine peak. For this purpose, it is necessary to consider how many protons effectively contribute to the singlet. This method resolves the ambiguity in determining concentration, which arises from the preparation of model solution, i.e., (i) uncertainty in the molecular weight of GPC because GPC in vitro needs cadmium chloride adduct, and (ii) inaccurate water content value for hydrated compounds, e.g., PCr. It should be noted that the method exploits the fact that the concentration of the dissolved anhydrous creatine can be precisely determined. 

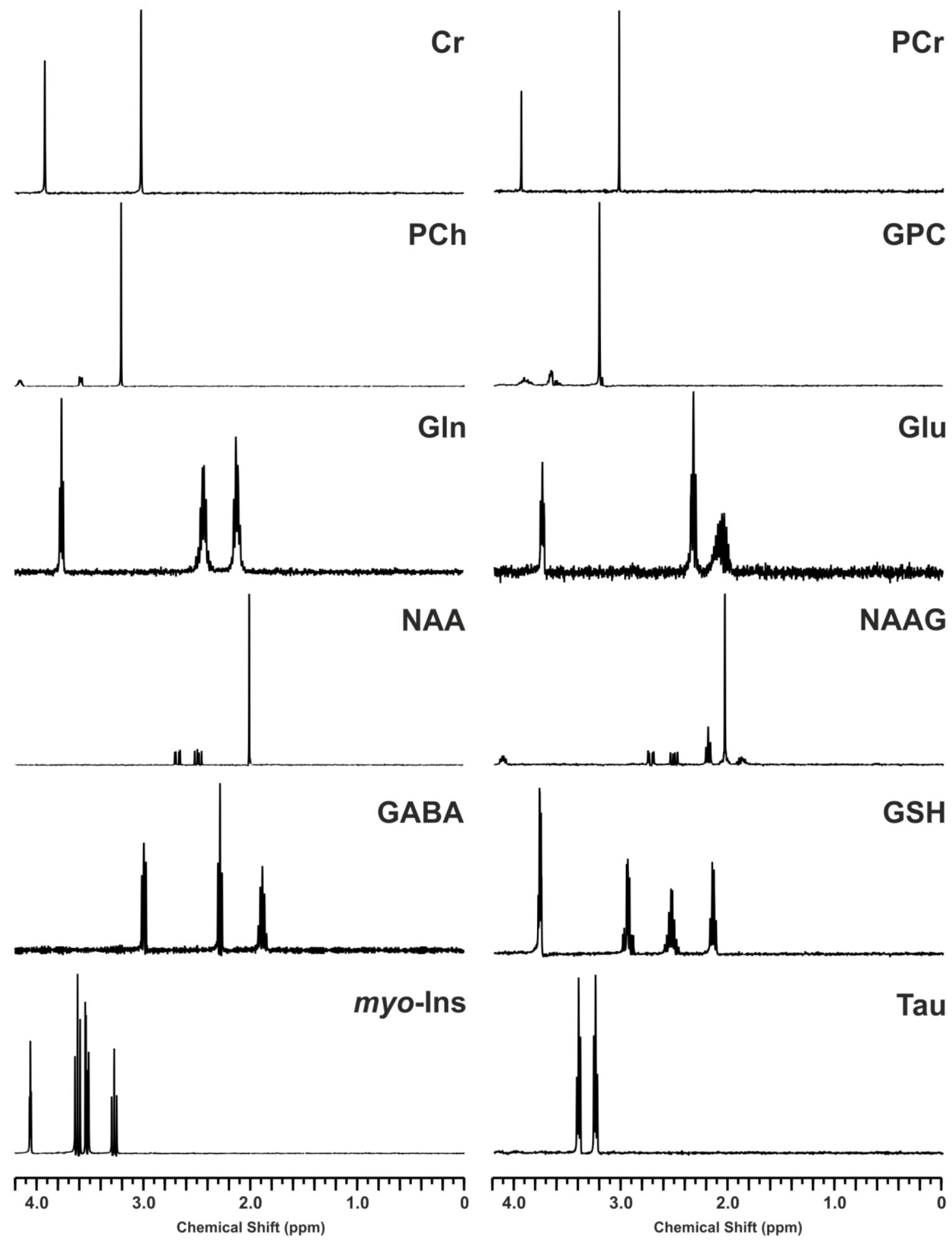

Figure 4.3: Representative short-echo basis spectra for LCModel, measured with the STEAM sequence from model solutions ( $\mathrm{pH}$ 7.20). (For details and abbreviations, see text.) 

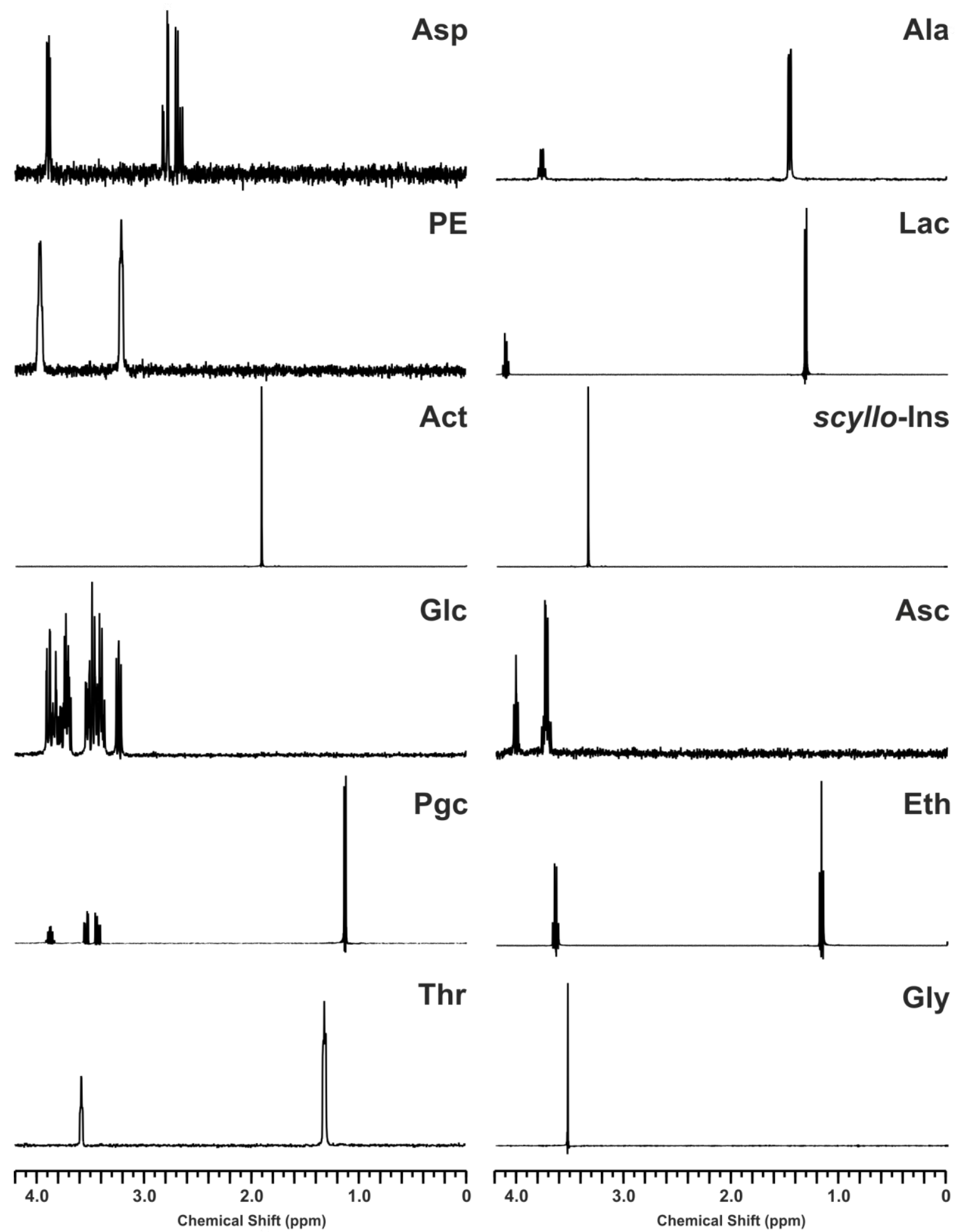

Figure 4.3: Continued. 


\section{Assessment of the $T_{1}$ and $T_{2}$ relaxations and their impact on metabolite quantification}

Nevertheless, a precise estimation of water content is essential for absolute quantification of metabolite concentration by LCModel because the signal of metabolites must be calibrated to that of water. Metabolite signal is supposed to be originated mainly from brain tissue, while a localized volume-of-interest may contain, not only brain tissue, but also cerebrospinal fluid (CSF). Thus it has been proposed that compartmentation information has to be determined on the basis of image segmentation (C.W. Brooks et al., 1999, Schuff et al., 2001, Horská et al., 2002) or spectroscopy pulse sequence (Hennig et al., 1992). Further, $T_{1}$ and $T_{2}$ attenuation of water signal may lead to potential errors for accurate absolute metabolite quantification. The water content of the localized volume-of-interest may be calculated from $T_{1}$ relaxation time of the brain tissue.

The water $T_{1}$ relaxation time was determined in all the aforementioned brain structures. A series of STEAM experiments, without water suppression, and with varying repletion times of 580, 700, 800, 1000, 1200, 1500, 1700, 2000, 3000, 5000, 6000, 7000, $8000,10000,12000$ and $15000 \mathrm{~ms}$ were obtained. To yield apparent $T_{1}$, the water signals were fitted to mono-exponential function, according to Equations 4.2 and 4.4. Other parameters of the applied STEAM sequence were kept identical: TE $=10 \mathrm{msec}$, mixing time $(T M)=10 \mathrm{msec}$ and 8 accumulations.

For $T_{2}$ measurement, peak heights of unsuppressed water FIDs, obtained from aforementioned series of STEAM experiments, were fitted to monoexponential decay functions. This procedure obviates problems arising from variability of linewidth, due to different quality of achieved shimming.

\section{Partial volume effect}

Spectroscopic $T_{2}$ method was exploited to determine the contributions from cerebrospinal fluid (CSF) within the selected volumes. This has been accomplished for all 
locations - even for the relatively small voxels used in this study. Series of the unsuppressed water signal in proton MR spectra were acquired at 12 different echo times (10 ms - $1000 \mathrm{~ms}$ ) and water signal intensities from time-domain data (FIDs) were fitted to a bi-exponential model function (Ernst et al., 1993, Kreis et al., 1993b), according to the following equation:

$$
S=S_{\mathrm{TE}=0, \mathrm{BW}} \cdot \exp \left(-\frac{T E}{T_{2, \mathrm{BW}}}\right)+S_{\mathrm{TE}=0, \mathrm{CSF}} \cdot \exp \left(-\frac{T E}{T_{2, \mathrm{CSF}}}\right)
$$

$\mathrm{S}_{\mathrm{TE}=0, \mathrm{BW}}$ and $\mathrm{S}_{\mathrm{TE}=0, \mathrm{CSF}}$ are relaxation-corrected signal amplitudes for brain water (BW) and cerebrospinal fluid (CSF), respectively. Standard least squares fitting procedure was performed with curve-fitting tool of Matlab (Version 7.1; The MathWorks, Inc., Natick, MA, USA). Fractional water content of the selected voxel with mixed proportions of CSF and brain matter (partial volume averaging) was calculated from the component analysis, considering the fact that longer $T_{2}$ component ascribed to CSF ( $T_{2, \text { CSF }}$ ).

A survey of potential partial volume contributions from the cerebrospinal fluid (CSF) within the investigated VOls was achieved by analyzing least squares fit of TE series data points to a bi-exponential and mono-exponential model functions, according to Equations 4.1 and 4.2. The mono-exponential fitting always resulted in better fit and the long $T_{2}$ component of the bi-exponential decay, assigned to CSF, was not detected. These findings indicate that localization was optimal and CSF contribution to the volumes can be considered to be negligible.

\section{Signal attenuation}

Water attenuation can be regarded as representative of potential error for accurate absolute metabolite quantification, whereas unsuppressed water signal served as internal reference. To examine influence of relaxation attenuation, transverse relaxation times $\left(T_{2}\right)$ of water, as well as its longitudinal relaxation $\left(T_{1}\right)$, were determined in different regions.

Equation 4.2 describes attenuation of the NMR-visible water signal as a result of relaxation effects in STEAM. 


$$
S(T R, T E, T M)=S_{0} \cdot f_{\mathrm{T} 2} \cdot f_{\mathrm{T} 1}
$$

where

$$
\begin{gathered}
f_{\mathrm{T} 2}=\exp \left(-\frac{T E}{T_{2}}\right) \\
f_{\mathrm{T} 1}=\exp \left(-\frac{T M}{T_{1}}\right)\left[1-\exp \left(-\frac{T R}{T_{1}}\right)\right]
\end{gathered}
$$

$f_{\mathrm{T} 2}$ and $f_{\mathrm{T} 1}$ indicate corresponding relaxation losses as a consequence of $\mathrm{T}_{2}$ and $T_{1}$, involving those throughout the middle interval (TM), where the delay is within the second and third pulses of the STEAM sequence.

LCModel's control parameter atth2o, which is basically $f_{\mathrm{T} 2} \times f_{\mathrm{T} 1}$, takes care of correction for relaxation attenuation of water signals. Owing to the sufficiently short echo time ( $T E=10 \mathrm{~ms}$ ) and long repetition time ( $\mathrm{TR}=6000 \mathrm{~ms}$ ) used in all in vivo measurements, this attenuation factor was kept constant (ATTH2O=0.7), although, the impact of regional variability of the relaxation on this factor was evaluated.

It is assumed that model spectra were acquired with identical parameters as in vivo data and that metabolites possess different relaxivity in in vitro and in vivo conditions. As a consequence, pertinent correction factors, induced by residual $\mathrm{T}_{2}$ relaxation effects $\left(f_{\Delta T_{2}}\right)$ and differential $T_{1}$ saturation $\left(f_{\Delta T_{1}}\right)$, can be estimated for each metabolite from:

$$
\begin{gathered}
f_{\Delta T_{1}}=\frac{1-\exp \left(-T R / T_{1}^{\text {in vitro }}\right)}{1-\exp \left(-T R / T_{1}^{\text {in vivo }}\right)} \\
f_{\Delta T_{2}}=\frac{\exp \left(-T E / T_{2}^{\text {in vivo }}\right)}{\exp \left(-T E / T_{2}^{\text {in vitro }}\right)}
\end{gathered}
$$

Two major metabolites, glutamate as a strongly coupled one and creatine as an uncoupled one, were chosen to exemplify possible correction factors, accounting for the differential $\mathrm{T}_{2}$ attenuation between in vivo and in vitro conditions. In the light of correction of concentrations for residual $T_{2}$ relaxation effects, the $T_{2}$-values were taken from literature (Xin et al., 2008) and regional variations in $T_{2}$ relaxation times of 
metabolites were disregarded. Diffusion-induced signal attenuation, brought about by incorporation of crusher gradient into the TE/2 intervals, was disregarded due to its minor contribution and steady presence in employed STEAM sequence (de Graaf et al., 2001).

To evaluate the amount of signal attenuation due to relaxation, quantitative localized $T_{2}$ measurements were pursued in vivo for all structures used in this study. $T_{2}$ relaxation times of tissue water were obtained by mono-exponential fitting. Table 4.2 summarizes the $T_{2}$ relaxation times (mean values and SDs) of water in various mouse brain regions measured in vivo at $9.4 \mathrm{~T}$. The results from the analysis, as presented in Table 4.2, showed regional differences of brain water $T_{2}$ relaxation, of which, highest values were observed in the cortex, while those of brain stem and cerebellum were at the lowest.

Table 4.2 Mean and SD of tissue water proton $T_{2}$ relaxation times ( $\mathrm{ms}$ ) determined in different region of the normal mouse brain in Vivo

\begin{tabular}{|c|c|c|c|c|c|c|c|c|c|c|}
\hline & \multicolumn{10}{|c|}{ Structure } \\
\hline & $\overline{m c x}$ & lcx & $\overline{\mathrm{CC}}$ & cf & st & mth & lth & $\overline{\mathrm{hc}}$ & $\mathrm{cb}$ & bs \\
\hline & $n=8$ & $n=7$ & $n=9$ & $n=8$ & $n=8$ & $\mathrm{n}=9$ & $n=9$ & $\mathrm{n}=9$ & $n=8$ & $n=5$ \\
\hline $\mathrm{T}_{2}(\mathrm{~ms})$ & 36.6 & 36.0 & 34.9 & 35.0 & 35.4 & 35.3 & 33.7 & 36.0 & 32.8 & 33.0 \\
\hline $\mathrm{SD}(\mathrm{ms})$ & 0.7 & 1.0 & 1.6 & 1.5 & 0.7 & 0.5 & 0.5 & 1.1 & 0.5 & 0.7 \\
\hline
\end{tabular}

$\mathrm{mcx}=$ medial cortex, $\mathrm{lcx}=$ lateral cortex, $\mathrm{cc}=$ corpus callosum, $\mathrm{cf}=$ callosal fibres, $\mathrm{st}=$ striatum, $\mathrm{mth}=$ medial thalamus, $\mathrm{lth}=$ lateral thalamus, $\mathrm{hc}=$ hippocampus, $\mathrm{cb}=$ cerebellum, $\mathrm{bs}=$ brainstem

The estimated longitudinal and transverse relaxation attenuation $\left(f_{\mathrm{T} 1}, f_{\mathrm{T} 2}\right)$ of the brain tissue water, along with the respective LCModel's control parameter atth2o, for exemplary locations, are given in Table 4.3. Relaxation attenuations for water signal were derived by employing measured $T_{1}$ and $T_{2}$ values of water, according to Equations 4.3 and 4.4 .

Table 4.3 Water signal attenuation caused by $T_{1}$ and $T_{2}$ relaxation and pertinent atth 20 estimated for medial cortex and brainstem

\begin{tabular}{llllll}
\hline \hline Structure & $\mathbf{T}_{\mathbf{1}}(\mathbf{m s})$ & $\mathbf{T}_{\mathbf{2}}(\mathbf{m s})$ & $\boldsymbol{f}_{\mathrm{T} 1}$ & $\boldsymbol{f}_{\mathrm{T} 2}$ & atth2o \\
\hline mcx & 2002 & 37 & 0.95 & 0.76 & 0.72 \\
bs & 1709 & 33 & 0.96 & 0.74 & 0.71 \\
\hline \hline
\end{tabular}

mcx $=$ medial cortex, bs $=$ brainstem 
$T_{1}$ values for proton spins of metabolites in aqueous solution are much longer than those in the brain (Michaelis et al., 1993b, Pouwels and Frahm, 1998, Pfeuffer et al., 1999, in 't Zandt et al., 2001). Taken together with the fact that both model spectra and in vivo brain spectra are obtained under fully relaxed conditions, using sufficiently long repetition times, it dispenses the need of correction for differential $T_{1}$ relaxation for absolute metabolite quantification.

With regard to $T_{2}$ relaxation, the total signal loss of water signal at the short TE of $10 \mathrm{~ms}$ is $28 \%$ and $29 \%$ in medial cortex and brainstem, respectively. To account for this attenuation, the corresponding correction factors of 1.03 and 1.01 may be applied in metabolite concentration. Therefore, for all the regions, atth $20=0.7$, i.e., signal loss of $30 \%$, was used, because it is unnecessary to measure $\mathrm{T}_{1}$ relaxation time for each region and animal.

Exemplarily, differential $T_{2}$ attenuations were determined for glutamate and creatine $\mathrm{CH}_{3}$ assuming the corresponding mean $T_{2}$ relaxation times of $190 \mathrm{~ms}$ and 376 $\mathrm{ms}$ in vitro together with $89 \mathrm{~ms}$ and $113 \mathrm{~ms}$ for those in vivo (Xin et al., 2008). The resulting signal loss of $6 \%$ (TE $=10 \mathrm{~ms}$ ) was estimated for these resonances, using Equation 4.6 in Sec. 4.2. Consequently, the related correction factor of only 1.06 may be derived, which required to be multiplied to their concentrations, which was thus neglected in the current work. 


\section{Water content}

The water content of the localized regions was calculated, based on measured $T_{1}$, as suggested earlier by Schwarcz et al in a study of water content quantification of vasogenic edema in mouse brain, at 9.4 Tesla. Equation 4.7 indicates the expected linearity between the inverse of longitudinal relaxation $\left(1 / T_{1}\right)$ of the water protons and the inverse of the total water content $(1 / W)$, where $W$ is the percent concentration of water. This was previously presented and experimentally verified at $400 \mathrm{MHz}$ (Schwarcz et al., 2001).

$$
\frac{1}{T_{1}}=3.106 \frac{1}{W}-3.2998
$$

The molar brain water content, essential for absolute quantification, is derived from the water content (W), assuming a concentration of $55.6 \mathrm{~mol} /$ liter for pure water and brain density of $1.047 \mathrm{~g} / \mathrm{ml}$ (Torack et al., 1976, Brooks et al., 1980, Rieth et al., 1980, Kreis et al., 1993a).

Water longitudinal relaxation times $\left(T_{1}\right)$ in different regions of the mouse brain were determined in vivo at 9.4 $\mathrm{T}$. $\mathrm{T}_{1}$ relaxation times were calculated from selected volumes of interest (VOIs) on a mono exponential fitting basis, according to Equations 4.2 and 4.4. Calculated values ranged from $1709 \mathrm{~ms}$ in brain stem to $2002 \mathrm{~ms}$ in medial cortex. Consequently, corresponding calculated fractional water content, ranged from $80 \%$ to $82 \%$, respectively (Equation 4.7 ). Assuming a pure water concentration of 55.6 mol/liter and brain tissue density of $1.047 \mathrm{~g} / \mathrm{ml}$ (Takagi et al., 1981, Kreis et al., 1993a), water content of 42.5 and $43.5 \mathrm{~mol} /$ liter were yielded for the regions stated before. From the repeated measurements of water content in the cortical area, individual reproducibility error was assessed. The small standard deviations of less than $1 \%$ demonstrated the excellent reproducibility of the experiments. As described in section 4.2, water signal was used as an internal reference for quantification of metabolite concentrations.

The obtained value turned out to be in line with $43.7 \mathrm{~mol} / \mathrm{L}$, determined by Schwarcz et al. (Schwarcz et al., 2001, Schwarcz et al., 2003). Therefore, this value was 
used for the quantification of metabolites from each mouse because the spectroscopic $\mathrm{T}_{1}$ measurement requires considerable prolongation of measuring time. However, the described method will be of essential importance for quantification of metabolites in tissue with altered water concentration, e.g., tumor, edema, or infection.

\section{Reproducibility assessment}

The intra-individual reproducibility study was carried out by acquiring six spectra from the medial cortex region (MCX) of an animal under similar experimental conditions, in three different sessions, spaced several months apart. For the inter-individual study, data was obtained from 9 animals and each, of which, was measured only once. In order to minimize variability, due to voxel positioning, an oblique VOI of $6.75 \mu \mathrm{L}$ was carefully placed in MCX by a single investigator during all the scans. As previously mentioned, a stereotaxic holder, incorporated in a home-built animal slider, was used to appropriately immobilize the head of the anaesthetized mice. The coefficient of variation (CV) (given as $\% C V=(S D / M) \times 100$ where $M$ and SD are the mean and standard deviation of the metabolite concentration reported by LCModel) was determined to characterize intraand inter-individual variability. 


\subsection{Results}

\section{Regional Differences}

High-quality multi-slice RARE images of the mouse brain ensured accurate and reproducible positioning of the $\mathrm{VOI}$ in different brain regions. Representative watersuppressed in vivo proton MR spectra of the investigated mouse brain regions clearly show the well-resolved resonances of numerous cerebral metabolites signals (see Fig. 4.4), obtained with sufficiently consistent spectral quality, e.g., SNR and linewidth, Table 4.5.

The spectra include resonances from Ala, Asp, Cr, PCr, GABA, Glu, Gln, Cho, Ins, Lac, NAA, NAAG and Tau. Considerable spectral variations among the various investigated brain regions can be observed. 

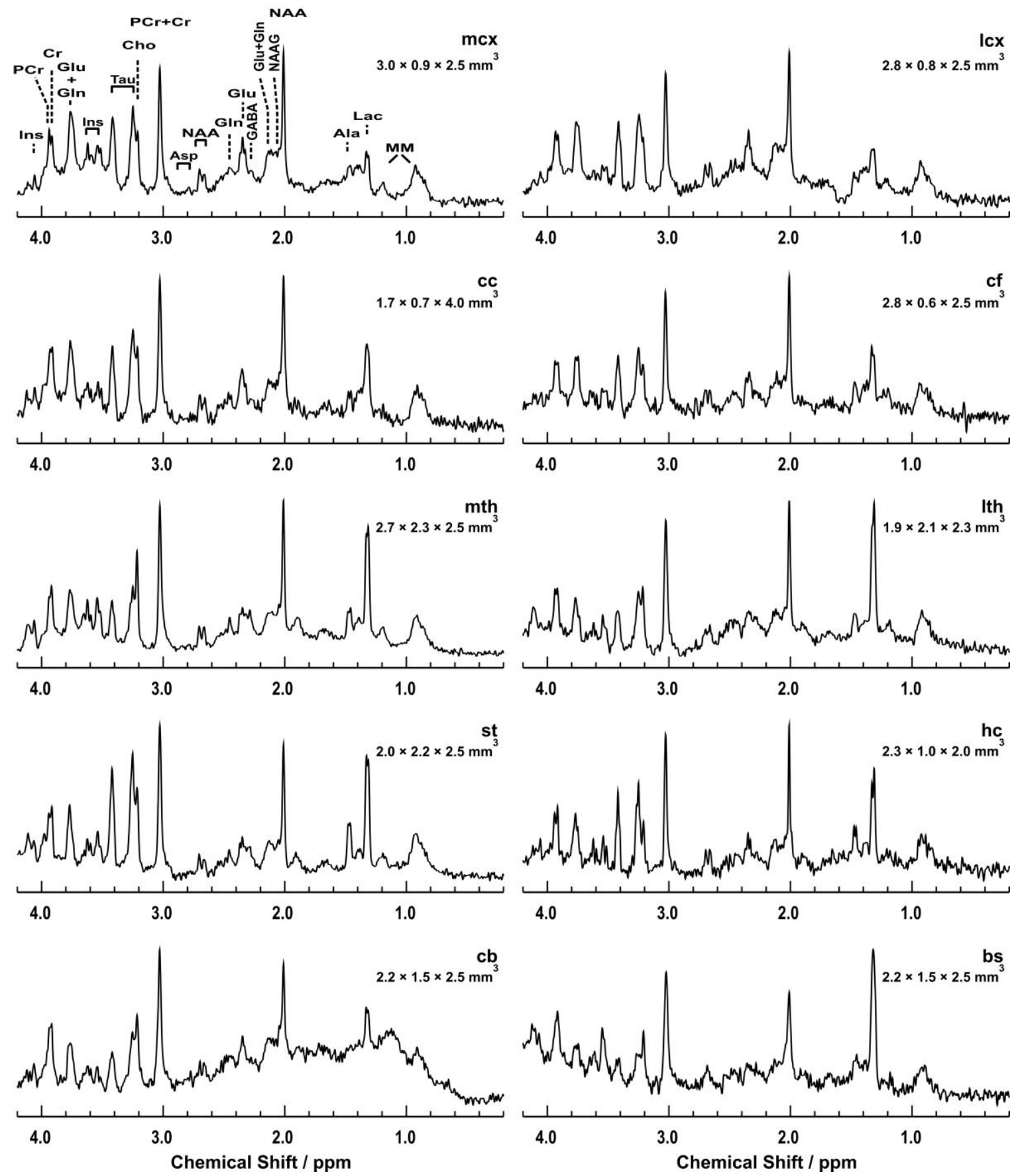

Figure 4.4: Localized proton MR spectra (STEAM, TR/TE/TM=6000/10/10 ms, 4.2 to $15.5 \mu \mathrm{l} \mathrm{VOls,} 2 \times 128$ accumulations per animal) from selected locations in normal mouse brain obtained in vivo at $9.4 \mathrm{~T}$. For illustration purposes only, spectral post-processing comprised of zero-filling to $4 \mathrm{~K}$ data points, mild Lorentz-Gauss apodization in the time-domain (half width $88 \mathrm{msec}$, corresponding to $5 \mathrm{~Hz}$ line broadening) and phase correction according to LCModel. Spectra were scaled in absolute units in proportion to the brain water concentration. 
The LCModel analysis of a typical proton MR spectra from the thalamus of a NMRI mouse brain are presented in Figure 4.5 to illustrate the principle of employed fitting method in the frequency domain. The fitted spectrum is decomposed into its component model spectra from individual brain metabolites, which are individually scaled. The fitting residual, i.e. the difference between the measured and fitted spectra, is small, flat and artifact-free, which is a clear indication of the excellent fit by LCModel, achieved through a proper inclusion of metabolites model spectrum in the basis set. Flat macromolecular "baseline" estimated by LCModel, specifically in the spectral region from 0.5 to $2.0 \mathrm{ppm}$, is an indication of its successful simulation of fast-relaxing signals from macromolecule.

Furthermore, the C4 proton resonances of Glu (2.35 ppm) were completely resolved from those of $\mathrm{G} \ln (2.45 \mathrm{ppm})$. Additionally, the GABA C4 resonance at $2.28 \mathrm{ppm}$ was discernible from the resonance of Glu C4. And its C3 quintet, centered at $1.89 \mathrm{ppm}$, was clearly discriminated from the signal of NAA. The latter was most notably observed in the thalamus and striatum, where GABA concentrations are maximally, and it was least notably detected in other regions where GABA was hardly visible. 
residual

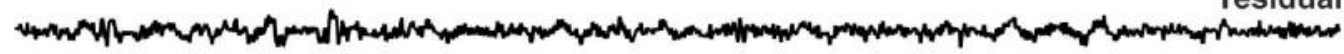
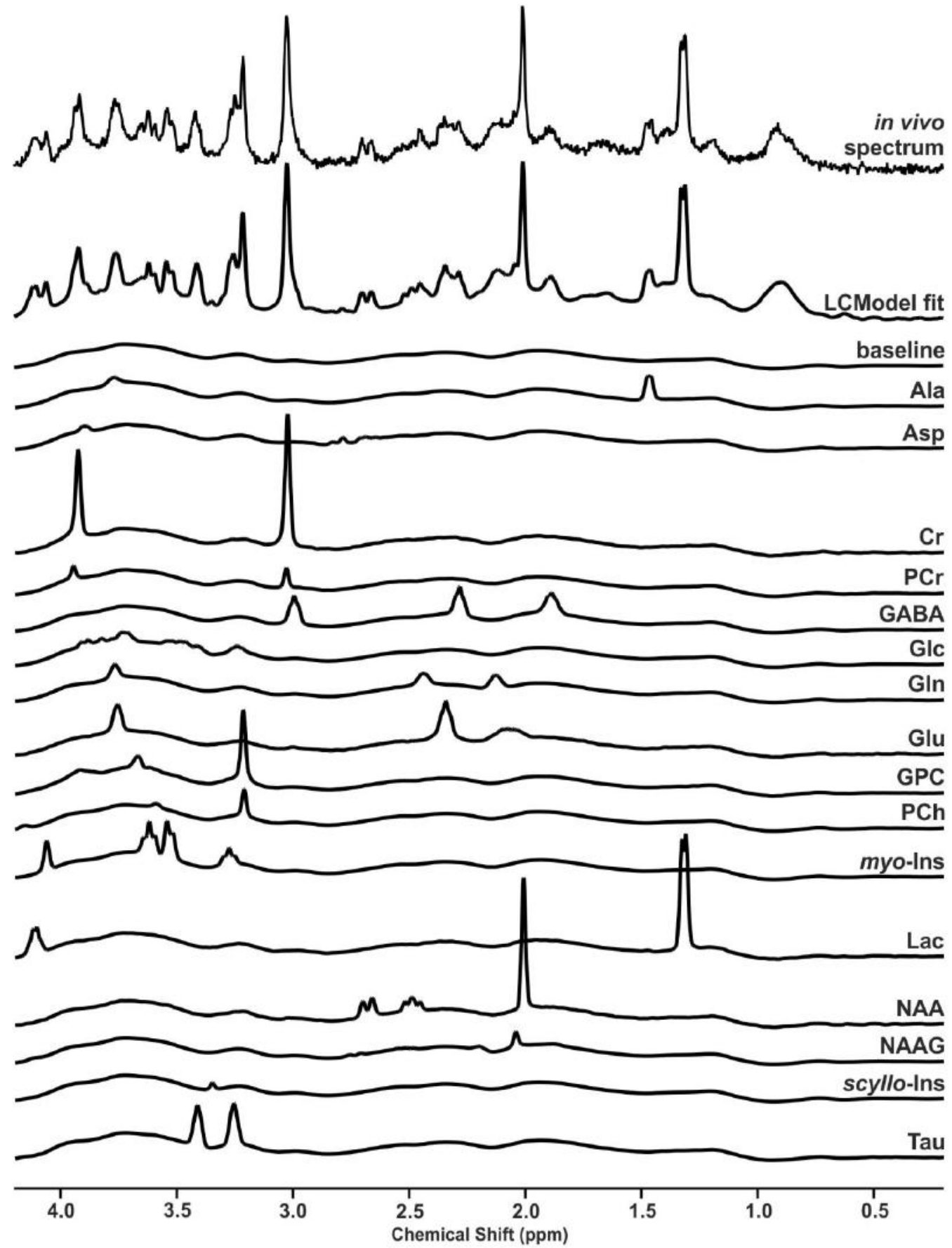

Figure 4.5: LCModel fit of a representative proton MR spectrum from the thalamus of the mouse brain in vivo at 9.4 T obtained with use of a short-echo time STEAM sequence (see Fig. 3.5). From the top: residuals, in vivo spectrum, fitted spectrum, macromolecules, and spectral contributions of individual metabolites to the total spectral fit. All spectra are scaled consistently. See text for abbreviations. 
Basic spectral parameters, determined by LCModel, were compared in Table 4.4 to demonstrate detailed regional differences in the achieved spectral quality so that its impact on reliability of the concentration estimates could be evaluated. Mean metabolite linewidths, determined from full linewidth at half maximum (FWHM) of total creatine singlet at $3.03 \mathrm{ppm}$, reflect the results of localized shimming using FASTMAP.

Signal-to-noise ratios (SNRs) were calculated in frequency domain, using the maximum height of signal at the position of NAA methyl resonance at $2.008 \mathrm{ppm}$, divided by the root mean square (rms) of the residues. Mean linewidths vary across the investigated regions, however this observation was independent of the VOIs size and mainly depended on VOI locations and dimension. First of all, tissue heterogeneities within selected VOIs can cause line broadening. For example, the VOI of $170 \times 700 \times$ $4000 \mu \mathrm{m}^{3}$ was selected for the corpus callosum, which ensures a reasonable covering of the structure, while providing sufficient SNR within a limited measuring time. However, the actual volume of white matter in this VOI is much smaller. This means that different types of tissue (e.g., cell body assemblies, neuropils), other than the white matter, are included in the VOI, which may lead to magnetic field heterogeneities due to susceptibility differences. The placement of VOI within pure white matter is excluded, because the dimension of pure white matter can be estimated to be $250 \times 100 \times 500$ $\mu \mathrm{m}^{3}$ (Michaelis et al., 2009). The selection of such small VOI attenuates the SNR by a factor of 38 and therefore causes unrealistic measuring time. Further, increased FWHM in corpus callosum, callosal fibres and cerebellum can be partly explained by the need for positioning larger cubical volume of FASTMAP which encompassed the other undesired brain region rather than the selected VOI itself. Apart from that, localized shimming is impaired in BS, due to the fact that it is located further away from the surface coil. The resulting lower SNR deteriorates the shimming performance of FASTMAP.

As described in the methods, the mean CRLBs of LCModel analysis across all metabolites were utilized to assess the reliability of metabolite quantification. The mean of the estimates of fitting error expressed in \% are consistent with those expressed in units of concentration ( $\mathrm{mmol} /$ liter VOI). As expected, it is noticed that the precision of 
metabolite quantification slightly degraded [deteriorated] in the location with the lower SNRs and larger linewidths, e.g., in the callosal fibers and brainstem.

Table 4.4. Comparison of metabolite linewidths and SNR of spectra together with CRLB determined using LCModel from different mouse brain region to characterize the achieved spectral quality and the reliability of metabolite quantification.

\begin{tabular}{|c|c|c|c|c|c|c|c|c|c|c|}
\hline & \multicolumn{10}{|c|}{ Structure } \\
\hline & $\mathrm{mcx}$ & lcx & $\mathrm{cc}$ & cf & st & mth & lth & hc & $\mathrm{cb}$ & bs \\
\hline & $\mathrm{n}=8$ & $\mathrm{n}=7$ & $\mathrm{n}=9$ & $\mathrm{n}=8$ & $\mathrm{n}=8$ & $n=9$ & $\mathrm{n}=9$ & $\mathrm{n}=9$ & $\mathrm{n}=8$ & $\mathrm{n}=5$ \\
\hline Volume $(\mu \mathrm{l})$ & 6.8 & 5.6 & 4.8 & 4.2 & 11.0 & 15.5 & 9.2 & 4.6 & 6.1 & 8.3 \\
\hline SNR & 14.1 & 12.4 & 8.6 & 7.7 & 13.9 & 18.4 & 11.7 & 9.4 & 11.9 & 7.8 \\
\hline FWHM $(\mathrm{Hz})$ & 8.8 & 10.4 & 12.4 & 11.6 & 9.6 & 8.0 & 8.4 & 7.2 & 9.6 & 12.0 \\
\hline CRLB (\%) & 9.8 & 10.2 & 12 & 12.7 & 9.9 & 9.3 & 11.3 & 12 & 13.6 & 15.5 \\
\hline CRLB (mM) & 0.29 & 0.3 & 0.4 & 0.43 & 0.3 & 0.27 & 0.34 & 0.4 & 0.45 & 0.51 \\
\hline
\end{tabular}

$\mathrm{mcx}=$ medial cortex, $\mathrm{lcx}=$ lateral cortex, $\mathrm{cc}=$ corpus callosum, $\mathrm{cf}=$ callosal fibres, $\mathrm{st}=$ striatum, $\mathrm{mth}=$ medial thalamus, $\mathrm{lth}=$ lateral thalamus, $\mathrm{hc}=$ hippocampus, $\mathrm{cb}=$ cerebellum, $\mathrm{bs}=$ brainstem

Absolute concentrations (mean \pm SD) of 16 metabolites were reliably quantified in different brain regions, which were obtained by the LCModel analysis of all acquired in vivo ${ }^{1} \mathrm{H}$ NMR spectra (NT $=256$ ) from nine animals measured at $9.4 \mathrm{~T}$. Results of quantitative evaluation of metabolites, along with four combined pairs of metabolites, are illustrated in Figure 4.6.

The corresponding average Cramer-Rao lower bounds (CRLB), which are the approximations of fitting errors and uncertainties of metabolite concentrations, were below $20 \%$ for most of the metabolites, for all regions.

Total NAA, i.e., N-acetylaspartate and N-acetylaspartylglutamate (tNAA, NAA + NAAG), total creatine, i.e., creatine and phosphocreatine ( $\mathrm{tCr}, \mathrm{Cr}+\mathrm{PCr}$ ), total choline, i.e., glycerophosphocholine and phosphocholine (tCho, GPC + PCh), combined glutamate and glutamine $(\mathrm{Glx}, \mathrm{Glu}+\mathrm{Gln})$, taurine (Tau), NAA, Cr, Glu and lactate (Lac) were quantified with average CRLB $<8 \%$.

For some weakly represented metabolites, CRLB was greater than $20 \%$ in some brain regions. The CRLB was between $6 \%$ and $40 \%$ for alanine (Ala), while it was in the range of $15-30 \%$ for NAAG. However, $\gamma$-aminobutyric acid (GABA) concentration was measured with the estimate of fitting error ranging from 5 to $15 \%$. Aspartate and 
glucose were only detected (CRLB $<50 \%$ ) in three and four animals for the striatum, respectively.

In spite of the fact that scyllo-Ins signal was incorporated into the basis set of LCModel as a model component, corresponding concentration was only possible to quantify in the medial thalamus $(0.19 \pm 0.01 \mathrm{mM}, \mathrm{N}=3)$; thus, this metabolite was not further evaluated and it was disregarded from the bar diagram in Figure 4.6. 

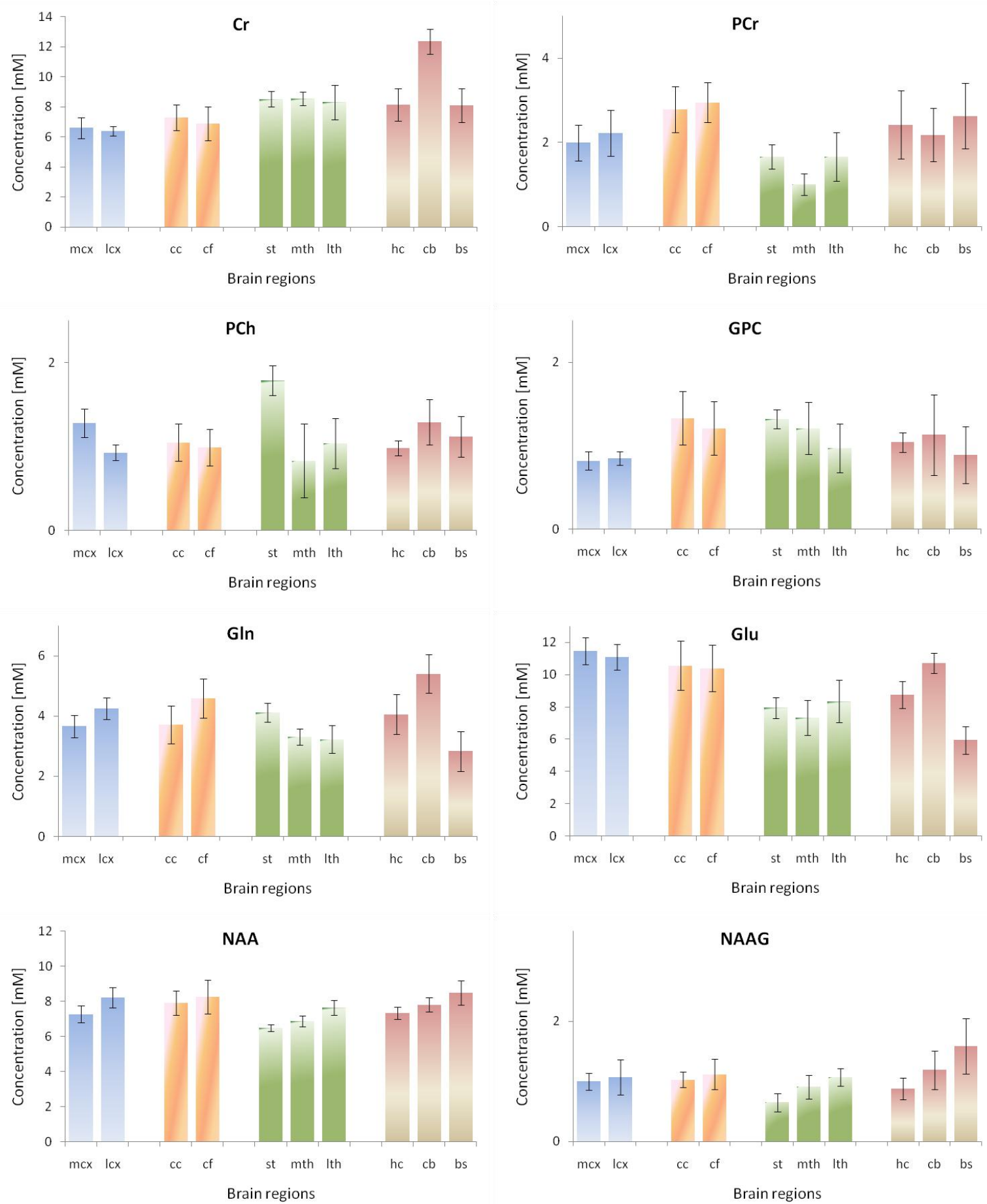

Figure 4.6: Cerebral metabolite concentrations (in $\mathrm{mM}$ per liter VOI) determined by LCModel analysis of the in vivo ${ }^{1} \mathrm{H}$ NMR spectra of ten different regions in brain of mice. Error bars indicate standard deviations. $\mathrm{mcx}=$ medial cortex, $\mathrm{Icx}=$ lateral cortex, $\mathrm{cc}=$ corpus callosum, $\mathrm{cf}=$ callosal fibres, $\mathrm{st}=$ striatum, $\mathrm{mth}=$ medial thalamus, Ith = lateral thalamus, $\mathrm{hc}=$ hippocampus, $\mathrm{cb}=$ cerebellum, $\mathrm{bs}=$ brainstem . 

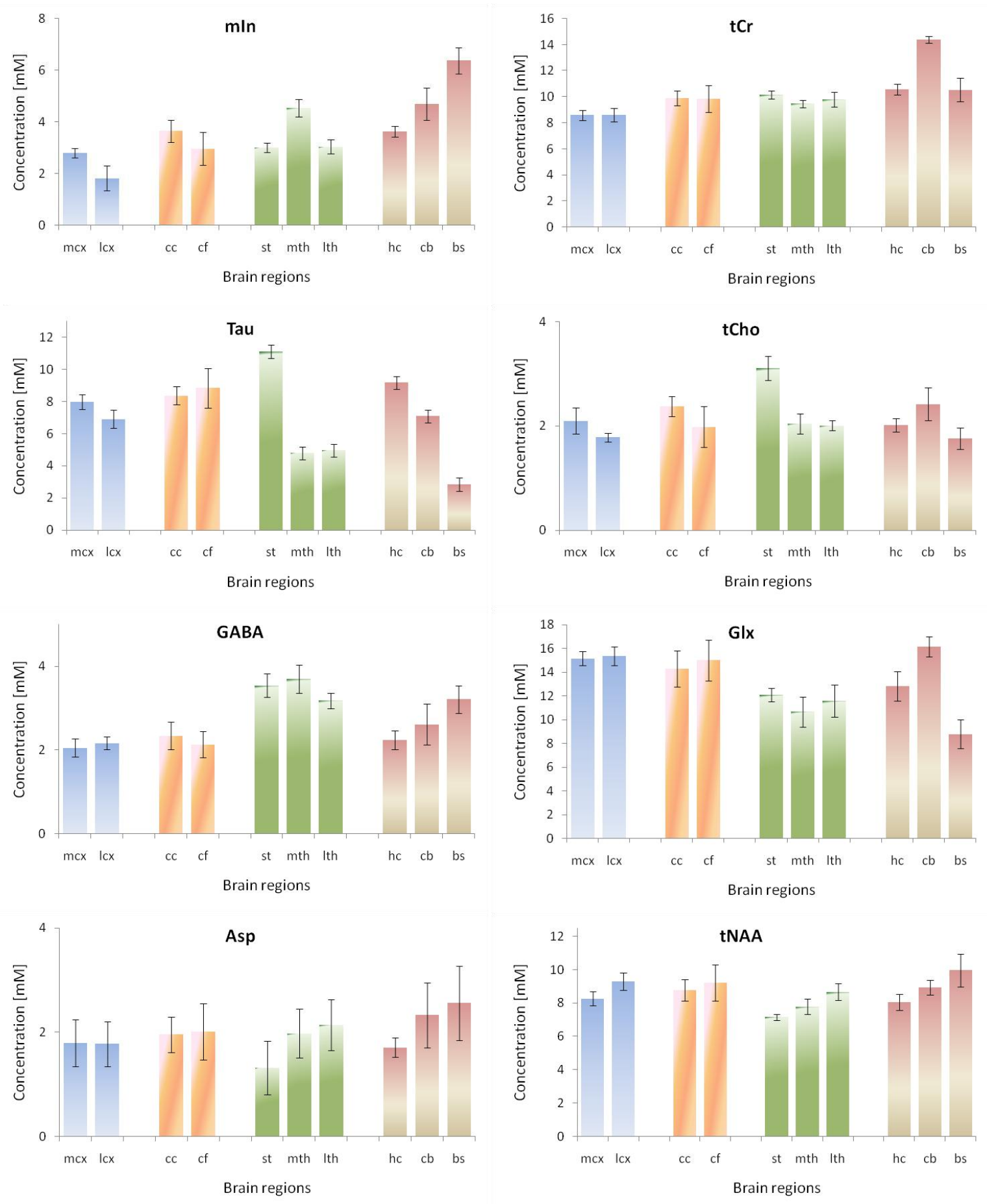

Figure 4.6: Continued. 

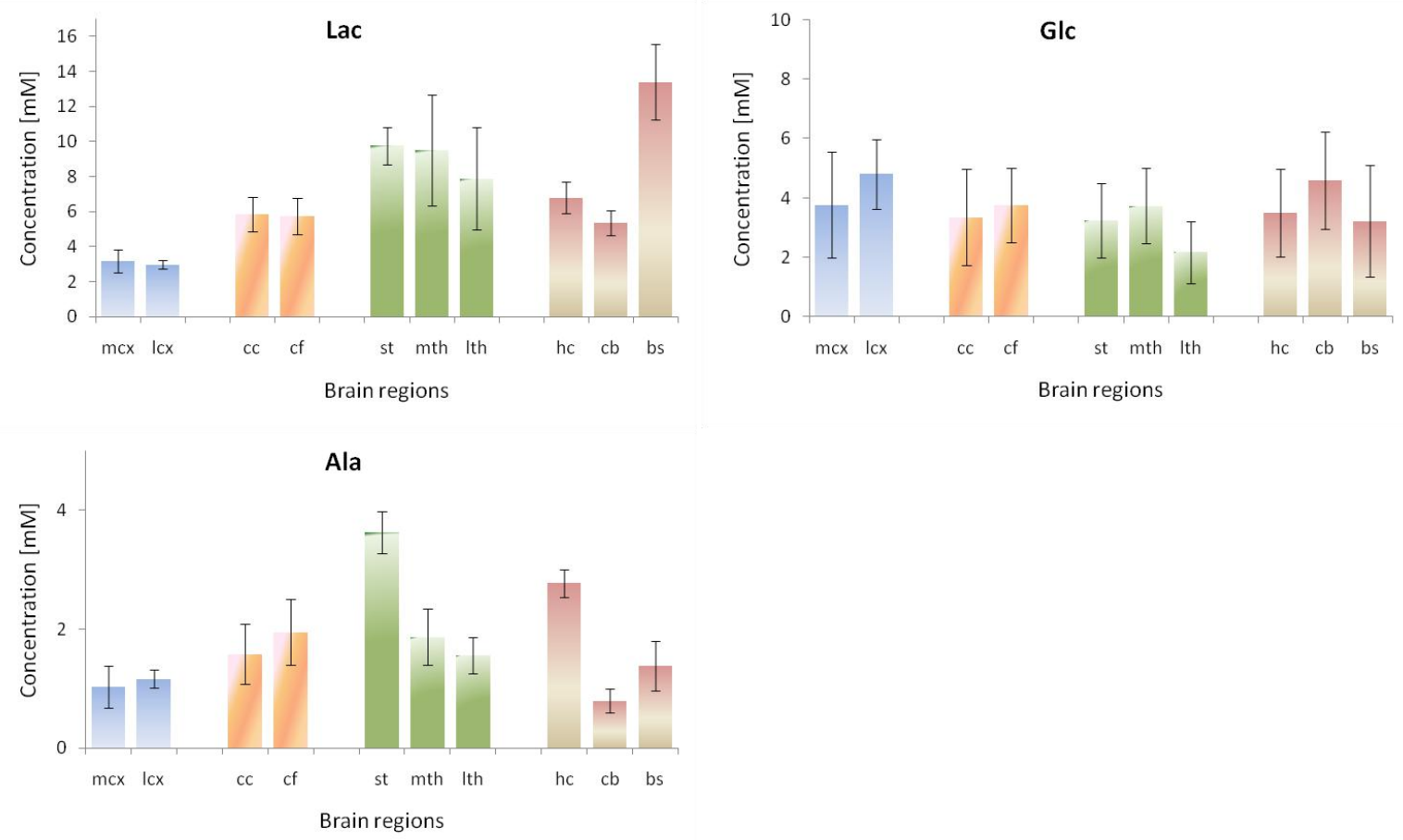

Figure 4.6: Continued.

More detailed information on absolute metabolite concentration estimates, along with corresponding Crame'r-Rao lower bounds of LCModel, are given in Table 4.5. 


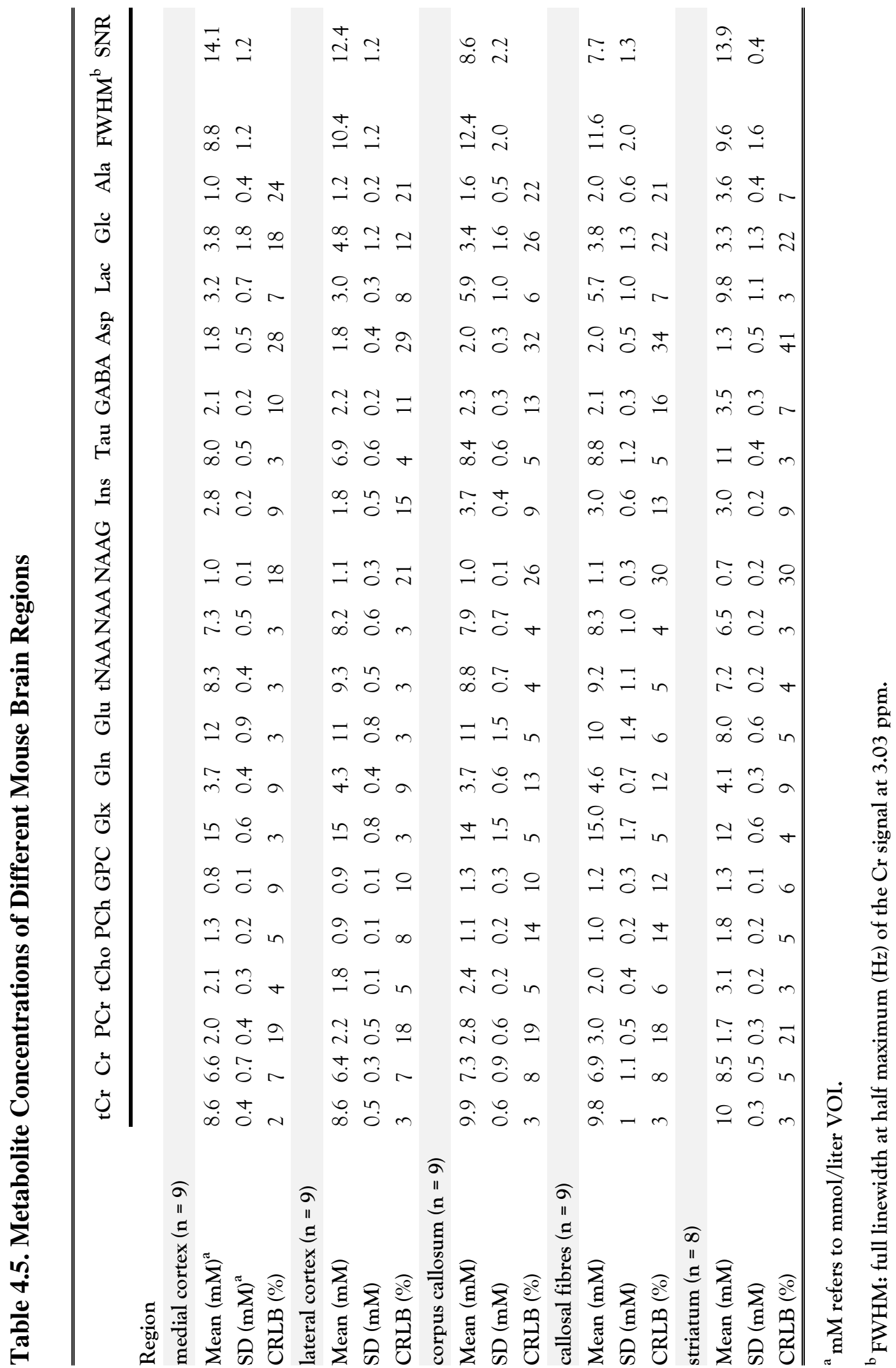




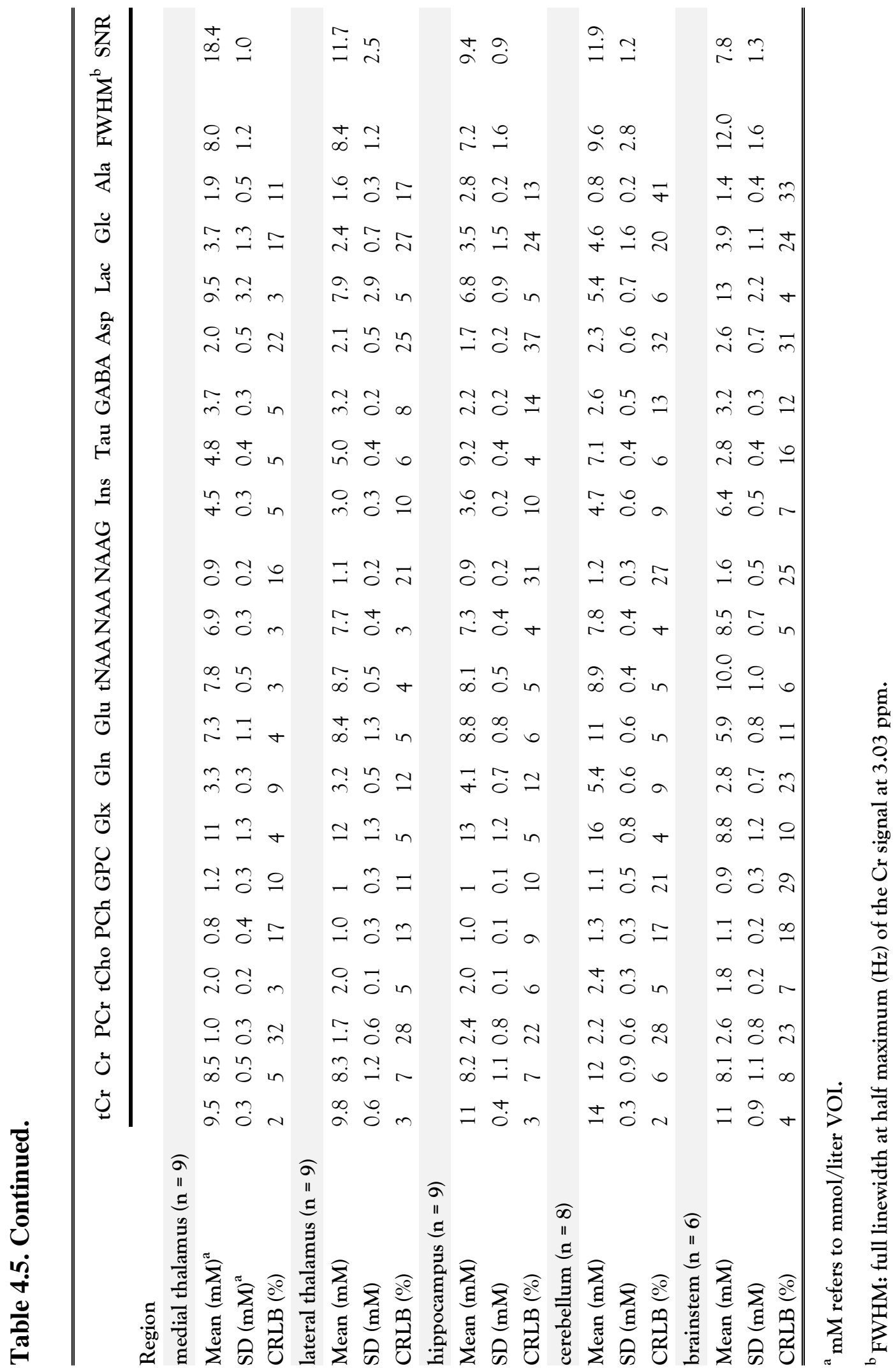




\section{Reproducibility}

Figure 4.7 shows spectra obtained from the same VOI in the medial cortex region of an individual animal on three different occasions. The bottom trace represents the spectra from back-to-back measurements on each session. The spectral quality was highly reproducible, as indicated by the resemblance among all the spectra.
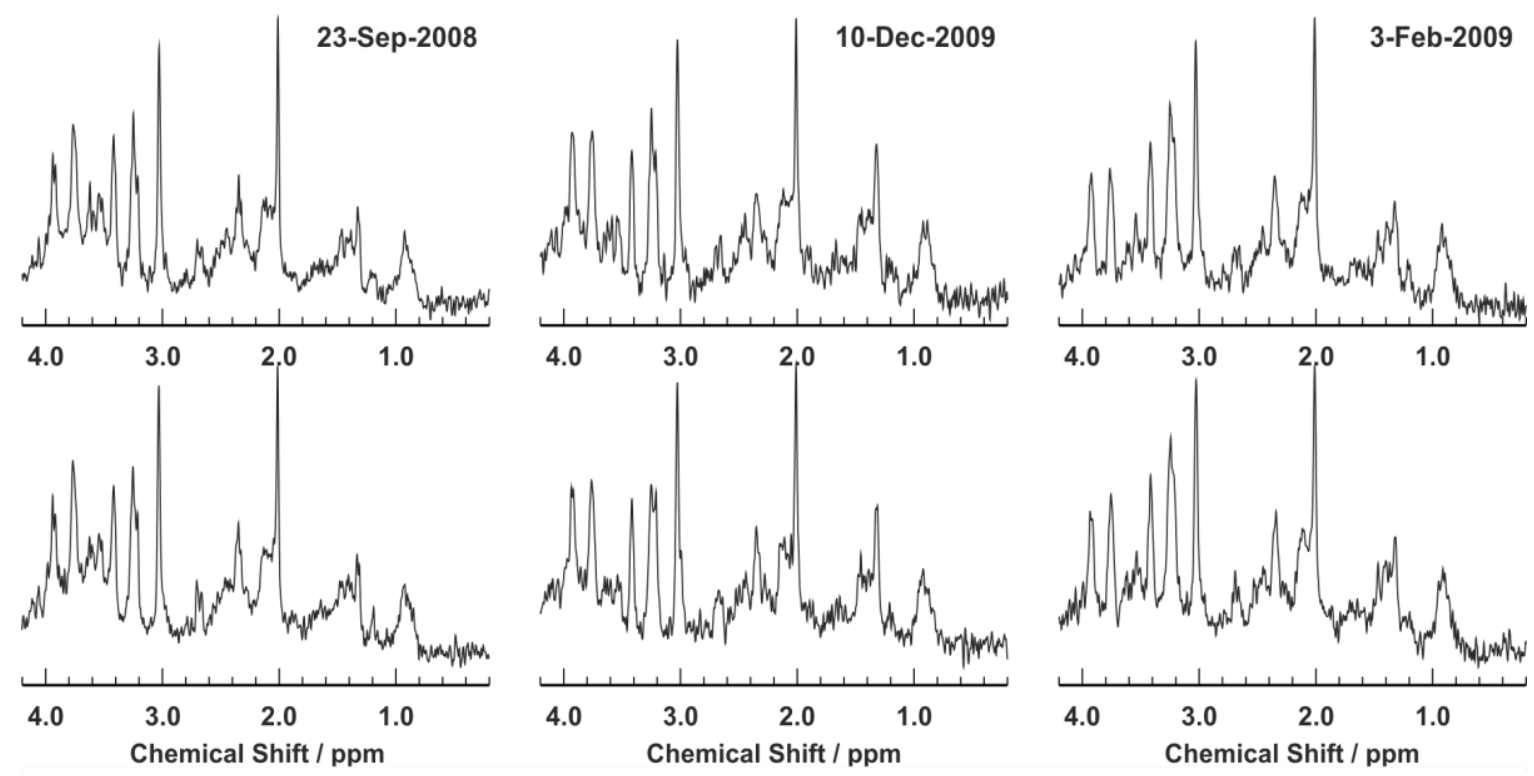

Figure 4.7: Representative in vivo ${ }^{1} \mathrm{H}$ NMR spectra from $6.75 \mu \mathrm{L}$ volume in the medial cortex of an individual animal from three different sessions (top trace) and their corresponding spectra from back-toback measurements (bottom trace). Acquisition and processing parameters as well as peak assignments are the same as in Fig. 4.4.

The intra- and inter-individual variability of metabolite concentrations as well as the respective mean CRLB values are summarized in Table 4.6 for medial cortex. 
Table 4.6 Absolute metabolite concentrations (mean \pm SD), the coefficients of variation (CV) and Crame'r-Rao lower bounds (CRLB) from the intra- and interindividual studies, obtained by LCModel analysis of in vivo NMR spectra from medial cortex region of the healthy mouse brain.

\begin{tabular}{|c|c|c|c|c|c|c|}
\hline \multirow[b]{2}{*}{ Metabolite } & \multicolumn{3}{|c|}{$\operatorname{intra}(n=6)$} & \multicolumn{3}{|c|}{ inter $(n=9)$} \\
\hline & $\begin{array}{l}\text { Mean } \pm \text { SD } \\
(\mathrm{mM})\end{array}$ & $\begin{array}{l}\mathrm{CV} \\
(\%)\end{array}$ & $\begin{array}{l}\text { CRLB } \\
(\%)\end{array}$ & $\begin{array}{l}\text { Mean } \pm \text { SD } \\
(\mathrm{mM})\end{array}$ & $\begin{array}{l}\mathrm{CV} \\
(\%)\end{array}$ & $\begin{array}{l}\text { CRLB } \\
\text { (\%) }\end{array}$ \\
\hline Ala & $1.17 \pm 0.43$ & 37 & 29 & $1.07 \pm 0.39$ & 36 & 29 \\
\hline GABA & $2.24 \pm 0.30$ & 13 & 12 & $2.10 \pm 0.26$ & 12 & 12 \\
\hline Glc & $3.99 \pm 1.22$ & 31 & 18 & $3.72 \pm 1.79$ & 48 & 20 \\
\hline Tau & $7.67 \pm 0.43$ & 6 & 4 & $8.24 \pm 0.68$ & 8 & 4 \\
\hline $\mathrm{NAA}$ & $7.45 \pm 0.44$ & 6 & 4 & $7.46 \pm 0.57$ & 8 & 3 \\
\hline NAAG & $0.94 \pm 0.26$ & 28 & 26 & $0.95 \pm 0.22$ & 23 & 24 \\
\hline $\mathrm{Cr}$ & $6.50 \pm 0.70$ & 11 & 7 & $6.25 \pm 1.16$ & 19 & 8 \\
\hline $\mathrm{PCr}$ & $2.11 \pm 0.49$ & 23 & 22 & $2.37 \pm 0.70$ & 30 & 19 \\
\hline Gln & $4.40 \pm 0.32$ & 7 & 9 & $3.60 \pm 0.35$ & 10 & 11 \\
\hline Glu & $10.93 \pm 0.63$ & 6 & 4 & $11.61 \pm 0.96$ & 8 & 4 \\
\hline GPC & $0.95 \pm 0.10$ & 11 & 10 & $0.83 \pm 0.13$ & 16 & 11 \\
\hline PCh & $1.19 \pm 0.11$ & 9 & 8 & $1.26 \pm 0.19$ & 15 & 7 \\
\hline Ins & $2.78 \pm 0.43$ & 15 & 11 & $2.79 \pm 0.21$ & 8 & 10 \\
\hline Lac & $3.27 \pm 0.92$ & 28 & 9 & $3.13 \pm 0.72$ & 23 & 9 \\
\hline Asp & $1.92 \pm 0.31$ & 16 & 29 & $2.00 \pm 0.59$ & 30 & 29 \\
\hline $\mathrm{NAA}+\mathrm{NAAG}$ & $8.38 \pm 0.40$ & 5 & 4 & $8.41 \pm 0.61$ & 7 & 4 \\
\hline $\mathrm{Cr}+\mathrm{PCr}$ & $8.60 \pm 0.35$ & 4 & 3 & $8.62 \pm 0.55$ & 6 & 3 \\
\hline $\mathrm{GPC}+\mathrm{PCh}$ & $2.14 \pm 0.20$ & 9 & 5 & $2.09 \pm 0.29$ & 14 & 5 \\
\hline Glu+Gln & $15.33 \pm 0.54$ & 4 & 4 & $15.21 \pm 0.90$ & 6 & 4 \\
\hline
\end{tabular}

The intra-individual coefficient of variation (CV) for most metabolites (NAA, $\mathrm{Cr}$, Glu, Gln, Tau, GPC, PCh, tCr, tCho, tNAA, Glu+Gln) was $\leq 11 \%$ and was below $15 \%$ for GABA and myo-Ins. Additionally, this value ranged from $16 \%$ to $37 \%$ for weakly represented metabolites (Asp, NAAG, Ala). These are in agreement with results of the rat brain, reported by Pfeuffer and Hong at field strengths of $9.4 \mathrm{~T}$ and $16.4 \mathrm{~T}$, respectively (Pfeuffer et al., 1999, Hong et al., 2011b). The inter-individual CV was found to be rather similar to that observed in the study of intra-individual variability.

The lower CV of the summed metabolites indicates that the resonances of the related individual components are obscured by spectral overlap and hence, were only partially resolved. This is further characterized by decreased mean CRLB (estimate of the 
fitting error) for the summed metabolites, compared to their individual values in both intra- and inter-individual reproducibility studies, as can be seen in Table 4.2.

The CRLBs estimated by LCModel were consistent with the CVs values, measured for each metabolite concentration. The mean CRLB values for all metabolites were nearly identical in intra- and inter-individual studies, representing the reliability and robustness of the LCModel fitting analysis, from a single spectra, to quantify the metabolite concentrations within and across the same animals. 


\subsection{Discussion}

Care was taken to optimize dimension of VOls for every region to ensure better localization and to include maximum area of the brain structure. This leads to diminished partial volume effects and avoids ventricular space contribution, within the selected volume. However, the remnant uncompensated susceptibility results in inhomogeneous line broadening and in deterioration of the achievable spectral linewidths, which are markedly noticeable in locations with strong susceptibility gradients. This may be caused by air-tissue interfaces in inferior regions of the brain or it may be induced by paramagnetic properties of blood, close to the vessels. This influence is manifested as a distortion in magnetic field homogeneity which thus, limits spectral resolution and increases resonance linewidths from $7.2 \pm 1.6 \mathrm{~Hz}$ for $\mathrm{tCr}$ obtained in hippocampus to $12.0 \pm 1.6 \mathrm{~Hz}$ and $12.4 \pm 1.6 \mathrm{~Hz}$ attained in brainstem and corpus callosum, respectively (see Table 4.4). As far as the optimization of the hardware is concerned, the replacement of the standard $1 \mathrm{~kW}$ RF power amplifier by that of $2 \mathrm{~kW}$ allowed shortening of the slice-selection RF pulse duration, which greatly reduced the extent of the chemical shift displacement error to below $10 \%$ of the voxel dimension.

The high spectral quality achieved over the entire chemical shift range (0.5-4.2 ppm) ensured reliable and reproducible quantification of each of the brain metabolites. Signals of many metabolites were clearly resolved as a consequence of increased chemical shift dispersion at 9.4 T. For example, this is reflected in the complete separation of the coupled resonances of Tau from myo-inositol resonances at $3.42 \mathrm{ppm}$. Furthermore, the $\mathrm{C} 4$ proton resonances of Glu $(2.35 \mathrm{ppm})$ were completely resolved from those of Gln (2.45 ppm). Additionally, the GABA C4 resonance at $2.28 \mathrm{ppm}$ was discernible from the resonance of Glu C4. And its C3 quintet centered at $1.89 \mathrm{ppm}$ was clearly discriminated from the signal of NAA, notably in the thalamus and striatum where the maximum GABA concentration observed and hardly visible in other structures.

Furthermore, the singlet resonances from methylene-protons of creatine and phosphocreatine, differing by $0.02 \mathrm{ppm}$, were partially resolved at $3.9 \mathrm{ppm}$ (Gruetter et al., 1998, Pfeuffer et al., 1999, Tkáć et al., 2003) and they were discernible in the ${ }^{1} \mathrm{H}$ NMR spectra, obtained from most of the brain regions. Here, it may be worth noting that the 
strong negative correlation between $\mathrm{Cr}$ and $\mathrm{PCr}$, as estimated from the covariance matrix by the LCModel, would imply a decrease in reliability of individual quantification. In fact, this can give rise to overestimation of one constituent concentration, to the detriment of underestimation of the other (Hofmann et al., 2002, Tkáć et al., 2009). However, the sum of $\mathrm{Cr}$ and $\mathrm{PCr}$ concentration were quantified with high precision. The measured concentrations of $\mathrm{tCr}$ in the current study are in good agreement with previously published concentration values for normal mouse (Renema et al., 2003, Schwarcz et al., 2003, Öz et al., 2010) and rat brain in vivo (Pfeuffer et al., 1999, Tkáć et al., 1999, Hong et al., 2011a, Hong et al., 2011b, Hong et al., 2011c). Similar arguments hold true for signal from tCho at $3.2 \mathrm{ppm}$, which comprises resonances predominantly from the trimethyl amine $\mathrm{N}-\left(\mathrm{CH}_{3}\right)_{3}$ groups of $\mathrm{GPC}$ and $\mathrm{PC}$, although an even stronger LCModel correlation coefficient of $<-0.85$ may lead to large uncertainties in measurement of the individual constituent.

In addition to notable reductions in scan time, increased sensitivity and spectral resolution at $9.4 \mathrm{~T}$, compared to those at $2.35 \mathrm{~T}$, resulted in a substantial improvement in accuracy and precision of the quantification of metabolites - particularly for those weakly represented. The higher CRLB observed for metabolites with J-coupled spin systems, compared to uncoupled ones, can be explained by the pronounced splitting of their resonances, which considerably overlap with those of more abundant metabolites. A plausible explanation emerges from the analytical expressions of the CRLBs on spectral parameters, as derived by Cavassila, demonstrating the influence of overlap on the model parameter estimates (Cavassila et al., 2000, Cavassila et al., 2001, Cavassila et al., 2002, Kreis, 2004). In this context, it is important to note that the larger standard deviations in metabolite concentration values, obtained in e.g., the brainstem and corpus callosum, can be attributed to systematic errors introduced by reduced SNR and increased line width (see Table 4.4 and Fig. 4.6). Indeed, overestimation of weakly represented metabolites as a function of SNR has been reported by Tkáć et al (Tkáć et al., 2002). Influences of linewidth and SNR on estimated metabolites concentration have also been shown (Kreis and Boesch, 2003, Bartha, 2007). 
The observed regional differences of metabolite concentrations in vivo are in line with literature data, except for tCho (choline-containing compounds, i.e., GPC + PC), Lac, and Ala. The observation of elevated tCho concentrations in the striatum, reported here for the first time, was highly reproducible and consistent among animals, while the concentration of about $2.0 \mathrm{mM}$ in the hippocampus, thalamus, and medial cortex is higher than expected from a previous report (Tkáć et al., 2004). These discrepancies may be explained by the difference in the strain of mice used (NMRI in the present report vs. $\mathrm{C} 57 \mathrm{BL} / 6, \mathrm{CBA}$, and $\mathrm{CBA} / \mathrm{BL6}$ in the previous report).

The observation of the pronounced differences in Lac and Ala content, among various brain regions, generally agree with those reported by others in rats (Tkáć et al., 2003) and mice (Tkáć et al., 2004, Boretius et al., 2011) at 9.4 T. A new finding of the present study, however, was markedly higher (37\%) Lac concentration of brainstem, compared to striatum (see Fig. 4.6). These novel findings may be related to the mouse strain used and may also depend on the procedures used for anesthesia. Indeed, the experimental setup which has been established within the frame of this thesis recently provided data, which partially explains the altered cerebral metabolism, under the applied anesthesia (Boretius et al., 2013). The observed alterations can be explained by the effect of used volatile anesthetic, i.e., isoflurane, which may induce a stimulation of adrenergic pathways, in conjunction with an inhibition of the respiratory chain. The higher Lac concentration in the brainstem suggests that the brainstem may be more vulnerable to the induced physiological conditions, possibly because of its high content of adrenergic neurons.

Scyllo-Ins, a potential marker for human cerebral pathology, has been supposed to have a constant concentration, relative to myo-ins. In The concentration of scyllo-Ins was reported to be 0.4-0.6 mM in human brain (Seaquist and Gruetter, 1998), while that of myo-ins is approximated to be about $6 \mathrm{mM}$ (Michaelis et al., 1993a). In the present study, the concentration of scyllo-Ins cannot be quantified with sufficient reliability, except from the medial thalamus $(0.19 \pm 0.01 \mathrm{mM})$ and the detected concentration of myo-ins, ranging between 1.82 and $6.37 \mathrm{mM}$, is in line with previously reported data of both humans and mice (Tkáć et al., 2004). This lack of reliable detection in most regions 
of the brain, presumably due to its low concentration, can be explained by the assumption of Seaquist and Gruetter that scyllo-Ins metabolism may be regulated independently from myo-ins.

The high reproducibility and reliability of the presented quantitative, single-voxel proton MRS measurements in the mice brain at $9.4 \mathrm{~T}$, as emphasized by the small CV and CRLB in Table 4.6, correspond well with previous data (Öz et al., 2010) and hold great promise for the ability to reliably detect subtle changes in metabolite concentration - for example, those associated with the progression of neurodegenerative diseases or therapeutic response to pharmacotherapy. The excellent agreement observed between the CRLB values returned by LCModel and the calculated CV values (see Table 4.6), which were measured for each metabolite concentration, confirms the good quality of the obtained data and the subsequent successful approximation by the model functions.

The lower value for CRLB was considered to stem from the fact that it represents the lower bound on the variance of unbiased estimate of the model parameter in the presence of normally distributed noise and reflects only the statistical uncertainty of the estimate; therefore, the larger scatter (e.g., standard deviation) in the observed data (Table. 4.5 and 4.6) can be explained by the systematic errors, e.g., incorrect prior knowledge and numerous artifacts (Provencher, 1993, Kreis, 1997, Cavassila et al., 2000, Cavassila et al., 2001, Kreis, 2004, Helms, 2008). These are generally not reflected in CRLB. 


\section{Chapter 5}

\section{Summary and Outlook}

The first achievement of this work was implementation and optimization of MRS technique. To achieve this goal, (i) MR spectra, acquired with different bandwidths and inter-pulse delays of water suppression pulses, were systematically investigated for in vitro condition as well as for mouse brain in vivo. Sufficient water suppression allowed a reliable detection of critical metabolite signals, despite their close proximity to the water resonance. Minimum spoiling capacity chosen ensured a sufficient dispersion of transverse water coherences. (ii) The implemented outer-volume-suppression scheme allowed substantial improvement in quantification of metabolites in mouse brain. (iii) The improvement in local static field homogeneity, achieved by FASTMAP shimming, resulted in high and reproducible spectral resolution. (iv) The relative detectability of strongly coupled metabolite resonances was systematically compared between low and high field strengths, using a single phantom and mice of matching strain, gender, and age.

The second achievement was the acquisition of MR spectra and representation of the neurochemical profiles from ten different brain regions of anesthetized mice at 9.4 T. Experimental setup was developed, including the selection of the coils, the method of anesthesia, the maintenance of body temperature, and the fixation of the 
head of mice for repeated MRS from the same mouse and for examining the reproducibility of MRS. VOI localization technique was optimized for different brain regions of anesthetized mice. Measurement of $T_{1}$ and $T_{2}$ relaxation times from different brain regions of anesthetized mice may be used for a correct quantification of metabolite concentrations in order to calculate the partial volume effect of the cerebrospinal fluid, the signal attenuation difference between the tissue in vivo and the metabolite model solution in vitro, and the water content of the tissue in vivo. Absolute concentrations of 15 different brain metabolites from anesthetized mice were presented with their necessary statistical values. Elevated tCho and Ala concentrations in the striatum as well as elevated Lac concentration in the brainstem, so far not reported, were firstly demonstrated. Scyllo-Ins signal was quantified, for the first time, from the brain of mice in vivo. High reproducibility of MRS was demonstrated from the same mouse over four months.

A further reduction in chemical shift displacement error remains an issue, although it was reduced to below $10 \%$ of the voxel dimension by the application of optimal RF power amplifier, together with the shortening of the slice-selective RF pulse, in the present work. An optimal use of adiabatic slice selective RF pulses, e.g., hyperbolic secant pulses, may provide even larger bandwidth, which thus minimizes the chemical shift displacement error.

Further improvement in spectral resolution may be achieved by a phase coherent averaging. Development of an interleaved navigator scan may counteract motion artifacts and magnetic field instabilities. Consequently, SNR improvement is also expected. The signal-to-noise gain may be further expected, when full advantage is taken of phased-array coil (Natt et al., 2005) or of cryo-probe. Further, the basic knowledge acquired here, from comparison between 2.35 T and 9.4 T, will be useful for optimization of MRS at even higher fields.

For the quantification of metabolites, an assumed value of water content was used for all regions because the spectroscopic $T_{1}$ measurement requires considerable measuring time. A faster imaging technique for spin density mapping may be developed to derive water content of each region. This will be of great importance when characterization of pathology with altered water content is required. Furthermore, 
metabolite-nulled spectra can be acquired in vivo (Pfeuffer et al., 1999, Auer et al., 2001, Seeger et al., 2003) and included into the basis set of LCModel, signals from macromolecules can be quantified.

In summary, High-field localized proton NMR spectroscopy, accompanied by LCModel analysis, enabled detection of regional differences in the neurochemical profiles of the normal mouse brain in vivo. This allows detailed studies of metabolic heterogeneity to be conducted in a region-specific manner that hitherto could only have been made on larger animals such as rats and nonhuman primates. The data yield unique non-invasive insights into the intracellular metabolism and the cellular composition of the tissue. The comprehensive data of absolute metabolite concentration presented in this thesis will serve as a reference for all future MRS studies, using behaving mice in a variety of circumstances. Pertinent studies may lead to a better understanding of the pathophysiological mechanisms underlying human neurological and psychiatric disorders. 


\section{Bibliography}

ALGER, J. R., SYMKO, S. C., BIZZI, A., POSSE, S., DESPRES, D. J. \& ARMSTRONG, M. R. (1993) Absolute Quantitation of Short TE Brain ${ }^{1} \mathrm{H}-\mathrm{MR}$ Spectra and Spectroscopic Imaging Data. Journal of Computer Assisted Tomography, 17, 191-199.

AUER, D. P., GÖSSL, C., SCHIRMER, T. \& CZISCH, M. (2001) Improved analysis of ${ }^{1} \mathrm{H}-\mathrm{MR}$ spectra in the presence of mobile lipids. Magnetic Resonance in Medicine, 46, 615-618.

BARKER, P. B., SOHER, B. J., BLACKBAND, S. J., CHATHAM, J. C., MATHEWS, V. P. \& BRYAN, R. N. (1993) Quantitation of proton NMR spectra of the human brain using tissue water as an internal concentration reference. Nmr in Biomedicine, 6, 89-94.

BARTHA, R. (2007) Effect of signal-to-noise ratio and spectral linewidth on metabolite quantification at $4 \mathrm{~T}$. Nmr in Biomedicine, 20, 512-521.

BARTHA, R., DROST, D. J., MENON, R. S. \& WILLIAMSON, P. C. (2000) Comparison of the quantification precision of human short echo time ${ }^{1} \mathrm{H}$ spectroscopy at 1.5 and 4.0 Tesla. Magnetic Resonance in Medicine, 44, 185-192.

BECKER, E. D., FERRETTI, J. A. \& FARRAR, T. C. (1969) Driven equilibrium Fourier transform spectroscopy. A new method for nuclear magnetic resonance signal enhancement. Journal of the American Chemical Society, 91, 7784-\&.

BEHAR, K. L. \& OGINO, T. (1993) Characterization of macromolecule resonances in the ${ }^{1} \mathrm{H}$ NMR spectrum of rat brain. Magnetic Resonance in Medicine, 30, 38-44.

BERKELBACH VAN DER SPRENKEL, J. W., KNUFMAN, N. M., VAN RIJEN, P. C., LUYTEN, P. R., DEN HOLLANDER, J. A. \& TULLEKEN, C. A. (1992) Proton spectroscopic imaging in cerebral ischaemia. Where we stand and what can be expected. Advances and technical standards in neurosurgery, 19, 3-17.

BLOCH, F. (1946) Nuclear Induction. Physical Review, 70, 460-474.

BLOCH, F., HANSEN, W. W. \& PACKARD, M. (1946a) Nuclear Induction. Physical Review, 69, 127127.

BLOCH, F., HANSEN, W. W. \& PACKARD, M. (1946b) The Nuclear Induction Experiment. Physical Review, 70, 474-485. 
BORETIUS, S., TAMMER, R., MICHAELIS, T., BROCKMÖLLER, J. \& FRAHM, J. (2013) Halogenated volatile anesthetics alter brain metabolism as revealed by proton magnetic resonance spectroscopy of mice in vivo. Neuroimage, 69, 244-255.

BORETIUS, S., TAMMER, R., MICHAELIS, T. \& FRAHM, J. (2011) Isoflurane Elevates Brain Lactate in a Dose-dependent Manner: A Localized ${ }^{1} \mathrm{H}$ MRS Study of Mouse Brain In Vivo. 19th Meeting of ISMRM. Montréal.

BOTTOMLEY, P. A. (1987) Spatial localization in NMR spectroscopy in vivo. Ann N Y Acad Sci, 508, 333-48.

BROOKS, R. A., DI CHIRO, G. \& KELLER, M. R. (1980) Explanation of cerebral white--gray contrast in computed tomography. Journal of computer assisted tomography, 4, 489-91.

BROOKS, W. M., FRIEDMAN, S. D. \& STIDLEY, C. A. (1999) Reproducibility of ${ }^{1} \mathrm{H}$-MRS in vivo. Magnetic Resonance in Medicine, 41, 193-197.

C.W. BROOKS, J., ROBERTS, N., KEMP, G. J., MARTIN, P. A. \& WHITEHOUSE, G. H. (1999) Magnetic resonance imaging-based compartmentation and its application to measuring metabolite concentrations in the frontal lobe. Magnetic Resonance in Medicine, 41, 883888.

CAVASSILA, S., DEVAL, S., HUEGEN, C., VAN ORMONDT, D. \& GRAVERON-DEMILLY, D. (2000) Cramér-Rao Bound Expressions for Parametric Estimation of Overlapping Peaks: Influence of Prior Knowledge. Journal of Magnetic Resonance, 143, 311-320.

CAVASSILA, S., DEVAL, S., HUEGEN, C., VAN ORMONDT, D. \& GRAVERON-DEMILLY, D. (2001) Cramér-Rao bounds: an evaluation tool for quantitation. Nmr in Biomedicine, 14, 278283.

CAVASSILA, S., VAN ORMONDT, D. \& GRAVERON-DEMILLY, D. (2002) Cramér-Rao-Bound Analysis of Spectroscopic Signal Processing Methods. Signal Processing for Magnetic Resonance Imaging and Spectroscopy. CRC Press.

CERDÁN, S., PARRILLA, R., SANTORO, J. \& RICO, M. (1985) ${ }^{1} \mathrm{H}$ NMR detection of cerebral myoinositol. FEBS Letters, 187, 167-172.

CHOI, C. G. \& FRAHM, J. (1999) Localized proton MRS of the human hippocampus: Metabolite concentrations and relaxation times. Magnetic Resonance in Medicine, 41, 204-207.

CONNELLY, A., COUNSELL, C., LOHMAN, J. A. B. \& ORDIDGE, R. J. (1988) Outer volume suppressed image related in vivo spectroscopy (OSIRIS), a high-sensitivity localization technique. Journal of Magnetic Resonance (1969), 78, 519-525.

CZEH, B., MICHAELIS, T., WATANABE, T., FRAHM, J., DE BIURRUN, G., VAN KAMPEN, M., BARTOLOMUCCI, A. \& FUCHS, E. (2001) Stress-induced changes in cerebral metabolites, hippocampal volume, and cell proliferation are prevented by antidepressant treatment with tianeptine. Proceedings of the National Academy of Sciences of the United States of America, 98, 12796-12801.

CZEH, B., PUDOVKINA, O., VAN DER HART, M. G. C., SIMON, M., HEILBRONNER, U., MICHAELIS, T., WATANABE, T., FRAHM, J. \& FUCHS, E. (2005) Examining SLV-323, a novel NK1 receptor antagonist, in a chronic psychosocial stress model for depression. Psychopharmacology, 180, 548-557.

DAMADIAN, R. (1971) Tumor Detection by Nuclear Magnetic Resonance. Science, 171, 11511153.

DANIELSEN, E. R. \& HENRIKSEN, O. (1994) Absolute quantitative proton NMR spectroscopy based on the amplitude of the local water suppression pulse. Quantification of brain water and metabolites. Nmr in Biomedicine, 7, 311-318.

DE GRAAF, R. A. (2007) In Vivo NMR Spectroscopy: Principles and Techniques, Wiley-Interscience.

DE GRAAF, R. A., BRAUN, K. P. J. \& NICOLAY, K. (2001) Single-shot diffusion trace ${ }^{1} \mathrm{H}$ NMR spectroscopy. Magnetic Resonance in Medicine, 45, 741-748. 
DI COSTANZO, A., TROJSI, F., TOSETTI, M., GIANNATEMPO, G. M., NEMORE, F., PICCIRILLO, M., BONAVITA, S., TEDESCHI, G. \& SCARABINO, T. (2003) High-field proton MRS of human brain. European journal of radiology, 48, 146-153.

DICKINSON, W. C. (1950) Dependence of the $\mathrm{F}^{19}$ Nuclear Resonance Position on Chemical Compound. Physical Review, 77, 736-737.

DUIJN, J., MATSON, G., MAUDSLEY, A., HUGG, J. \& WEINER, M. (1992) Human brain infarction: proton MR spectroscopy. Radiology, 183, 711.

DUYN, J., GILLEN, J., SOBERING, G., VAN ZIJL, P. \& MOONEN, C. (1993) Multisection proton MR spectroscopic imaging of the brain. Radiology, 188, 277.

ERNST, T. \& HENNIG, J. (1995) Improved water suppression for localized in vivo ${ }^{1} \mathrm{H}$ spectroscopy. Journal of Magnetic Resonance Series B, 106, 181-186.

ERNST, T., KREIS, R. \& ROSS, B. D. (1993) Absolute Quantitation of Water and Metabolites in the Human Brain .1. Compartments and Water. Journal of Magnetic Resonance Series B, 102, 1-8.

FABER, C. \& WEBB, G. A. (2007) Resolution Enhancement in In Vivo NMR Spectroscopy. Annual Reports on NMR Spectroscopy. Academic Press.

FRAHM, J., BRUHN, H., GYNGELL, M. L., MERBOLDT, K. D., HANICKE, W. \& SAUTER, R. (1989a) Localized high-resolution proton NMR spectroscopy using stimulated echoes: initial applications to human brain in vivo. Magn Reson Med, 9, 79-93.

FRAHM, J. \& HÄNICKE, W. (2000) Single Voxel Localized Proton NMR Spectroscopy of Human Brain In Vivo. IN YOUNG, I. R. (Ed.) Methods in Biomedical Magnetic Resonance Imaging and Spectroscopy. New York, Wiley.

FRAHM, J., MERBOLDT, K. D. \& HANICKE, W. (1987) Localized Proton Spectroscopy Using Stimulated Echoes. Journal of Magnetic Resonance, 72, 502-508.

FRAHM, J., MICHAELIS, T., MERBOLDT, K. D., BRUHN, H., GYNGELL, M. L. \& HANICKE, W. (1990) Improvements in Localized Proton NMR Spectroscopy of Human Brain. Water Suppression, Short Echo Times, and $1 \mathrm{ml}$ Resolution. Journal of Magnetic Resonance, 90, 464-473.

FRAHM, J., MICHAELIS, T., MERBOLDT, K. D., HANICKE, W., GYNGELL, M. L., CHIEN, D. \& BRUHN, H. (1989b) Localized NMR spectroscopy in vivo. Progress and problems. NMR in Biomedicine, 2, 188-95.

FUJIMORI, H., MICHAELIS, T., WICK, M. \& FRAHM, J. (1998) Proton $T_{2}$ relaxation of cerebral metabolites during transient global ischemia in rat brain. Magnetic Resonance in Medicine, 39, 647-650.

GEURTS, J. J. G., BARKHOF, F., CASTELIJNS, J. A., UITDEHAAG, B. M. J., POLMAN, C. H. \& POUWELS, P. J. W. (2004) Quantitative ${ }^{1}$ H-MRS of healthy human cortex, hippocampus, and thalamus: Metabolite concentrations, quantification precision, and reproducibility. Journal of Magnetic Resonance Imaging, 20, 366-371.

GILLIES, R. J. \& MORSE, D. L. (2005) In vivo magnetic resonance spectroscopy in cancer. Annual Review of Biomedical Engineering, 7, 287-326.

GOVINDARAJU, V., YOUNG, K. \& MAUDSLEY, A. A. (2000) Proton NMR chemical shifts and coupling constants for brain metabolites. NMR Biomed, 13, 129-53.

GRUBER, S., MLYNÁRIK, V. \& MOSER, E. (2003) High-resolution 3D proton spectroscopic imaging of the human brain at $3 \mathrm{~T}$ : SNR issues and application for anatomy-matched voxel sizes. Magnetic Resonance in Medicine, 49, 299-306.

GRUETTER, R. (1993) Automatic, Localized Invivo Adjustment of All 1st-Order and 2nd-Order Shim Coils. Magnetic Resonance in Medicine, 29, 804-811.

GRUETTER, R. \& BOESCH, C. (1992) Fast, noniterative shimming of spatially localized signals. In vivo analysis of the magnetic field along axes. Journal of Magnetic Resonance (1969), 96, 323-334. 
GRUETTER, R. \& TKÁĆ, I. (2000) Field mapping without reference scan using asymmetric echoplanar techniques. Magnetic Resonance in Medicine, 43, 319-23.

GRUETTER, R., WEISDORF, S. A., RAJANAYAGAN, V., TERPSTRA, M., MERKLE, H., TRUWIT, C. L., GARWOOD, M., NYBERG, S. L. \& UGURBIL, K. (1998) Resolution Improvements in in Vivo ${ }^{1}$ H NMR Spectra with Increased Magnetic Field Strength. Journal of Magnetic Resonance, $135,260-264$.

GUÉRON, M. \& PLATEAU, P. (2007) Water Signal Suppression in NMR of Biomolecules, John Wiley \& Sons, Ltd.

GYNGELL, M. L., ELLERMANN, J., MICHAELIS, T., HANICKE, W., MERBOLDT, K. D., BRUHN, H. \& FRAHM, J. (1991a) Noninvasive H-1-Nmr Spectroscopy of the Rat-Brain Invivo Using a Short Echo Time Steam Localization Sequence. Nmr in Biomedicine, 4, 150-156.

GYNGELL, M. L., HOEHN-BERLAGE, M., KLOIBER, O., MICHAELIS, T., ERNESTU, R. I., HÖRSTERMANN, D. \& FRAHM, J. (1992) Localized proton NMR spectroscopy of experimental gliomas in rat brain In Vivo. Nmr in Biomedicine, 5, 335-340.

GYNGELL, M. L., MICHAELIS, T., HORSTERMANN, D., BRUHN, H., HANICKE, W., MERBOLDT, K. D. \& FRAHM, J. (1991b) Cerebral Glucose Is Detectable by Localized Proton NmrSpectroscopy in Normal Rat-Brain Invivo. Magnetic Resonance in Medicine, 19, 489-495.

HAASE, A., FRAHM, J., HANICKE, W. \& MATTHAEI, D. (1985) ${ }^{1}$ H NMR chemical shift selective (CHESS) imaging. Phys Med Biol, 30, 341-4.

HAHN, E. L. (1950) Spin Echoes. Physical Review, 80, 580-594.

HAMMEN, T., STADLBAUER, A., TOMANDL, B., GANSLANDT, O., PAULI, E., HUK, W., NEUNDÖRFER, B. \& STEFAN, H. (2005) Short TE single-voxel ${ }^{1} \mathrm{H}-\mathrm{MR}$ spectroscopy of hippocampal structures in healthy adults at 1.5 Tesla-how reproducible are the results? Nmr in Biomedicine, 18, 195-201.

HANCU, I., ZIMMERMAN, E. A., SAILASUTA, N. \& HURD, R. E. (2005) ${ }^{1}$ H MR spectroscopy using TE averaged PRESS: A more sensitive technique to detect neurodegeneration associated with Alzheimer's disease. Magnetic Resonance in Medicine, 53, 777-782.

HELMS, G. (2000) A precise and user-independent quantification technique for regional comparison of single volume proton MR spectroscopy of the human brain. $\mathrm{Nmr}$ in Biomedicine, 13, 398-406.

HELMS, G. (2008) The principles of quantification applied to in vivo proton MR spectroscopy. Eur J Radiol, 67, 218-29.

HENNIG, J., NAUERTH, A. \& FRIEDBURG, H. (1986) RARE imaging: A fast imaging method for clinical MR. Magnetic Resonance in Medicine, 3, 823-833.

HENNIG, J., PFISTER, H., ERNST, T. \& OTT, D. (1992) Direct absolute quantification of metabolites in the human brain with in vivo localized proton spectroscopy. Nmr in Biomedicine, 5, 193-199.

HOFMANN, L., SLOTBOOM, J., BOESCH, C. \& KREIS, R. (1999) Model fitting of ${ }^{1} \mathrm{H}-\mathrm{MR}$ spectra of the human brain: incorporation of short- $T_{1}$ components and evaluation of parameterized vs. non-parameterized models. 7th Meeting of ISMRM. Philadelphia.

HOFMANN, L., SLOTBOOM, J., JUNG, B., MALOCA, P., BOESCH, C. \& KREIS, R. (2002) Quantitative ${ }^{1} \mathrm{H}$-magnetic resonance spectroscopy of human brain: Influence of composition and parameterization of the basis set in linear combination model-fitting. Magnetic Resonance in Medicine, 48, 440-53.

HONG, S.-T., BALLA, D. Z., CHOI, C. \& POHMANN, R. (2011a) Rat strain-dependent variations in brain metabolites detected by in vivo ${ }^{1} \mathrm{H}$ NMR spectroscopy at $16.4 \mathrm{~T}$. Nmr in Biomedicine, 24, 1401-1407.

HONG, S.-T., BALLA, D. Z. \& POHMANN, R. (2011b) Determination of regional variations and reproducibility in in vivo ${ }^{1} \mathrm{H}$ NMR spectroscopy of the rat brain at $16.4 \mathrm{~T}$. Magnetic Resonance in Medicine, 66, 11-17. 
HONG, S.-T., BALLA, D. Z., SHAJAN, G., CHOI, C., UĞURBIL, K. \& POHMANN, R. (2011c) Enhanced neurochemical profile of the rat brain using in vivo ${ }^{1} \mathrm{H}$ NMR spectroscopy at $16.4 \mathrm{~T}$. Magnetic Resonance in Medicine, 65, 28-34.

HORE, P. (1983) Solvent suppression in Fourier transform nuclear magnetic resonance. Journal of Magnetic Resonance, 55, 283-300.

HORSKÁ, A., CALHOUN, V., BRADSHAW, D. \& BARKER, P. (2002) Rapid method for correction of CSF partial volume in quantitative proton MR spectroscopic imaging. Magnetic Resonance in Medicine, 48, 555-558.

HOWE, F. A., MAXWELL, R. J., SAUNDERS, D. E., BROWN, M. M. \& GRIFFITHS, J. R. (1993) Proton Spectroscopy In-Vivo. Magnetic Resonance Quarterly, 9, 31-59.

IN 'T ZANDT, H. J. A., VAN DER GRAAF, M. \& HEERSCHAP, A. (2001) Common processing of in vivo MR spectra. Nmr in Biomedicine, 14, 224-232.

INGLESE, M., SPINDLER, M., BABB, J. S., SUNENSHINE, P., LAW, M. \& GONEN, O. (2006) Field, Coil, and Echo-Time Influence on Sensitivity and Reproducibility of Brain Proton MR Spectroscopy. American Journal of Neuroradiology, 27, 684-688.

JANSEN, J. F. A., BACKES, W. H., NICOLAY, K. \& KOOI, M. E. (2006) ${ }^{1} \mathrm{H}$ MR Spectroscopy of the Brain: Absolute Quantification of Metabolites. Radiology, 240, 318-332.

KEEVIL, S. F. (2006) Spatial localization in nuclear magnetic resonance spectroscopy Physics in Medicine \& Biology, 51, R579-636.

KLOSE, U. (1990) In vivo proton spectroscopy in presence of eddy currents. Magnetic Resonance in Medicine, 14, 26-30.

$\mathrm{KOCH}, \mathrm{K}$. M., ROTHMAN, D. L. \& DE GRAAF, R. A. (2009) Optimization of static magnetic field homogeneity in the human and animal brain in vivo. Progress in Nuclear Magnetic Resonance Spectroscopy, 54, 69-96.

KREIS, R. (1997) Quantitative localized ${ }^{1} \mathrm{H}$ MR spectroscopy for clinical use. Progress in Nuclear Magnetic Resonance Spectroscopy, 31, 155-195.

KREIS, R. (2004) Issues of spectral quality in clinical ${ }^{1} \mathrm{H}$-magnetic resonance spectroscopy and a gallery of artifacts. Nmr in Biomedicine, 17, 361-381.

KREIS, R. \& BOESCH, C. (2003) Bad Spectra Can Be Better Than Good Spectra. 11th Meeting of ISMRM. Toronto.

KREIS, R., ERNST, T. \& ROSS, B. D. (1993a) Absolute Quantitation of Water and Metabolites in the Human Brain .2. Metabolite Concentrations. Journal of Magnetic Resonance Series B, 102, 9-19.

KREIS, R., ERNST, T. \& ROSS, B. D. (1993b) Development of the human brain: in vivo quantification of metabolite and water content with proton magnetic resonance spectroscopy. Magn Reson Med, 30, 424-37.

KREIS, R., SLOTBOOM, J., HOFMANN, L. \& BOESCH, C. (2005) Integrated data acquisition and processing to determine metabolite contents, relaxation times, and macromolecule baseline in single examinations of individual subjects. Magnetic Resonance in Medicine, 54, 761-768.

LANGE, T., DYDAK, U., ROBERTS, T. P. L., ROWLEY, H. A., BJELJAC, M. \& BOESIGER, P. (2006) Pitfalls in Lactate Measurements at 3T. American Journal of Neuroradiology, 27, 895901.

LAUTERBUR, P. C. (1973) Image Formation by Induced Local Interactions: Examples Employing Nuclear Magnetic Resonance. Nature, 242, 190-191.

LEI, H., POITRY-YAMATE, C., PREITNER, F., THORENS, B. \& GRUETTER, R. (2010) Neurochemical profile of the mouse hypothalamus using in vivo1H MRS at 14.1T. Nmr in Biomedicine, 23, 578-583.

LIEBETANZ, D., FAUSER, S., MICHAELIS, T., CZEH, B., WATANABE, T., PAULUS, W., FRAHM, J. \& FUCHS, E. (2003) Safety aspects of chronic low-frequency transcranial magnetic 
stimulation based on localized proton magnetic resonance spectroscopy and histology of the rat brain. Journal of Psychiatric Research, 37, 277-286.

MACRì, M. A., GARREFFA, G., GIOVE, F., GUARDATI, M., AMBROSINI, A., COLONNESE, C. \& MARAVIGLIA, B. (2004) In vivo quantitative ${ }^{1} \mathrm{H}$ MRS of cerebellum and evaluation of quantitation reproducibility by simulation of different levels of noise and spectral resolution. Magnetic Resonance Imaging, 22, 1385-1393.

MANSFIELD, P. \& GRANNELL, P. K. (1973) NMR 'diffraction' in solids? Journal of Physics C: Solid State Physics, 6, L422.

MICHAELIS, T., BORETIUS, S. \& FRAHM, J. (2009) Localized proton MRS of animal brain in vivo: Models of human disorders. Progress in Nuclear Magnetic Resonance Spectroscopy, In Press, Corrected Proof.

MICHAELIS, T., DE BIURRUN, G., WATANABE, T., FRAHM, J., OHL, F. \& FUCHS, E. (2001) Genderspecific alterations of cerebral metabolites with aging and cortisol treatment. Journal of Psychiatric Research, 35, 231-237.

MICHAELIS, T. \& FRAHM, J. (2005) Low-field vs. high-field proton MRS of mouse brain in vivo. Magnetic Resonance in Medicine, 54, 1046-1047.

MICHAELIS, T., HELMS, G., MERBOLDT, K. D., HANICKE, W., BRUHN, H. \& FRAHM, J. (1993a) Identification of Scyllo-Inositol in Proton Nmr-Spectra of Human Brain Invivo. Nmr in Biomedicine, 6, 105-109.

MICHAELIS, T., MERBOLDT, K. D., BRUHN, H., HANICKE, W. \& FRAHM, J. (1993b) Absolute Concentrations of Metabolites in the Adult Human Brain In vivo - Quantification of Localized Proton Mr Spectra. Radiology, 187, 219-227.

MICHAELIS, T., MERBOLDT, K. D., HANICKE, W., GYNGELL, M. L., BRUHN, H. \& FRAHM, J. (1991) On the Identification of Cerebral Metabolites in Localized ${ }^{1} \mathrm{H}$ Nmr Spectra of Human Brain In vivo. Nmr in Biomedicine, 4, 90-98.

MICHAELIS, T., WICK, M., FUJIMORI, H., MATSUMURA, A. \& FRAHM, J. (1999) Proton MRS of oral creatine supplementation in rats. Cerebral metabolite concentrations and ischemic challenge. Nmr in Biomedicine, 12, 309-314.

MILNE, A., MACQUEEN, G. M., YUCEL, K., SORENI, N. \& HALL, G. B. C. (2009) Hippocampal metabolic abnormalities at first onset and with recurrent episodes of a major depressive disorder: A proton magnetic resonance spectroscopy study. Neuroimage, 47, 36-41.

MOONEN, C. T. W., SOBERING, G., VAN ZIJL, P. C. M., GILLEN, J., VON KIENLIN, M. \& BIZZI, A. (1992) Proton spectroscopic imaging of human brain. Journal of Magnetic Resonance (1969), 98, 556-575.

MOONEN, C. T. W. \& VANZIJL, P. C. M. (1990) Highly effective water suppression for in vivo proton NMR spectroscopy (DRYSTEAM). Journal of Magnetic Resonance, 88, 28-41.

MORRIS, G. \& FREEMAN, R. (1978) Selective excitation in Fourier transform nuclear magnetic resonance. Journal of Magnetic Resonance (1969), 29, 433-462.

NAGAE-POETSCHER, L. M., BONEKAMP, D., BARKER, P. B., BRANT, L. J., KAUFMANN, W. E. \& HORSKÁ, A. (2004) Asymmetry and gender effect in functionally lateralized cortical regions: A proton MRS imaging study. Journal of Magnetic Resonance Imaging, 19, 2733.

NATT, O., BEZKOROVAYNYY, V., MICHAELIS, T. \& FRAHM, J. (2005) Use of phased array coils for a determination of absolute metabolite concentrations. Magnetic Resonance in Medicine, $53,3-8$.

OBERG, J., SPENGER, C., WANG, F.-H., ANDERSSON, A., WESTMAN, E., SKOGLUND, P., SUNNEMARK, D., NORINDER, U., KLASON, T., WAHLUND, L.-O. \& LINDBERG, M. (2008) Age related changes in brain metabolites observed by $1 \mathrm{H}$ MRS in APP/PS1 mice. Neurobiology of Aging, 29, 1423-1433. 
OGG, R. J., KINGSLEY, P. B. \& TAYLOR, J. S. (1994) WET, a $T_{1^{-}}$and $B_{1}$-insensitive watersuppression method for in vivo localized ${ }^{1} \mathrm{H}$ NMR spectroscopy. J Magn Reson B, 104, 110.

ORDIDGE, R. J., CONNELLY, A. \& LOHMAN, J. A. B. (1986) Image-selected in Vivo spectroscopy (ISIS). A new technique for spatially selective nmr spectroscopy. Journal of Magnetic Resonance (1969), 66, 283-294.

ÖZ, G., NELSON, C. D., KOSKI, D. M., HENRY, P. G., MARJANSKA, M., DEELCHAND, D. K., SHANLEY, R., EBERLY, L. E., ORR, H. T. \& CLARK, H. B. (2010) Noninvasive Detection of Presymptomatic and Progressive Neurodegeneration in a Mouse Model of Spinocerebellar Ataxia Type 1. Journal of Neuroscience, 30, 3831.

PATT, S. \& SYKES, B. (1972) Water eliminated Fourier transform NMR spectroscopy. Journal of Chemical Physics, 56, 3182-3184.

PFEUfFER, J., JUCHEM, C., MERKLE, H., NAUERTH, A. \& LOGOTHETIS, N. K. (2004) High-field localized ${ }^{1} \mathrm{H}$ NMR spectroscopy in the anesthetized and in the awake monkey. Magnetic Resonance Imaging, 22, 1361-1372.

PFEUfFER, J., TKÁC, I., PROVENCHER, S. W. \& GRUETTER, R. (1999) Toward an in Vivo Neurochemical Profile: Quantification of 18 Metabolites in Short-Echo-Time ${ }^{1} \mathrm{H}$ NMR Spectra of the Rat Brain. Journal of Magnetic Resonance, 141, 104-120.

PIOTTO, M., SAUDEK, V. \& SKLENÁ , V. (1992) Gradient-tailored excitation for single-quantum NMR spectroscopy of aqueous solutions. Journal of biomolecular NMR, 2, 661-665.

POHMANN, R. (2011) Physical Basics of NMR.

POSSE, S., SCHUKNECHT, B., SMITH, M. E., VANZIJL, P. C. M., HERSCHKOWITZ, N. \& MOONEN, C. T. W. (1993) Short echo time proton MR spectroscopic imaging. Journal of Computer Assisted Tomography, 17, 1-14.

POULLET, J.-B., SIMA, D. M. \& VAN HUFFEL, S. (2008) MRS signal quantitation: A review of timeand frequency-domain methods. Journal of Magnetic Resonance, 195, 134-144.

POUWELS, P. J. W. \& FRAHM, J. (1998) Regional metabolite concentrations in human brain as determined by quantitative localized proton MRS. Magnetic Resonance in Medicine, 39, 53-60.

PROCTOR, W. G. \& YU, F. C. (1950) The Dependence of a Nuclear Magnetic Resonance Frequency upon Chemical Compound. Physical Review, 77, 717-717.

PROVENCHER, S. W. (1993) Estimation of metabolite concentrations from localized in vivo proton NMR spectra. Magnetic Resonance in Medicine, 30, 672-9.

PROVENCHER, S. W. (2001) Automatic quantitation of localized in vivo H-1 spectra with LCModel. Nmr in Biomedicine, 14, 260-264.

PURCELL, E. M., TORREY, H. C. \& POUND, R. V. (1946) Resonance Absorption by Nuclear Magnetic Moments in a Solid. Physical Review, 69, 37-38.

RAMSEY, N. F. \& PURCELL, E. M. (1952) Interactions between Nuclear Spins in Molecules. Physical Review, 85, 143-144.

RENEMA, W. K. J., SCHMIDT, A., VAN ASTEN, J. J. A., OERLEMANS, F., ULLRICH, K., WIERINGA, B., ISBRANDT, D. \& HEERSCHAP, A. (2003) MR spectroscopy of muscle and brain in guanidinoacetate methyltransferase (GAMT)-deficient mice: Validation of an animal model to study creatine deficiency. Magnetic Resonance in Medicine, 50, 936-943.

RIETH, K. G., FUJIWARA, K., DI CHIRO, G., KLATZO, I., BROOKS, R. A., JOHNSTON, G. S., O'CONNOR, C. M. \& MITCHELL, L. G. (1980) Serial measurements of CT attenuation and specific gravity in experimental cerebral edema. Radiology, 135, 343-348.

SCHIRMER, T. \& AUER, D. P. (2000) On the reliability of quantitative clinical magnetic resonance spectroscopy of the human brain. NMR in Biomedicine, 13, 28-36.

SCHUFF, N., EZEKIEL, F., GAMST, A. C., AMEND, D. L., CAPIZZANO, A. A., MAUDSLEY, A. A. \& WEINER, M. (2001) Region and tissue differences of metabolites in normally aged brain 
using multislice ${ }^{1} \mathrm{H}$ magnetic resonance spectroscopic imaging. Magnetic Resonance in Medicine, 45, 899-907.

SCHWARCZ, A., BERENTE, Z., OSZ, E. \& DOCZI, T. (2001) In vivo water quantification in mouse brain at 9.4 Tesla in a vasogenic edema model. Magnetic Resonance in Medicine, 46, 1246-1249.

SCHWARCZ, A., NATT, O., WATANABE, T., BORETIUS, S., FRAHM, J. \& MICHAELIS, T. (2003) Localized proton MRS of cerebral metabolite profiles in different mouse strains. Magnetic Resonance in Medicine, 49, 822-7.

SEAQUIST, E. R. \& GRUETTER, R. (1998) Identification of a high concentration of scyllo-inositol in the brain of a healthy human subject using ${ }^{1} \mathrm{H}$ - and ${ }^{13} \mathrm{C}-\mathrm{NMR}$. Magnetic Resonance in Medicine, 39, 313-316.

SEEGER, U., KLOSE, U., MADER, I., GRODD, W. \& NÄGELE, T. (2003) Parameterized evaluation of macromolecules and lipids in proton MR spectroscopy of brain diseases. Magnetic Resonance in Medicine, 49, 19-28.

SHOUP, R. R., BECKER, E. D. \& FARRAR, T. C. (1972) The driven equilibrium fourier transform NMR technique: An experimental study. Journal of Magnetic Resonance (1969), 8, 298310.

SHUNGU, D. \& GLICKSON, J. (1993) Sensitivity and localization enhancement in multinuclear in vivo NMR spectroscopy by outer volume presaturation. Magnetic Resonance in Medicine, 30, 661-661.

SKLENAR, V., PIOTTO, M., LEPPIK, R. \& SAUDEK, V. (1993) Gradient-tailored water suppression for $1 \mathrm{H}-15 \mathrm{~N}$ HSQC experiments optimized to retain full sensitivity. Journal of magnetic resonance. Series $A$ (Print), 102, 241-245.

SOHER, B. J., HURD, R. E., SAILASUTA, N. \& BARKER, P. B. (1996) Quantitation of automated single-voxel proton MRS using cerebral water as an internal reference. Magnetic Resonance in Medicine, 36, 335-339.

STANLEY, J. A., DROST, D. J., WILLIAMSON, P. C. \& THOMPSON, R. T. (1995) The use of a priori knowledge to quantify short echo in vivo ${ }^{1} \mathrm{H}$ MR Spectra. Magnetic Resonance in Medicine, 34, 17-24.

STOREY, P. (2005) Introduction to Magnetic Resonance Imaging and Spectroscopy. IN PRASAD, P. V. (Ed.) Magnetic Resonance Imaging: Methods and Biologic Applications.

TAKAGI, H., SHAPIRO, K., MARMAROU, A. \& WISOFF, H. (1981) Microgravimetric analysis of human brain tissue Correlation with computerized tomography scanning. Journal of Neurosurgery, 54, 797-801.

THOMPSON, R. B. \& ALLEN, P. S. (1998) A new multiple quantum filter design procedure for use on strongly coupled spin systems found in vivo: Its application to glutamate. Magnetic Resonance in Medicine, 39, 762-771.

THOMPSON, R. B. \& ALLEN, P. S. (2001) Response of metabolites with coupled spins to the STEAM sequence. Magnetic Resonance in Medicine, 45, 955-965.

TKÁĆ, I. \& GRUETTER, R. (2005) In vivo ${ }^{1}$ H NMR spectroscopy and neurochemistry. Quantification matters. Magnetic Resonance in Medicine, 54, 1048-1049.

TKÁĆ, I., HENRY, P. G., ANDERSEN, P., KEENE, C. D., LOW, W. C. \& GRUETTER, R. (2004) Highly resolved in vivo ${ }^{1} \mathrm{H}$ NMR spectroscopy of the mouse brain at $9.4 \mathrm{~T}$. Magnetic Resonance in Medicine, 52, 478-484.

TKÁĆ, I., OZ, G., ADRIANY, G., UGURBIL, K. \& GRUETTER, R. (2009) In Vivo ${ }^{1}$ H NMR Spectroscopy of the Human Brain at High Magnetic Fields: Metabolite Quantification at 4T vs. 7T. Magnetic Resonance in Medicine, 62, 868-879.

TKÁĆ, I., RAO, R., GEORGIEFF, M. K. \& GRUETTER, R. (2003) Developmental and regional changes in the neurochemical profile of the rat brain determined by in vivo ${ }^{1} \mathrm{H} N M R$ spectroscopy. Magnetic Resonance in Medicine, 50, 24-32. 
TKÁĆ, I., STARCUK, Z., CHOI, I. Y. \& GRUETTER, R. (1999) In vivo ${ }^{1}$ H NMR spectroscopy of rat brain at $1 \mathrm{~ms}$ echo time. Magnetic Resonance in Medicine, 41, 649-656.

TKÁĆ, I., UGURBIL, K. \& GRUETTER, R. (2002) On the quantification of low concentration metabolites by ${ }^{1} \mathrm{H}$ NMR spectroscopy in the human brain at 7 Tesla. 10th Meeting of ISMRM. Honolulu.

TORACK, R. M., ALCALA, H., GADO, M. \& BURTON, R. (1976) Correlative assay of computerized cranial tomography $\mathrm{CCT}$, water content and specific gravity in normal and pathological postmortem brain. Journal of Neuropathology and Experimental Neurology, 35, 385-392.

TRABESINGER, A. H., MEIER, D., DYDAK, U., LAMERICHS, R. \& BOESIGER, P. (2005) Optimizing PRESS localized citrate detection at 3 Tesla. Magnetic Resonance in Medicine, 54, 51-58.

VAN DER HART, M. G. C., CZEH, B., DE BIURRUN, G., MICHAELIS, T., WATANABE, T., NATT, O., ASHERY-PADAN, R., FRAHM, J. \& FUCHS, E. (2002) Substance $P$ receptor antagonist and clomipramine prevent stress-induced alterations in cerebral metabolites, cytogenesis in the dentate gyrus and hippocampal volume, Basingstoke, ROYAUME-UNI, Nature Publishing Group.

WEBB, P., SAILASUTA, N., KOHLER, S., RAIDY, T., MOATS, R. \& HURD, R. (1994) Automated singlevoxel proton MRS: technical development and multisite verification. Magnetic Resonance in Medicine, 31, 365-373.

WICK, M., FUJIMORI, H., MICHAELIS, T. \& FRAHM, J. (1999) Brain water diffusion in normal and creatine-supplemented rats during transient global ischemia. Magnetic Resonance in Medicine, 42, 798-802.

XIN, L., GAMBAROTA, G., MLYNÁRIK, V. \& GRUETTER, R. (2008) Proton $T_{2}$ relaxation time of Jcoupled cerebral metabolites in rat brain at 9.4 T. Nmr in Biomedicine, 21, 396-401. 


\section{Curriculum Vitae}

\section{Personal Information}

Name:

Alireza Abaei Tafresh

Date of Birth:

Nationality:

9 September 1975

Iranian

\section{Studies}

Since 2007

PhD Student

1998 to 2001

Georg-August-University of Göttingen

Studies of Nuclear Engineering- Application in Medicine Master of Science Degree

at Amir Kabir University of Technology (Tehran Polytechnic)

1993 to 1998 Studies of Physics (Nuclear Physics)

Bachelor of Science Degree

at Shahid Beheshti University, Tehran

\section{Professional Experience}

2001 to 2006

MR Engineer

Philips Medical Systems Iran Authorized Distributor,

Tehran, Iran 


\section{List of Publications}

Thomas Michaelis, Alireza Abaei, Susann Boretius, Roland Tammer, Jens Frahm, Christina Schlumbohm, Eberhard Fuchs. Intrauterine hyperexposure to dexamethasone of the common marmoset monkey revealed normal cerebral metabolite concentrations in adulthood as assessed by quantitative proton magnetic resonance spectroscopy in vivo. Journal of Medical Primatology, 38 (3): 213-218 (2009)

T. Michaelis, A. Abaei, R. Tammer, S. Boretius, C. Schlumbohm, E. Fuchs, and J. Frahm. Cerebral Metabolism of Adult Marmoset Monkeys After Intrauterine Hyperexposure to Dexamethasone. Proceeding International Society for Magnetic Resonance in Medicine (ISMRM), 2007.

A. Abaei, S. Boretius, R. Tammer, J. Frahm, T. Michaelis. On the relative detectability of strongly coupled metabolite resonances in localized proton MR spectra at low and high field strength. Proceeding European Society for Magnetic Resonance in Medicine and Biology (ESMRMB), 2008

\section{Other Publications}

Alireza Abaei. High-field proton MRS of mouse brain metabolites, MPIbpc News (Sep. 2009): Doktorandenseminar, Max-Planck-Institut für biophysikalische Chemie, Vol. 15, issue 9, page 30 . 